\title{
Perception of the body in space: mechanisms
}

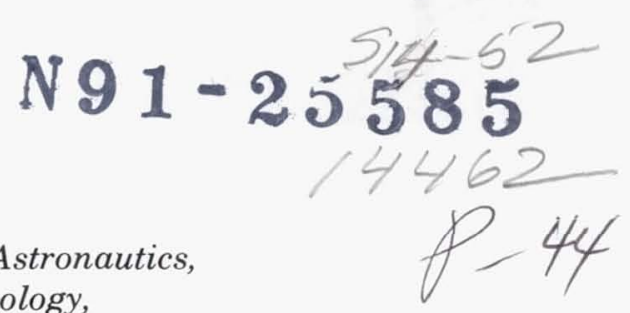

\author{
LA URENCER. Y O U N G
}

Department of Aeronautics and Astronautics, Massachusetts Institute of Technology, Cambridge, Massachusetts

\section{CHAPTER CONTENTS}

Perception of Orientation Based on Multiple Sensory Modalities Semicircular canals

Otolith organs

Somatosensory cues

Limb position

Psychophysical Measures of Perception of Orientation and Motion

Angular Acceleration

Rotation in dark

Active versus passive movement

Torsion-pendulum model

Thresholds

Oculogyral illusion

Adaptation

Caloric and alcohol effects

Linear Motion and Gravity

Nature of linear accelerometers

Static orientation to vertical

Contributions of nonlabyrinthine sensors to perception of tilt

Reduced somatosensory cues

Labyrinthine defective subjects

Amplified somatosensory or postural cues

Dynamic Response of Otolith System

Ambiguity of subjective response to acceleration

Linear acceleration steps

Sinusoidal linear acceleration

Combined Rotational and Linear Accelerations

Consistent and inconsistent vestibular signals

Centripetal acceleration

Cross-coupled angular acceleration; Coriolis illusion

Rotation about off-vertical axes

Visual Effects on Perceived Orientation-Models

Static visual orientation

Moving visual fields

Static visual-vestibular interaction

Vection and dynamic visual-vestibular interaction

Spatial Orientation in Altered Environments

Motion sickness

Summary

THE PRINCIPAL TOPIC TREATED IN THIS CHAPTER is the perception of body orientation and motion in space and the extent to which these perceptual abstractions can be related directly to our knowledge of sensory mechanisms, particularly for the vestibular apparatus.

The relationship between physical variables trans- duced by the sensory systems and human perception of orientation was still a subject of vigorous debate during the last century. The notion that perception could be discussed in terms of a machine acting on external variables was foreign to the basic ideas of Kant, which influenced nineteenth-century thinkers so much. The central role of the vestibular apparatus as the organ of equilibrium had not always been accepted, even after the demonstration of their importance in postural control (55). The long and unfortunate distinction between physical stimuli and sensory processes on the one hand and perception on the other hand has been reviewed by Boring (16) and by Teuber (152) and this distinction is placed in its historical perspective in the chapter by Jung in this Handbook.

Increasingly, sensory end-organ characteristics and central nervous system activity in response to complex multisensory stimuli are related to the perception of body movement reported by a human being under similar stimulus conditions. It is clearly appropriate to relate sensory-unit activity resulting from body-motion stimuli to such purposeful motor activities as head or eye stabilization. It is no less relevant to relate underlying sensory transduction and the higher processing of these afferent signals to the generation of motion perception. Long after it was fully recognized that we see with our eyes and hear with our ears, the origin of the perception of spatial orientation remained a mystery. At the beginning of the nineteenth century the sense of equilibrium was still judged to be related to shifting the fluid in the cranium as the head orientation was changed by gravity. Perhaps the most critical experiment in creating a sensory basis for spatial orientation was performed by Flourens (55). He demonstrated the essential role of the semicircular canals in postural stability and equilibrium and, incidentally, related their stimulation to the genesis of motion sickness. It remained for Mach, a physicist and natural scientist of the mid-nineteenth century, however, to relate the physical characteristics of the semicircular canals and the otolith system to the quantitative perceptual measures of tilt and rotation. In his 
influential treatise, Mach (106) found it necessary to explain at length why motion sensation had to be associated with mechanical stimuli of the sensory organs. Remnants of this unfortunate dichotomy between stimulus and perception remain to the present time.

The next major thrust in the causal link between vestibular organ stimulation and perception of orientation came through demonstrations that involuntary eye movements, as a special case of sensorimotor reactions, were also driven directly by vestibular stimulation. The recognition that slow compensatory eye movements would result from direct cupula stimulation of semicircular canals (48) and later that ocular counterrolling, or compensatory torsion of the eye about the axis of gaze, could be attributed to otolith as well as semicircular canal stimulation greatly enhanced the ability of workers in the field of human spatial orientation to relate vestibular stimulation to perception and eye movements.

The first half of the twentieth century saw a growth of research activity devoted toward quantitative evaluation of the physical transduction properties of the vestibular organs, especially of the semicircular canals, based largely on measurements of human perception of rotation and tilt during deterministic transient motions or steady-state sinusoidal oscillations. The physical characteristics of the semicircular canals, clarified by the demonstrations of Steinhausen (147) and Dohlman (47) that the cupula does not normally allow endolymph to flow freely through the ampulla, were translated into differential equation form $(148,160)$. Recently it has been possible to make quantitative estimates (129) and actual measurements $(88,127)$ of cupula deflection and to relate them to rotation sensation for arbitrary rotation stimuli.

It is tempting but misleading to regard spatial orientation and postural reactions to environmental disturbances as automatic or reflex reactions. Certainly, many environmental disturbances lead to highly stereotyped postural reactions that direct an animal toward a desirable situation, away from danger, or toward the reestablishment of a stable posture. There was a period following the turn of the century, based on the theories of Loeb (104), when such orienting behavior was described as thoroughly mechanistic. Orientation, at least in the lower animal, was conceived of as being totally determined by, for example, the presence of light or the angle relative to gravity. Postural reactions and locomotion were seen as reflexes totally independent of sensation. Even for invertebrates this extreme view of mechanistic orientation behavior has been largely discarded (58). Such automatic, machinelike postural orientation reactions are seen in mammals only under special circumstances. Specifically, on decerebrating a cat the automatic righting reactions are easily seen. It is tempting to attribute orientation sensations for the intact animal to direct pathways from graviceptor stimulation or asymmetric tactile stimulation. The actual perception of orientation, although relying on the various sensory inputs that also produce postural reactions, is heavily influenced by a number of cortical functions, including expectation of the input and habituation to a particular stimulus sequence.

The latter third of the twentieth century has seen a rebirth of interest in nonvestibular contributions to self-motion perception, especially the visually induced perception of motion occurring in response to a uniform moving field (86). These visual illusions of motion sensation, as well as the less-documented illusions of self-motion based on manipulation of tactile, proprioceptive, and auditory cues, lead to the current "systems view" of mechanisms of perception of the body in space. Under most circumstances the multiplicity of sensory inputs related to spatial orientation is processed by the central nervous system to produce only a single, usually nonambiguous, perception of body orientation and movement. The process by which these various sensory signals are reduced and compared with one another is known as multisensory integration. For example, the extent to which a given visual motion contributes to the perception of selfmotion is influenced strongly by the concurrent vestibular stimulation or lack of it. Furthermore, all of the multisensory integration logic is plastic or modifiable. Perceptual, postural, and eye-movement responses to identical sensory patterns exhibit habituation to repeated stimuli and adaptation to rearranged sensory signals. Thus, for example, the normal responses of rotation perception, compensatory eye movements, and postural reactions to combinations of head movement and visual-field rotation are all greatly modified by sensory stimulus rearrangements as simple as wearing reversing prisms $(63,64,125)$ or as complex as exposure to weightlessness. Furthermore, the systems view of this multisensory integration allows for the processing of sensory signals to be modified on the basis of the "expected response." This expected response might reflect either an efferent copy of active movement undertaken by the subject, which would normally produce a given feedback pattern, or it might reflect the expected continuation of a welllearned pattern of movement such as oscillation on a swing. This multisensory integration approach requires knowledge of the dynamic response of the various sensory organs associated with human spatial orientation as well as estimation of the physical situation in which the subject finds himself $(14,15)$.

\section{PERCEPTION OF ORIENTATION BASED ON MULTIPLE SENSORY MODALITIES}

A frequent problem in relating physical stimuli to perception is that the number of dimensions of the perception exceeds that of the stimuli. Teuber (152) pointed out that for audition, independent variations 
of only frequency and intensity lead to variations in the perception of pitch, density, volume, and loudness. For motion sensation, however, the situation is quite simple: perceptions are limited to linear and angular position and body motion in three dimensions. Referring to the standard definition of body axes in Figure 1 , the vector of orientation sensation includes tilt orientation with respect to the vertical (pitch and roll), angular heading orientation (yaw), their first derivatives (rotation rates), and possibly angular accelerations. Linear displacement (forward-backward, leftright, up-down) is a three-dimensional vector perceived as linear position; linear velocity and possibly linear acceleration are also sensed. Perceived spatial orientation is closely related to the actual linear and angular motions of the body, and many of the important differences between perception and true motion are explainable on the basis of the dynamic characteristics of the sensors. In marked contrast to studies of perception of sound or light, however, motion perception is usually based on simultaneous stimulation of one or more of several sensory systems. Visual, tactile, and proprioceptive stimuli, as well as vestibular inputs, can produce motion sensations by themselves and modify motion sensations in conjunction with other sensors. We do not distinguish among a visual motion sensation, a vestibular motion sensation, and a tactile motion sensation. Consequently, in discussing the mechanisms involved in motion perception it is not surprising that much of the emphasis is placed on the interaction among different sensory modalities rather than simply on the transfer characteristics from stimulus to sensation through a single sensory channel.

Motion perception is strongly influenced by mental set or the preconceived idea of the subject as to what types of motion are permissible or what the limits of the test apparatus may be. Active control by a subject

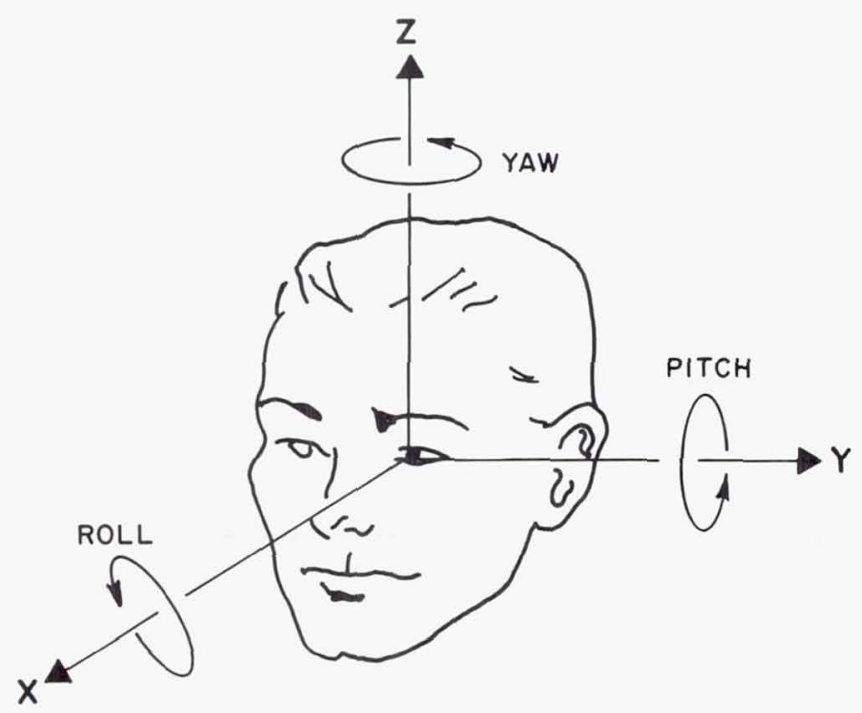

FIG. 1. Definition of axes for linear and angular motion. [From Hixon et al. (89).] of his own body motion in space, whether through direct muscular activity as in postural control and locomotion or through command of a vehicle, may result in vastly different perceptions of motion than would be true for a passive observer receiving the identical sensory stimuli. Habituation to repeated presentation of the same sensory stimuli may result in a decreased perception of body motion.

Simultaneous processing of information from the vestibular, visual, proprioceptive, and auditory channels regarding human spatial orientation is illustrated schematically in Figure 2. The spatial orientation sense cannot be turned off by closing one's eyes or by eliminating acoustic stimuli. The absence of any head movements to stimulate the vestibular system is not sensory deafferentation but rather a definite signal indicating the continuation of constant-velocity motion (including zero velocity). Proprioceptive and tactile inputs can never be removed except during periods of free fall, considered in more detail as a special case of environmental adaptation in section SPATIAL ORIENTATION IN ALTERED ENVIRONMENTS, p. 1060. The perception of relationships between parts of the body, such as the estimation of limb position, is not discussed in this chapter except as related to the interpretation of visual or vestibular signals. The important influence of active motor control on both actual and perceived motion of the body is indicated in Figure 2. Furthermore, the expected patterns of motion, whether based on continuation of an existing pattern or on recollection of the motion from the previous exposure to the same situation, are of vital importance in determining the manner in which multiple sensory inputs are combined to yield a single perception of spatial orientation and movement. Special cases of ambiguous stimuli resulting in confusion about spatial orientation or of behavior that alternates between two or more different orientations may result in vertigo or motion sickness and are discussed separately in Motion Sickness, p. 1061.

\section{Semicircular Canals}

The semicircular canals, as described in detail in the chapter by Goldberg and Fernández in this Handbook, are fluid-filled rings that respond to angular accelerations having a component normal to the plane of the ring by deviation of the cupula and stimulation of the hair cells in the crista. As a result of their arrangement in three roughly orthogonal planes within each labyrinth, the semicircular canals are able to detect and transduce angular accelerations about any axis in space. Because of their narrow lumen and the large contribution of viscous force inside the tube relative to inertial forces, they act as approximate integrators. Consequently for all but very low frequencies of stimulation their output reflects angular velocity rather than angular acceleration of the head with respect to inertial space. They fail as angular-velocity trans- 


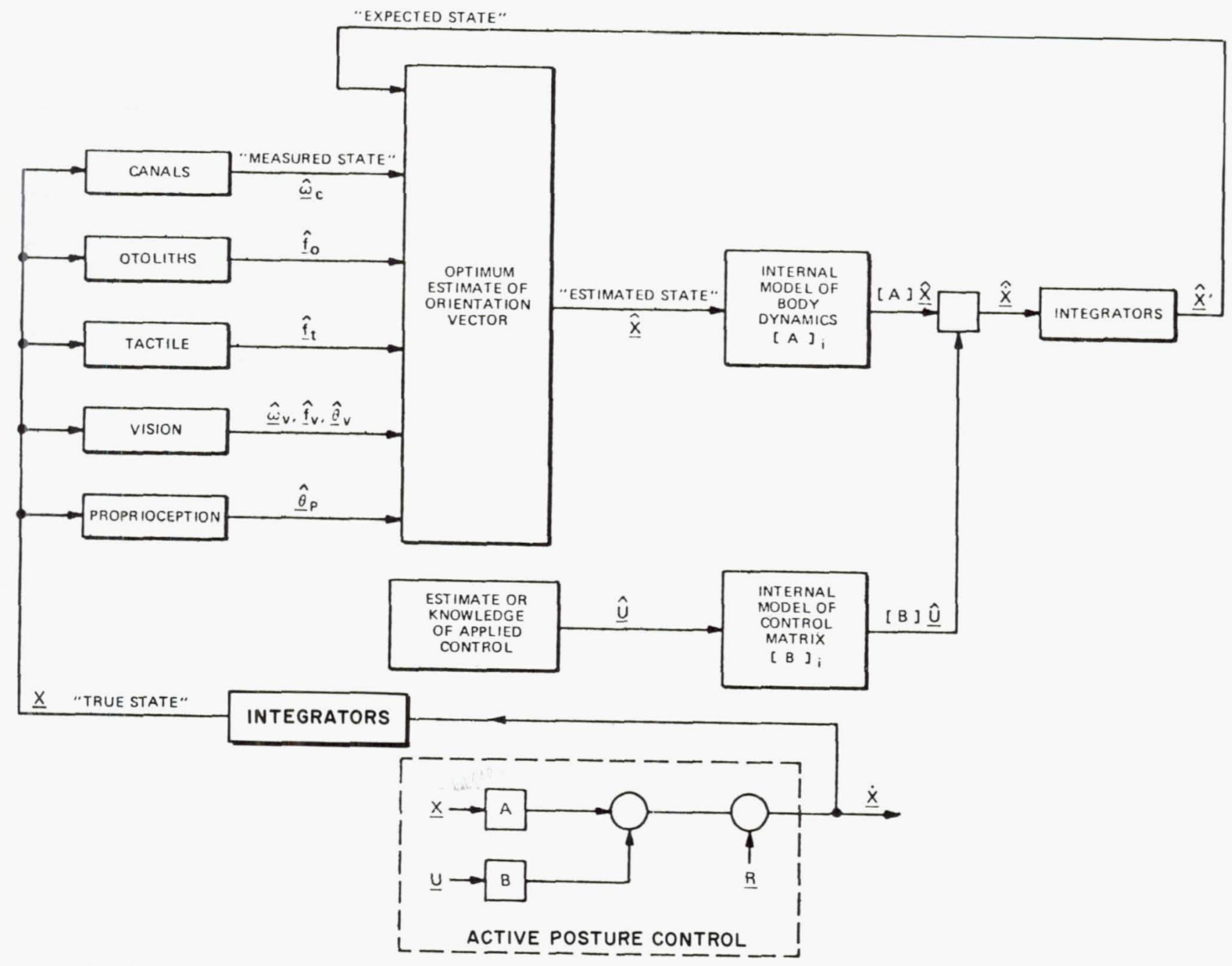

FIG. 2. Schematic representation of spatial orientation process. "True state" vector, $\underline{X}$, consisting of linear and angular positions and velocities, is produced by changes resulting from three sources: unforced behavior of body, $\underline{\hat{x}}$ processed by A, commanded body changes, commands $\underline{U}$ processed by $\mathrm{B}$, and unmeasured disturbances, $\underline{\boldsymbol{R}}$. Various sensors are each responsive, especially to one or more components of measured state. Symbol "N" indicates an estimate of the vector; $\omega$, angular velocity; $f$, specific force, gravity minus linear accleration; $\theta$, angle. Measured state signals are combined with "expected state," $\underline{\underline{\hat{X}}}$, derived from a presumed internal model of the body, in optimum estimator to produce estimate of orientation, $\underline{\hat{X}}$. [From Young (174).]

ducers only for stimulation frequencies so low (less than $0.1 \mathrm{~Hz}$ ) as to lie in the range of man-made transportation and amusement devices rather than in the normal physiological range.

\section{Otolith Organs}

The otolith organs, discussed more fully in the chapter by Goldberg and Fernández in this Handbook, are linear accelerometers and, as such, respond to linear accelerations and to changes in orientation with respect to the gravity vector. The otolithic membrane in each utriculus and sacculus, made more dense than the surrounding endolymph fluid by the presence of numerous calcite crystals, slides downhill when the head is tilted and lags behind when the head is accelerated with respect to inertial space. Between the utricles and saccules these organs represent acceleration sensitivity in all three dimensions. They are the principal nonvisual determinants of static orientation with respect to the vertical. In conjunction with the vertical semicircular canals they also act to signal changes in orientation and to initiate corrective postural responses. The implied ambiguity in the use of otolith information for static orientation (physical devices cannot distinguish between linear acceleration and gravity) is normally solved by interpreting the otolith signals on the basis of other sensory information, including semicircular canal afferents.

\section{Somatosensory Cues}

Although the primary nonvisual orientation cues in humans are vestibular in origin, the influence of non- 
vestibular signals must not be ignored. The classic nonvestibular righting reflexes seen in labyrinthectomized animals, in response to asymmetric tactile cues, are dramatic examples (156). In humans the ability of labyrinthine-defective subjects to retain orientation and balance, even in the dark, is ample indication of the potential utility of nonvestibular orientation cues. For those rare situations in which vestibular cues are altered temporarily, such as in the weightless condition of space flight, tactile and proprioceptive cues appear to substitute for some aspect of otolith signals. The proprioceptive and tactile cues are of essentially three kinds: pressure cues, limb position signals, and muscle length and tension afferents.

The quantitative relationships between sensory characteristics of surface and deep pressure somatic endings and perception has received relatively little attention. The high-frequency pass band of many of the pressure endings corresponds to perception of vibration rather than phase-dependent orientation oscillations. There is, however, a remarkable similarity between the sensitivity curves of various somatosensory endings as a function of vibration intensity and frequency and the corresponding psychophysical curves for detection of local vibration.

Pacinian corpuscles are deep pressure sensors with a rather distinctive morphology. They are encapsulated by cylindrical fluid-filled sheaths, or lamellae, that prevent static deformation from reaching the hard elliptical core. It is this dendritic core that is actually responsible for producing generator current, and thus the capsule acts as a mechanical high-pass filter of deformation stimuli. Because of the mechanical structure of the capsule, when a pressure is released the core tends to distend along the orthogonal axes and also to produce a generator current. Pacinian corpuscles show no significant static response to compression stimuli and are quickly adapting, displaying a time constant of $1-10 \mathrm{~ms}$.

Being deep pressure sensors, Pacinian corpuscles have a fairly wide receptive field and respond to stimuli not directly above the corpuscle. They are exquisitely sensitive and respond to displacements as small as $10 \mu \mathrm{m}$ (105).

There are also two types of pressure sensors located close to the surface of the skin. Type I receptors are formed by myelinated fibers that end in Merkel cells near the surface of the skin. Merkel cells are found within domelike elevations of the epidermis between hair follicles known as Iggo corpuscles. Type I receptors exhibit highly focused receptive fields and respond only to direct stimulation of the touch corpuscle. They respond dynamically to stimuli as small as $1-5 \mathrm{~mm}$ of skin displacement. The step response adapts with time constants of about $1 \mathrm{~s}$ and about $30 \mathrm{~s}$. Type I receptors show a static response, but it is characterized by a highly irregular afferent rate. These fibers usually do not exhibit any resting discharge (see ref. 92).

Type II receptors are formed by myelinated fibers ending in lightly encapsulated Ruffini endings. The end organ is situated in the dermis but is not as close to the skin as the type I receptors. Their linear transfer function to skin displacement can be fit with three adaptation time constants of approximately 1, 5, and $20 \mathrm{~s}$. Type II receptors exhibit a regular static response as well as a regular resting discharge and have a relatively wide receptive field responsive to stretch (see ref. 29).

Psychophysical studies of human threshold to skin vibration suggests two receptor populations, one population being sensitive to very high frequencies. These results are consistent with quickly adapting Pacinian corpuscles beneath the dermis and more slowly adapting cutaneous receptors, as described in neurophysiological studies.

Psychophysical studies have also shown that the threshold tends to decrease with increasing stimulus area $(107,161)$. The principal function of somatosensory information in the normal system is apparently for rapid detection of changes in surface force, which corresponds to changes in acceleration under most circumstances (122).

\section{Limb Position}

Body-orientation perception also depends heavily on the perceived orientation of the various joint angles. Not only are limb-position angles relevant to postural control, but the orientation of the head with respect to the trunk is especially critical. Because vestibular and visual cues are measured in a headfixed coordinate system and yet the postural reactions to the appropriate muscles must be in a different coordinate system, it is obvious that the relative orientation of the head, trunk, and limbs must be known reasonably accurately. The common notion that these joint angles are sensed exclusively or even primarily through the joint receptors in the joint capsules has been called into question. Although gross and monotonic signals relating joint angle to capsule sensor afferents certainly exist, they may not account for the accurate perception of limb position that is clearly at work in many human tasks. Among other considerations, these capsule receptors, which are really pressure receptors, are influenced by total force across the limb, which in turn is subject to external variables.

The muscle receptors, muscle spindle afferents, and Golgi tendon organs, once believed to play no role in proprioception or kinesthesis, are now generally acknowledged to be of importance in determining limb position and human spatial orientation. Muscle spindle afferents of several types signal the length of intrafusal muscle fibers, which in turn relate to overall muscle length and its rate of change, as well as to the intended or regulated muscle length commanded by the $\gamma$-motor-control signals. Golgi tendon organs are capable of monitoring total force in a portion of a muscle and therefore indirectly of monitoring the weight of the head or a limb. Both spindle and tendon organs can therefore play two roles relative to human 
spatial orientation. As force sensors they can serve as additional linear accelerometers in which the force required to maintain head or limb position is a measure of both orientation with respect to gravity and net linear acceleration. One of the cues to a pilot that he is in a high-acceleration turn, for example, is that his head feels heavy and that it is an effort to maintain his arm position. Additionally, in the nonaccelerating environment these signals can augment the joint capsule receptors in signaling the orientation of the head with respect to the trunk. The effect of artificial spindle stimulation on perception of limb orientation is easily demonstrated in humans by the ability of an externally imposed vibration over a muscle to produce the perception of muscle shortening (65). Supporting physiological evidence comes from the discovery of cortical representations of certain muscle spindle afferents (59). Specific application of vibration to certain postural muscles can produce predictable changes in body orientation. The strength of body-orientation illusions in response to this artificial spindle stimulation is variable among subjects (101).

\section{PSYCHOPHYSICAL MEASURES OF PERCEPTION} OF ORIENTATION AND MOTION

Throughout this chapter, reference is made to experimental results about subjective orientation, or the perception of motion. Obviously we have no means of measuring what the subject thinks or feels except by his own indications. (Electrical or magnetic evoked responses are not current useful alternatives for this purpose.) Two general categories of quantitative indications of orientation and motion perception are used. The first of these makes use of magnitude estimation either as a concurrent or retrospective judgment. The notion of attaching quantitative estimates to sensation magnitude and relating them in turn to objective physical stimuli builds on the early work of Weber (166a), modified by Fechner (48a), leading to the logarithmic sensitivity notion that a just noticeable difference (JND) in stimulus is proportional to the stimulus level. In performing magnitude estimations, quantitative subjective estimates of a scalar quantity are made in relation to a previously learned or revealed reference scale. The techniques of appropriate magnitude estimation methodology are reviewed by Stevens $(149,150)$ and by Poulton (134). These techniques include pinning down the end and the midpoint of the scale, refreshing the subject with calibration exposures to the known stimuli, and avoiding placing test stimuli close to the ends of the reference scale. Ratio estimates are particularly useful. When the estimate is made during the stimulus, it is known as a concurrent estimate. However, the subject may also wait until the stimulus is completed to judge how fast he was moving or how far he had moved (integrating the subjective velocity judgment), in which case it may be referred to as a retrospective estimate. Guedry et al. (83) have demonstrated how concurrent and retrospective estimates can lead to very different magnitude estimates, using the situation of triangular velocity wave forms for rotation about a vertical axis as an example. Retrospective subjective angular displacement judgment yields total displacement estimates reasonably close to true angular displacements and is predicted well by the torsion pendulum model referred to in the chapter by Goldberg and Fernández in this Handbook. When the same type of displacement estimate is made continuously, concurrent with the stimulus, and the subject attempts to always keep the pointer stationary in space, the total displacement is substantially less, as indicated in Figure 3. This possible influence of the concurrent magnitude estimation task on the perception being measured is not often considered, but it may be of some significance in explaining certain perception results. A task of magnitude estimation need not involve an arbitrary scale or even practice on the reference scale. For example, many of the results on tilt perception, including the rod and frame test and the tilted-room experiments (170), rely on experiments in which the subject is required to align a visually observed line or a calibrated rod to the perceived vertical, and the angle between this alignment and the true vertical is taken as the error in the perceived tilt. No extensive instruction on the meaning of the vertical as a reference scale is required.

An alternate method of quantifying perception is a response-nulling technique. In the nulling method, the subject actively controls his own stimulus in order to return himself to a subjective reference level. For example, to assess the magnitude of visually induced tilt the subject may be required to actively orient

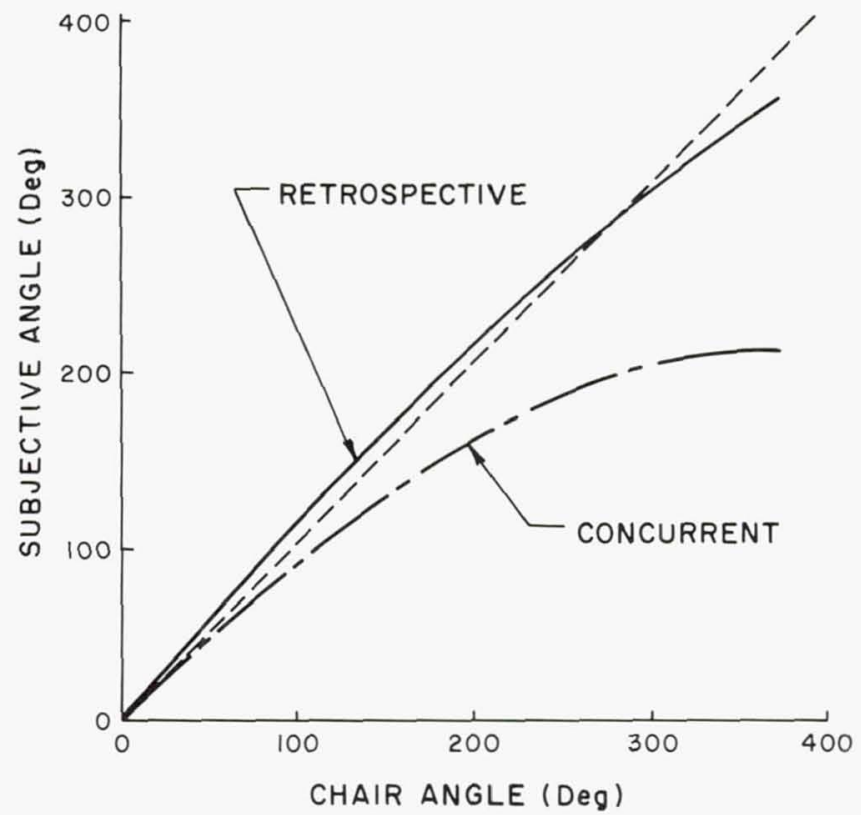

FIG. 3. Subjective estimates of angular displacement (produced by triangular velocity wave form) are greater for retrospective than for concurrent estimates. [Adapted from Guedry (76).] 
himself to a posture that he perceives to be upright. Alternatively, the subject might control the angular velocity of a visual field or his own actual inertial rotation in the presence of some test stimulus until he feels that he is stationary. In a final variation, that of sequential matching, the subject attempts to give a quantitative indication of his perception of motion or tilt, induced by one stimulus, by subsequently matching the sensation through another stimulus.

The nulling method and the sequential matching method share the advantage of avoiding substantial confusion about the subject's interpretation of which physical variable is to be estimated.

\section{ANGULAR ACCELERATION}

\section{Rotation in Dark}

The most frequently studied motion sensation results from rotation about a vertical axis in the dark. The dizziness that comes from opening one's eyes during constant-velocity turning or after having been brought to an abrupt stop is a common childhood experience. It is easily explainable on the basis of stimulation of the semicircular canals in a single plane. When the head is pitched approximately $25^{\circ}$ forward from the errect position the human horizonatal semicircular canals lie approximately in the horizontal plane, and rotation about a vertical axis stimulates primarily but not exclusively these semicircular canals. The corresponding semicircular canals are neither precisely coplanar nor very close to orthogonality with the other canals in each labyrinth (12). When the axis lies through the head, centripetal acceleration effects on the otoliths may be neglected. The simplicity of this stimulus axis has made it particularly attractive for use in indirect measurements of semicircular canal mechanics. The earliest of these attempts at "cupulometry" was accomplished with steps of angular velocity. The torsion-pendulum model, discussed in Torsion-Pendulum Model, p. 1030, would predict a rapid rise and a slow exponential decay to null following a step in angular velocity, but the actual experience is somewhat different. Neither subjective measurements nor nystagmus can reveal the short time constant of fractions of a second. The subjective response does, however, decay right through zero to yield a period of reversed sensation. Subjective response to a long-duration constant velocity step, followed by a sudden return to zero angular velocity, is shown in Figure 4. The initial decline in subjective velocity is nearly exponential. The similarity between this response and the predicted return of the cupula according to the torsion pendulum equation led to the use of subjective sensation decline, as well as of nystagmus decline, following velocity steps as a means of estimating the long time constant, $\Pi / \Delta$ - the ratio between viscous and elastic coefficients. The differing slopes of the response declines for subjective sensation and for nystagmus, however, indicate the involvement of more than cupula dynamics alone (72).

Models for vestibular adaptation to horizontal rotation allow for the possibilities that cupula responses are not mirrored exactly in either nystagmus or subjective sensation. (Recent developments in single-unit recording of first-order afferents in some species and of recordings from the vestibular nucleus and thalamus of other species of monkeys lead to the current view that the time course of cupula response, which may be as short as $5-7 \mathrm{~s}$ in the monkey, is lengthened to give a considerably longer response time at the level of the vestibular nucleus (21)). This longer time constant may be approximately $16-20 \mathrm{~s}$ and reflects rather accurately the time constant of decay of vestibular

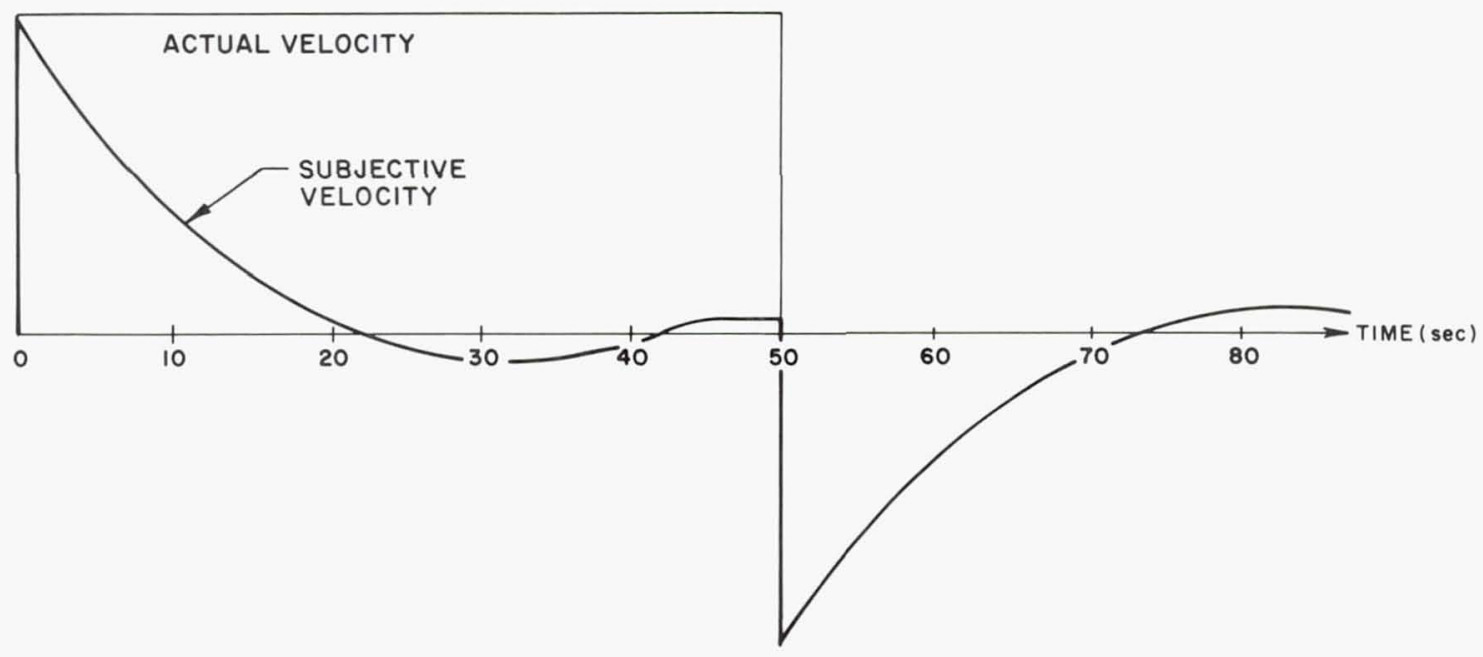

FIG. 4. Subjective angular velocity decays and may reverse during prolonged constant-velocity rotation. Sudden stop after prolonged rotation elicits transient, oppositely directed, postrotatory response. 
nystagmus. Subjective sensation in man is known to decrease with a shorter time constant than that of vestibular nystagmus.

Yet another difference in the dynamic responses observed from afferent records from those seen in nystagmus recordings or in perception responses occurs in rotation about other than the $z$-axis. Examination of the time course of postrotatory sensation or nystagmus for rotations about each of the three principal body axes ( $x, y$, and $z$ ), always about a vertical axis, reveals a significant difference between axes, provided the stimulus is of long enough duration (7, $34,76,82,112,114)$. The long time constant for rotations about the body pitch axis and roll axis for a vertical axis are approximately one-half as long as the time constants for rotation about the body yaw axis. Investigations of the outputs of the individual semicircular canals in squirrel monkeys, however, reveal no such differences in time constants between axes (49). Once again it appears that higher center processing alters the effective time constant from that received from the afferent signal. Certainly the movements normally encountered in pitch and roll, even about a vertical axis, are of briefer duration than for yaw. An integration time constant of more than a few seconds would not be required to reproduce the pitch and roll transient movement faithfully.

\section{Active Versus Passive Movement}

The preceding discussion of postrotatory sensation is applicable strictly to the case where a subject is passively rotated and stopped. Under conditions of active rotation, knowledge of the rotation situation and information supporting the perception of continued rotation at constant velocity may change the perception. In one extreme example, it has been demonstrated that subjects who maintain the sustained angular rotation about a vertical axis by active movements and then are suddenly decelerated generally show postrotatory sensations in the same direction as the original sensation, even though their postrotatory nystagmus reversed direction, just as for passive rotation $(39,80)$. Sensory responses due to active movements are interpreted differently from those due to passive movements. One simple method of thinking of this effect is that active movements provide another and very powerful measurement, consistent with the "efferent copy" concept of von Holst and Mittelstaedt (162) and of others. When the subject "knows what movement was intended" on the basis of his active control, sensory information and particularly conflicting sensory information may be reinterpreted or discarded when inappropriate.

\section{Torsion-Pendulum Model}

The angular-velocity perception resulting from stimulation of a pair of parallel semicircular canals can be related to the mechanics of semicircular canal transduction, to the afferent firing rate of primary vestibular neurons, and to central neuronal activity. Functional interpretation of Steinhausen's (148) observations and subsequent formalization by van Egmond et al. (160) of semicircular canal function in terms of a torsion-pendulum model was based on correlation of the physical model with the time course of subjective perception for rotation of the subject about a vertical axis. Subsequent investigations, especially the direct measurement of first-order afferent signals (49) and central vestibular neurons (115), have revised the earlier view that canal afferents directly drive the slow phase of nystagmus or the subjective angular velocity. Nevertheless, the concept of a relationship between the time course of cupula deflection and the subjective sensation remains a valuable one.

The essence of the torsional pendulum model is spelled out in the chapter by Goldberg and Fernández in this Handbook. To recall, endolymph displacement, $\xi$, is related to head acceleration (with respect to inertial space) in the plane of the canal by the equation

$$
\frac{\Theta \mathrm{d}^{2} \xi}{\mathrm{d} t^{2}}+\frac{\Pi \mathrm{d} \xi}{\mathrm{d} t}+\Delta \xi=\Theta \alpha(t)
$$

where $\theta$ is the moment of inertia of the endolymph ring, including the fluid in the ampulla and utricular sac; $\Pi$ is the viscous drag coefficient; $\Delta$ is the spring constant; and $\alpha$ is the angular accleration of the head. Because of the high ratio of viscous damping of endolymph flow in the semicircular canal $\Pi$ to the moment of inertia of the ring of endolymph $\theta$ the inertial reaction torque on the ring of fluid attributable to its acceleration with respect to inertial space, $\Theta\left[\left(\mathrm{d}^{2} \xi / \mathrm{d} t^{2}\right)\right.$ $-\alpha(t)]$, is very quickly balanced by the viscous damping torque, $\Pi \mathrm{d} \xi / \mathrm{d} t$. The time constant associated with this process is the short time constant, $\tau_{\mathrm{S}}=\theta / \Pi$, referred to in the chapter by Goldberg and Fernández in this Handbook, with a value on the order of 3-5 ms. Within three time constants (9-15 ms) the torque balance is $99 \%$ complete, and the endolymph flow, $\mathrm{d} \xi / \mathrm{d} t$, is proportional to the head acceleration $\alpha(t)$. Consequently endolymph displacement $\xi$ is then proportional to the head angular velocity, $\int_{0}^{t} \alpha(t) \mathrm{d} t$. To the extent that the change in firing rate of canal afferents reflects endolymph displacement, or more properly shear force across the subcupula space of the crista, the afferents signal head velocity for head motion durations much longer than $\tau_{2}$ and shorter than $\tau_{1}$. The long time constant of the semicircular canals, $\tau_{\mathrm{L}}=\Pi / \Delta \simeq 5$ to $15 \mathrm{~s}$, is attributable to the relatively weak elastic-restoring torques of the cupula and membranous canal that oppose any deflection of endolymph by a spring torque $\Delta \xi$. The weak restoring forces must oppose the substantial damping $\Pi \mathrm{d} \xi / \mathrm{d} t$ in returning the cupula and the hair cells to their original position. For acceleration stimulus periods comparable to or longer than $\tau_{\mathrm{L}}$, however, these restoring forces are significant. Consequently for a step of angular acceleration after the first few milliseconds the theo- 
retical endolymph displacement increases according to the equation

$$
\xi_{1}(t)=(\theta / \Delta) \alpha\left[1-\exp \left(-t / \tau_{\mathrm{L}}\right)\right]
$$

as discussed in the chapter by Goldberg and Fernández in this Handbook. If the torsion-pendulum model entirely explained the dynamics of peripheral neurons, central vestibular neurons, and perception of velocity, the perceived angular velocity following the onset of a sustained constant acceleration in the dark would resemble the exponential equation (Eq. 2). In fact, the actual time course of subjective velocity about a vertical axis, as shown in Figure 5, departs from a torsion pendulum only after approximately $30 \mathrm{~s}$. For brief accelerations $(0.6-6 \mathrm{~s})$, the perceived velocity is roughly proportional to the true velocity of the head. For such motions, which cover the physiological range of head motion $(0.1-1.0 \mathrm{~Hz})$, the integrating property of the endolymph viscous damping makes the semicircular canals resemble integrating accelerometers, and they yield accurate velocity estimates in daily activity. [Absolute measures of subjective velocity are difficult to obtain. Authors frequently report the indicated velocity to be up to $50 \%$ greater than the actual velocity during the first $15 \mathrm{~s}$ of exposure $(74,78)$.] Of course these velocity estimates must be integrated once more by the central nervous system to produce estimates of angular orientation-even as used in the "90-degree turning points" method of subjective rotation-magnitude estimation. Errors in this second integration, including scaling, loss of initial conditions, and imperfect integration, lead to methodological problems in judgment of azimuth orientations in the laboratory (see, for example, ref. 81).

For sustained accelerations of medium duration (6-25 s), the perceived velocity gradually drops below the linearly increasing head velocity and approaches an asymptotic constant velocity proportional to the imposed acceleration, with a time constant of $12-15 \mathrm{~s}$. The rise to a constant level during a constant acceleration is basically similar to the step response of the torsion-pendulum model and the nonadapting semicircular canal afferents (see Fig. 9 in the chapter by Goldberg and Fernández in this Handbook). It would be a mistake, however, to assume that cupula pressure or displacement is directly encoded to yield subjective velocity. First of all, the time constant of the rise (12$15 \mathrm{~s}$ ) is probably longer than human horizontal canal response, based only on dimensional analysis [ $(38$, 116); I. S. Curthoys and C. M. Oman, unpublished observations] and on the known squirrel monkey canal long time constant of approximately $5 \mathrm{~s}$. Second, com-

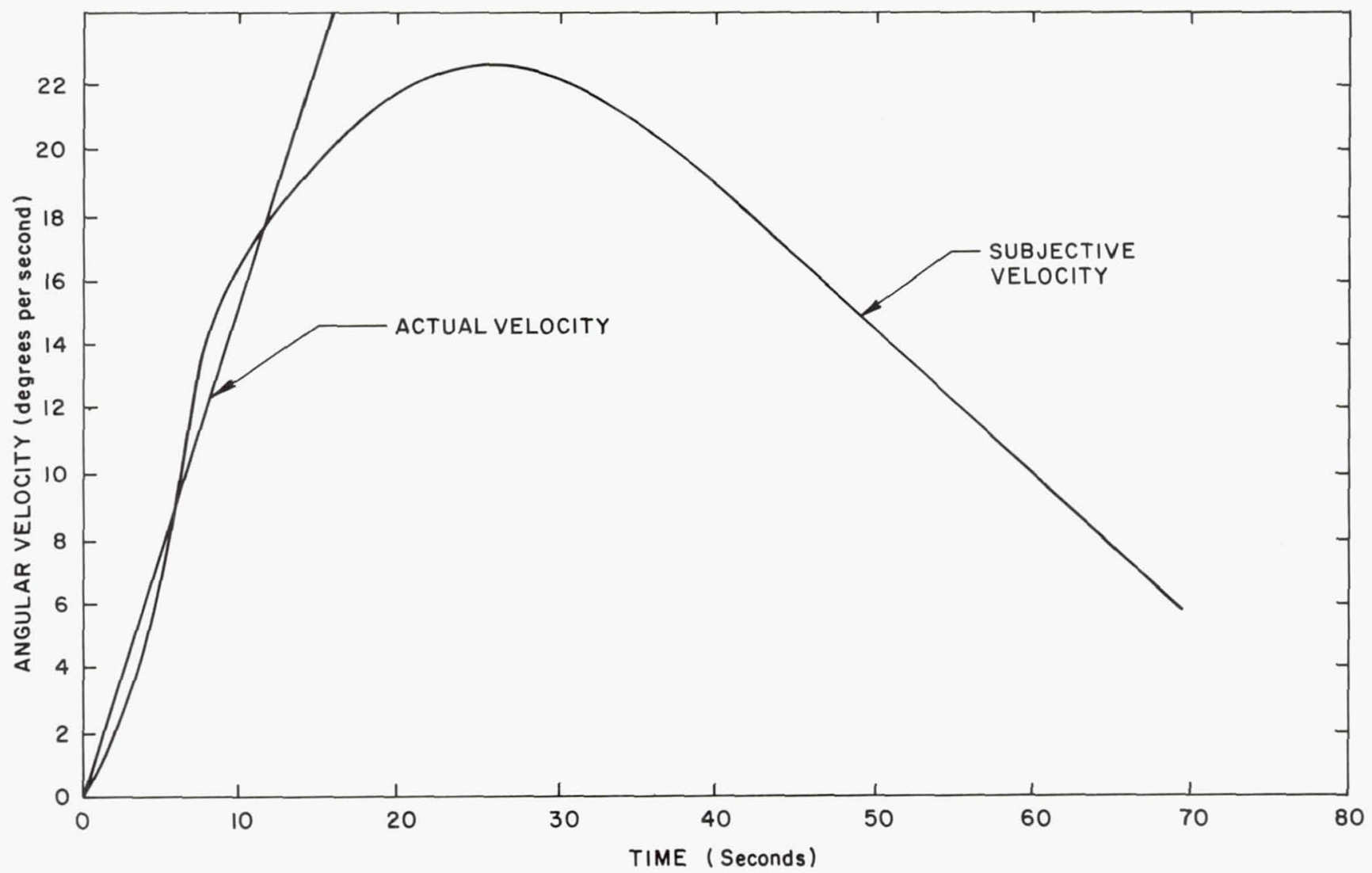

FIG. 5. Subjective velocity follows actual velocity only for $1 \mathrm{st} 10 \mathrm{~s}$ of constant angular acceleration, then plateaus and actually decays. [Adapted from Guedry and Lauver (79a).] 
missural pathways from the contralateral labyrinth (inhibitory with delay) as well as vestibular efferent signals (partially disrupted by anesthesia) probably lengthen the apparent time constant to approximately equal the value of nearly $20 \mathrm{~s}$ seen in the slow phase of vestibular nystagmus. One cannot yet eliminate the possibility that those (few) fibers that go directly from the eighth nerve to the cerebellum without first synapsing in the vestibular nucleus are responsible for rotation perception, thereby invalidating the above argument. Recordings of unit activity in the monkey thalamus $(24,25)$ also show an effective time constant for responses to angular acceleration which, although longer than the canal afferents, is shorter than the time constant associated with nystagmus.

\section{Thresholds}

To put into perspective the various experimental reports of acceleration-sensation threshold, time to detect, and duration of sensation, it is useful to return once more to the predicted cupula deflection following from the torsion-pendulum theory (76).
According to the simplified threshold notion of minimum detectable sensation related to threshold cupula position or pressure, the responses to acceleration and velocity steps are implicit in the simple exponential wave forms of Figure $6 A$ (acceleration step) and Figure $6 B$ (velocity step). For an acceleration step, the time course of calculated cupula deflection is

$$
x(t)=\alpha \tau_{\mathrm{S}} \tau_{\mathrm{L}}\left[1-\exp \left(-t / \tau_{\mathrm{L}}\right)\right]
$$

and the time required to reach a given threshold level $\left(x_{\min }\right)$ is given by

$$
t_{\text {detect }}=\tau_{\mathrm{L}} \ln \left[\tau_{\mathrm{S}} \tau_{\mathrm{L}} \alpha /\left(\tau_{\mathrm{S}} \tau_{\mathrm{L}} \alpha-x_{\min }\right)\right]
$$

Clearly, for $\alpha \leq x_{\min } / \tau_{\mathrm{S}} \tau_{\mathrm{L}}$ the time to detect becomes infinite, thus defining a threshold acceleration $\alpha_{\min }$. In fact, the curve shown in Figure 7 matches the formulation extremely well. Note further that for all but the lowest (near threshold) accelerations, the curve is close to the hyperbola, $\alpha t_{\text {detect }}=C$ (a constant). Another way of stating this approximation is: for stimulus durations that are short relative to $\tau_{\mathrm{L}}$, the cupula deflection or pressure rises almost linearly with time following an acceleration step, and so the threshold
FIG. 6. Normalized torsion-pendulum response, $\mathrm{X}$, for system with long time constant, $\tau_{\mathrm{L}}=15 \mathrm{~s} . A$ : rising response to acceleration step is detected when it reaches $\mathrm{X}_{\min }$. $B$ : decaying response following velocity step has duration $t_{\mu}$.
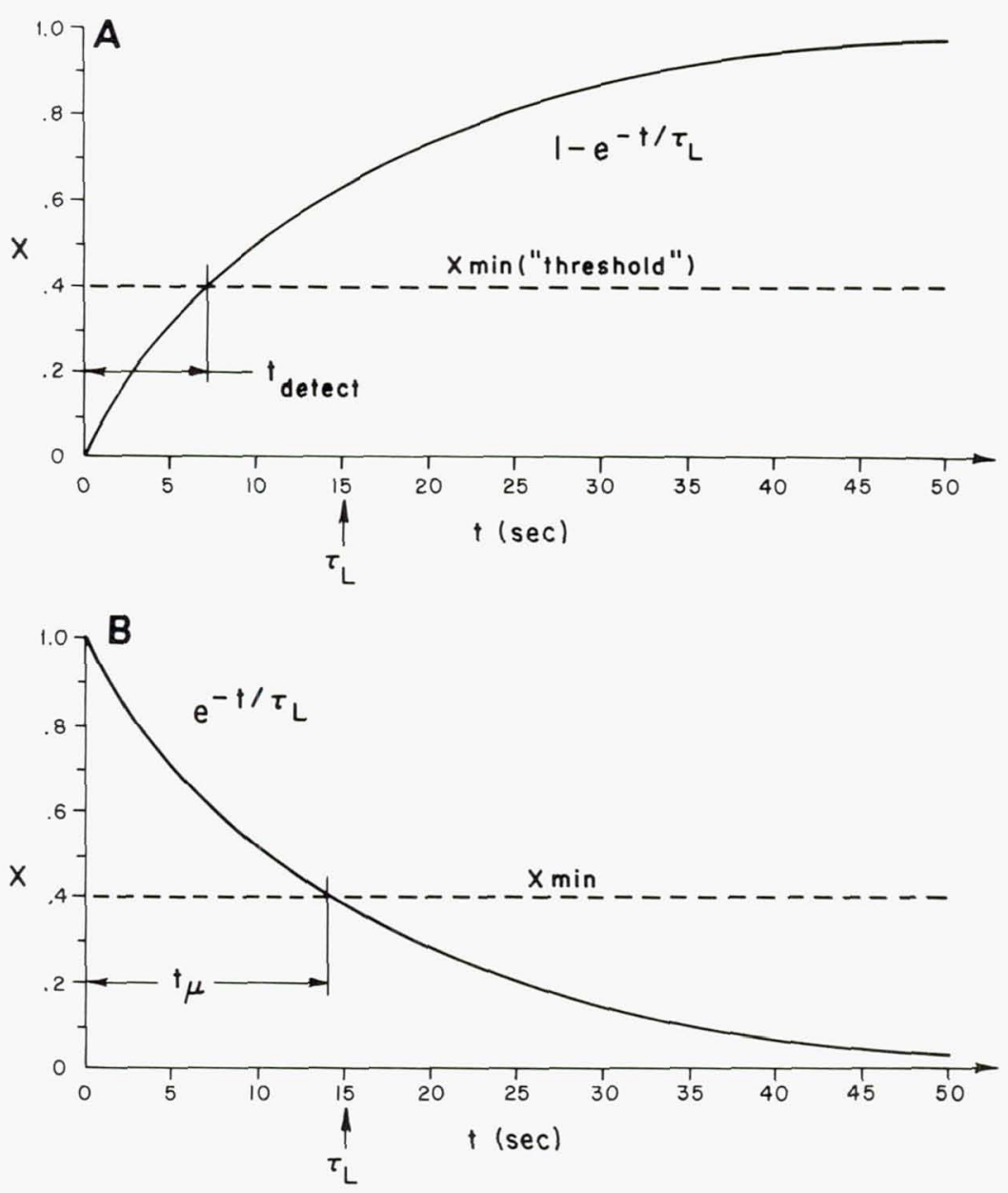


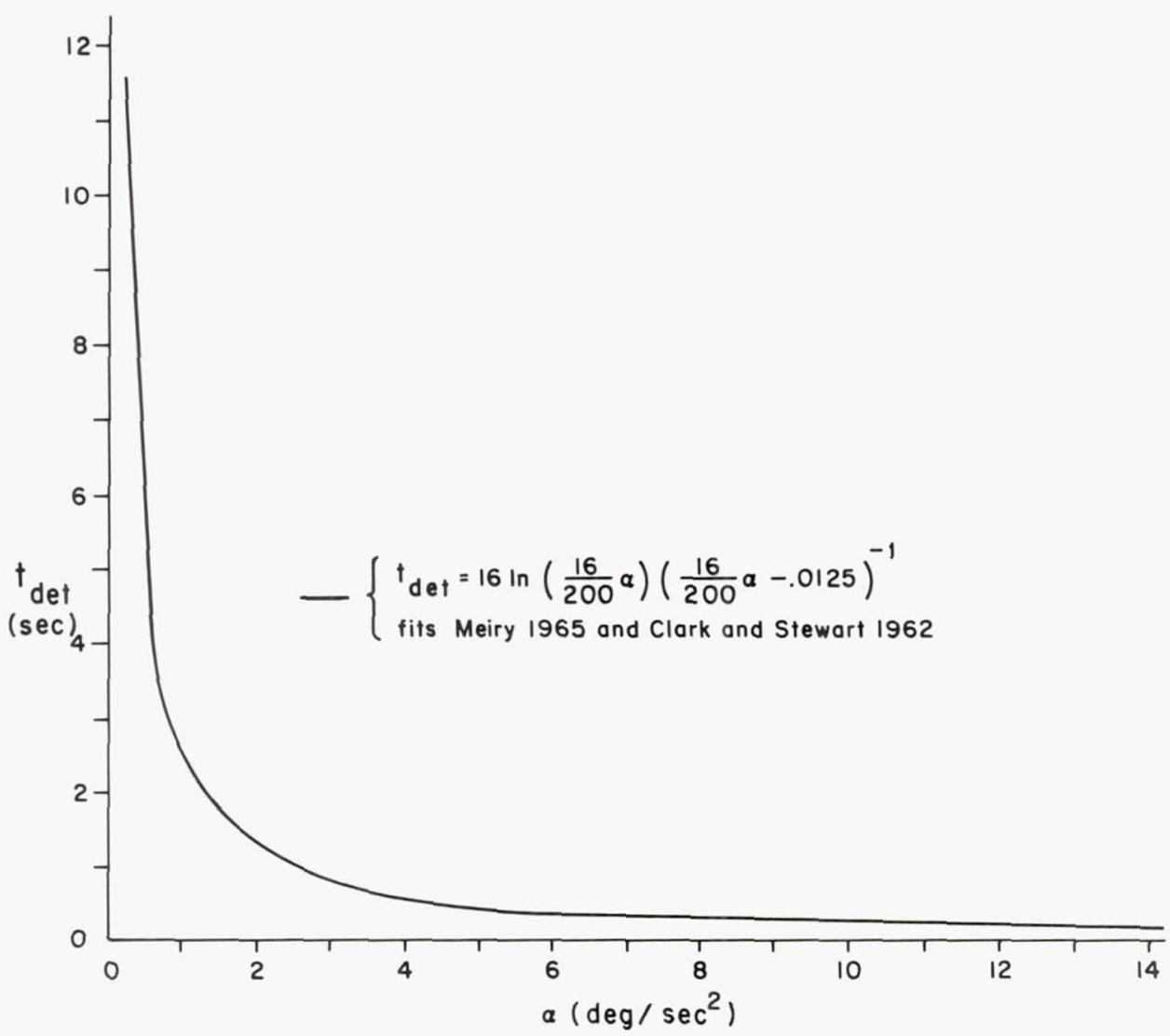

FIG. 7. Time to detect, $t_{\text {det }}$, step of yaw angular acceleration of magnitude $\alpha$ increases sharply for acceleration levels less than $2-3 \mathrm{deg} / \mathrm{s}^{2}$. [Adapted from Guedry (76).]

level is reached when a certain critical velocity is attained, independent of the acceleration amplitude. This critical velocity, known as the Mulder product, is merely the minimum detectable angular velocity for brief steps of acceleration

$$
\omega_{\min }=\alpha_{\min } \tau_{\mathrm{L}}
$$

or $\omega_{\min } \simeq 2.5 \mathrm{deg} / \mathrm{s}$, assuming $\tau_{\mathrm{L}}=10 \mathrm{~s}, \alpha_{\min }=0.25$ $\mathrm{deg} / \mathrm{s}^{2}$, or $\tau_{\mathrm{L}}=12.5 \mathrm{~s}, \alpha_{\min }=0.20 \mathrm{deg} / \mathrm{s}^{2}$. For lower accelerations, of the order of only two or three times threshold (less than $1 \mathrm{deg} / \mathrm{s}^{2}$ ), even the theoretical cupula deflection departs significantly from linear growth long before a threshold is crossed. Consequently, for small accelerations $t_{\text {detect }}$ is longer than predicted by the Mulder product and is given by Equation 4.

Another use of the concept of a cupula-deflection threshold is in interpreting postrotatory motion sensation or nystagmus. As indicated in Figure $6 B$, the calculated cupula deflection following a sudden change in angular velocity, such as a stop after sustained constant velocity rotation of $1 \mathrm{~min}$, is a rapid peak followed by an exponential decay to zero with time constant $\tau_{\mathrm{L}}$

$$
x(t)=\omega \tau_{\mathrm{S}} \exp \left(-t / \tau_{\mathrm{L}}\right)
$$

where $\omega$ is the size of the angular velocity step. On the assumption of a cupula-deflection threshold $x_{\min }$, the sensation of rotation has a duration $t_{\mu}$ (76) found from the equation

$$
x_{\min }=\omega \tau_{\mathrm{S}} \exp \left(-t_{\mu} / \tau_{\mathrm{L}}\right)
$$

to yield

$$
t_{\mu}=\tau_{\mathrm{L}}\left[\ln \omega+\ln \left(\tau_{\mathrm{S}} / x_{\min }\right)\right]
$$

The first term of Equation 6 indicates that the duration of sensation would be proportional to the log of the velocity step and that the constant of proportionality (slope) would be the long-time constant $\tau_{\mathrm{L}}$. Such plots of sensation duration versus stimulus velocity for sudden stops, called sensation cupulograms, are roughly straight lines when plotted on semilog paper and were used in early estimates of $\tau_{\mathrm{L}}$, yielding values in the region of $10-15 \mathrm{~s}(28,160)$.

The concept of a threshold value of a physical stimulus for its detection seems simple enough: merely the minimum level of that stimulus or the minimum change that can be reliably and immediately detected. In practice, the notion of threshold for detection of angular acceleration is not nearly as simple. Several problems are associated with the estimate of $\tau_{L}$ from a sensation cupulogram or even from continuous magnitude estimates of velocity. First of all the decay is not really a simple exponential but exhibits adaptation 
and overshoot, as discussed in Adaptation, p. 1035. Therefore, any estimation of a single time constant will be too small if the adaptation effect is neglected. Second, the slope is less steep for the nystagmus cupulogram than for sensation, which is not consistent with a simple interpretation of cupula deflection totally explaining the dynamic response of both sensation and nystagmus. Third, the phenomenon of habituation or reduced response to repeated stimulation can easily lead to a sensation cupulogram with a slope that is too small ( $\tau_{\mathrm{L}}$ too low) if tested with successively increasing velocities or too large if tested with decreasing velocities (28). Threshold or duration measures for nystagmus are typically based on the presence of a fast phase, or beat, rather than the associated slow compensatory eye deviations. The first ocular reactions to low semicircular canal signals are these slow eye deviations, however, and nystagmus represents an overload condition of sorts. Beyond these methodological problems lie some fundamental limitations in considering a hard threshold $x_{\min }$ associated with a minimum cupula deflection. No such threshold has been reported in either individual first-order afferent recordings or unit recordings from neurons in the vestibular nuclei (see the chapter by Goldberg and Fernández in this Handbook.)

Sensation measures always depend on the subjective willingness to risk an opinion based on minimal information. If the response is to be the subject's reporting of a detectable motion, care must be taken to control such variables as the subject's willingness to guess or a tendency for the subject to wait until he is certain before hazarding an opinion. Normally, distinguishing the inherent detectability of a signal from the subject's strategy is accomplished by signal detection theory and by use of receiver operating characteristic curves. In one common implementation, the subject was forced to choose one direction or another for a test at a fixed time after the test was initiated. By convention, the acceleration magnitude that leads to $75 \%$ correct detection is associated with the threshold. For the double-staircase method of threshold determination, the subject is presented with two staircases of stimulus levels, one initially much higher and one much lower than the presumed threshold. After each correct identification, the following stimulus from that staircase is lowered by a fixed ratio and after each error the subsequent stimulus presentation is raised. In this manner the upper staircase descends and the lower staircase ascends toward the threshold level. The two staircases finally cross and recross, yielding an estimate of the actual threshold.

The entire concept of a vestibular rotation threshold is better couched in terms of information or signal detection than in terms of a physical switch. Consider, for example, a combined stimulus pattern, c, consisting of a simultaneous threshold velocity $v_{t}$ and threshold acceleration step $a_{t}$ in the same direction (see Fig. 8). The calculated cupula deflection never exceeds the hypothetical hard threshold level $x_{\min }$ for any of the three stimuli. If the simple concept of a cupula threshold were adequate, the combined stimulus would be

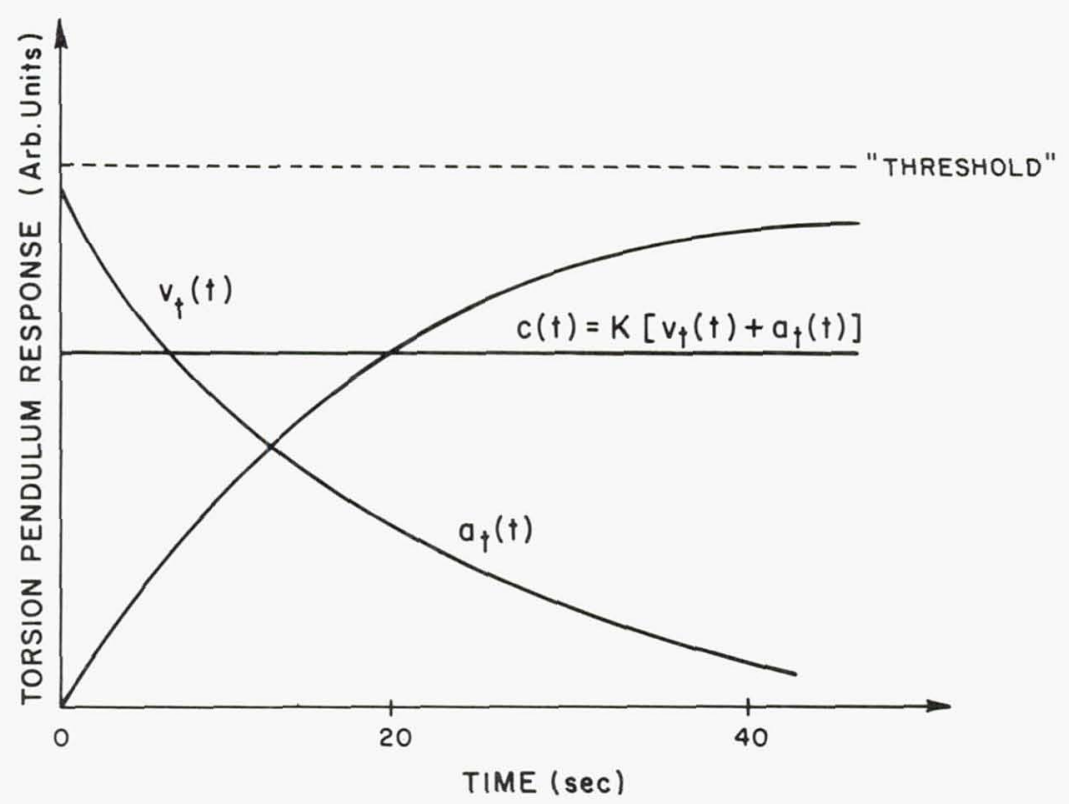

FIG. 8. Theoretical response of the torsion-pendulum cupula model to threshold velocity step, $v_{t}(t)=e^{-t / 18}$, to threshold acceleration step, $a_{t}(t)=1-e^{-t / 18}$, and to a combined stimulus $\mathrm{c}(t)=$ $\mathrm{K}\left[v_{t}(t)+a_{\mathrm{t}}(t)\right]$. In theory, calculated cupula response for $\mathrm{c}(t)$ never exceeds threshold response to velocity or acceleration threshold steps alone and would be undetectable for $K$ as large as unity. Measured thresholds for $\mathrm{c}(t)$ tended to values of $K$ below 0.75 , lending support to signal-detection model rather than hard limit model for threshold. [Adapted from Ormsby (131).] 
just barely detectable or still at threshold when it was the sum of $v_{t}$ and $a_{t}$. Experiments concerning the detectability of various combinations of acceleration impulses $v_{1}$ and steps $a_{1}$ showed that detection thresholds were always lower than predicted on the basis of cupula deflection alone (131). Not only the peak cupula deflection but the duration of its deflection contributed to stimulus detectability, and they support the concept of threshold in terms of a signal-detection process. Recall that the resting discharge rates of both regular and irregular first-order afferents vary from moment to moment in the absence of any stimuli. The signal-detection model assumes that an estimator must identify a probable signal from a noisy process consisting of the parallel firings of many afferents. For signals that are small compared to the noise standard deviation, a longer processing time is required (131). This signal-in-noise theory is consistent with the psychophysical threshold and detection-time measures, and with the absence of any clear threshold in afferent rates. Furthermore it is easily extended to explain experiments in which the detection time is biased by the expected direction or time of an acceleration or by visually induced motion (176).

\section{Oculogyral Illusion}

Thresholds for detecting motion in the presence of a light are one-half to one-tenth the size of those measured in the dark $(31,68,74,91,136,159)$. When the subject is rotated in the dark and has a single light source fixed in front of him, that light source also appears to move relative to the observer. This illusion of movement is referred to as the oculogyral illusion. It was intially explained on the basis of noncompensated slow-phase eye movements associated with vestibular nystagmus. These slow movements in the direction opposite to the acceleration stimulus would produce retinal slip of the observer-fixed target in the same direction as true-target motion relative to the observer in the direction of his acceleration. It was presumed that suppression during fast phases was present. However, a visual target normally inhibits or suppresses vestibular nystagmus, at least below the resolution of most eye-movement measurements, and so the simple eye-movement explanation does not hold. The oculogyral illusion is seen even with afterimages, which permit no retinal slip (173), although Byford (26) reported its absence under stabilized image conditions. The oculogyral illusion may still be caused by attempted eye movements if the process of voluntary visual suppression of vestibular nystagmus involves a reinterpretation of the position of a target fixed in the visual field (168). Although an adequate explanation for the oculogyral illusion is still not at hand, it is generally assumed that it results from a vestibular signal. The labyrinthine information indicates that not only is the observer moving but also that everything fixed relative to him is also in motion in the same direction, including touched objects and sound sources. The part that remains unclear is why the light should appear to move even farther relative to the observer. Although it is impossible to eliminate the argument that the task of observing the light raises alertness levels and consequently lowers the measured level, it appears that the perceived movement of the light itself contributes to this lower threshold (91).

\section{Adaptation}

Although the torsion-pendulum model for mechanical events in the semicircular canal is useful for explaining the short-term mechanical events and for describing in a general sense the dynamic response of perception and nystagmus to brief acceleration stimuli, this model is clearly inadequate for responses that last more than 20-30 s. For example, the torsionpendulum model predicts that the steady response to a sustained constant acceleration is going to be a constant cupula deflection. The observed subjective velocity, however, reaches a peak at $25 \mathrm{~s}$ after the onset of the constant acceleration and then proceeds to plateau and decrease slowly back to zero, as indicated in Figure 5. Slow-phase nystagmus velocity shows a similar behavior, but significantly it does not peak until 80-100 s after the initiation of the acceleration (108). Unless a more complicated mechanical mechanism is accepted for cupula creep back toward its initial conditions, such as underdamped cupula dynamics (27) or sliding of the cupula over the crista (111), or for efferent signals stiffening of the cupula, some manner of neural adaptation must be assumed to explain this behavior. The adaptation seen in longduration responses affects neither the calculations of perceived angular velocity during brief acceleration nor the time to detect any but the lowest accelerations.

Another major difference between the torsion-pendulum predictions and behavioral measures seen is illustrated in the long-duration response to an acceleration impulse or a step change of angular velocity, as shown in Figure 4. The simple torsion-pendulum model predicts a single exponential decay from a peak value back toward zero, with a time constant of $\tau_{\mathrm{L}}$. Both subjective angular velocity and slow-phase nystagmus velocity not only decay back toward zero but also overshoot, resulting in a secondary phase of postrotatory sensation or secondary nystagmus. Once again the time at which the secondary phase occurs is much earlier for sensation (approximately $30 \mathrm{~s}$ ) than for nystagmus, in which case it may not appear for more than a minute. This evidence of adaptation is consistent with the observations from cupulograms, discussed above in this subsection, that the slope of the cupulogram is different for subjective velocity than for nystagmus, leading to the false conclusion of a shorter semicircular canal time constant $\tau_{L}$ based on sensation. An overall black box model, which appears adequate to describe the dynamic relationship among 
angular acceleration, subjective velocity, and nystagmus slow-phase velocity, is given by the transfer function (178)

$$
\frac{\hat{\omega}(s)}{\omega_{\mathrm{i}}(s)}=\frac{K s^{2} e^{-\tau_{\mathrm{d}} s}}{\left(\tau_{\mathrm{S}} s+1\right)\left(\tau_{\mathrm{L}} s+1\right)\left(\tau_{\mathrm{a}} s+1\right)}
$$

where $\hat{\omega}$ is subjective estimate of angular velocity; $\omega_{\mathrm{i}}$ is actual inertial velocity of head; $K$ is proportionality constant; $\tau_{\mathrm{d}}$ is pure delay time $(0.3 \mathrm{~s}$ for subjective sensation); $\tau_{\mathrm{S}}$ is short time constant (on the order of $0.005 \mathrm{~s}) ; \tau_{\mathrm{L}}$ is long time constant $(16 \mathrm{~s}) ; \tau_{\mathrm{a}}$ is adaptation time constant ( $30 \mathrm{~s}$ for subjective sensation, $120 \mathrm{~s}$ for nystagmus); $s$ is the generalized complex variable in Laplace transform notation. Slightly different formulations leading to similar models were independently developed by Malcolm and Melvill-Jones (108) and were discussed in a conceptual manner earlier by others $(73,79)$.

The site of adaptation is not entirely clear. It probably does not take place mechanically in the semicircular canal itself, because only some of the first-order afferents show such adaptation. They are presumably all subject to the same cupula-response dynamics, unless one assumes a complex multimode cupula-de- formation response. (As discussed in the chapter by Goldberg and Fernández in this Handbook, it is primarily among the irregularly discharging neurons that this adaptation is seen.) On the other hand, units located in the vestibular nuclei nearly all show a longer dominant response time $\tau_{\mathrm{L}}$ and an overshoot in the postrotatory phase, similar to that seen in nystagmus response (163). Similar kinds of behavior but with shorter time constants are evident in units of the thalamus and may be related to evidence of adaptation in the pathways for subjective sensation of rotation (24).

Adaptation effects on the frequency response of perception are, as might be expected, evident only at very low frequencies (below $0.01 \mathrm{~Hz}$ ) and consequently are not evident for normal physiological movements. The frequency response for subjective sensation is shown in Figure 9. The gain and phase relationships between the sinusoidal angular velocity of the head (input) and other perceived angular velocity or velocity of the slow phase or vestibular nystagmus (outputs) illustrates the role of the vestibular system in angularvelocity measurement. Over the midfrequency range $(0.1-1.0 \mathrm{~Hz})$ corresponding to most normal head move-

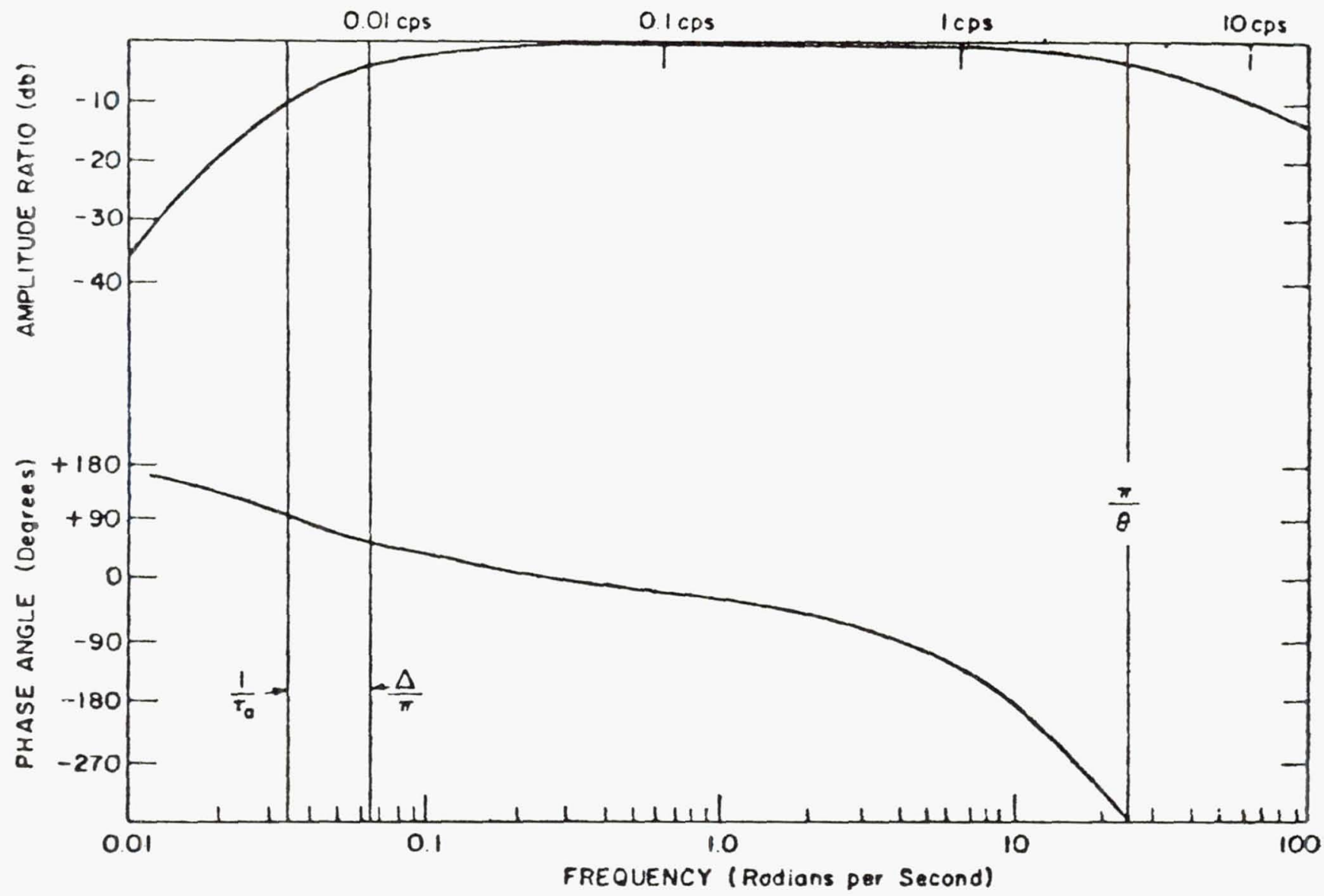

FIG. 9. Frequency response of adaptation model for subjective sensation during yaw angular motion: subjective angular velocity/input angular velocity equals $24.8 e^{-0.3 s} s^{2} /(s+25)(s+0.0625)(s$ + .033). [From Young and Oman (178).] 
ments, the gain is constant and the subjective and nystagmus velocities are roughly in phase with the stimulus. (The eye velocity, of course, is compensatory and opposite in direction to the head movement.) At higher frequencies above $1 \mathrm{~Hz}$, there is evidence that vestibuloocular gain increases to fill in for the failure of pursuit tracking to maintain fixation at higher frequencies. Subjective sensation is virtually meaningless regarding phase at such high frequencies.

For frequencies below $0.1 \mathrm{~Hz}$ the gain declines, and the phase of the subjective and nystagmus velocity response exhibits a substantial lead relative to head velocity. Toward $0.01 \mathrm{~Hz}$ the response velocities are closer to being in phase with the input acceleration than with velocity, which is another way of representing the low-frequency inadequacy of the vestibular system in measuring angular velocity. The adaptation term leads to a phase lead of greater than $90^{\circ}$ at extremely low frequencies (22).

\section{Caloric and Alcohol Effects}

The cupulae of the semicircular canals are normally maintained at a density very close to that of the surrounding endolymph, so that they serve as sensors for angular acceleration but are insensitive to linear acceleration. If absolute neutral buoyancy were maintained (cupula and endolymph of equal density), each semicircular canal afferent activity would be independent of orientation of that canal with respect to the gravity vector. In fact, individual canal units often show a gravity sensitivity in addition to their primary response to angular acceleration $(103,105)$. Presumably any small gravity-sensitive effects of the semicircular canals are either accounted for by central compensation based on otolith inputs or are of such minor influence that they are inconsequential. There are, however, two relatively common situations in which the delicate balance of density between cupula and endolymph is disrupted. One is the clinical test known as caloric stimulation and the other results from ingestion of alcohol.

Caloric stimulation, as introduced by Bárány (2) shortly after the turn of the century, remains among the principal tools of the otolaryngologist for diagnosis of peripheral labyrinthine disorders. The principal mechanism is essentially as described by Bárány. Irrigation of the outer ear by water or air warmer or colder than body temperature introduces a thermal gradient that in time reaches the endolymph of the lateral semicircular canal on the irrigated side. Warm fluid decreases the endolymphatic density (146), causing the now denser cupula to sag in the direction of gravity, as indicated schematically in Figure 10. If the head is placed so that the lateral semicircular canals lie other than in the horizontal plane, a component of gravity acts on the cupula to cause a pressure difference across it or a minor cupula displacement entirely analogous to the sort of cupula displacement resulting from the physiological stimulus of angular acceleration. Detailed measurements and calculations (128, 145) have shown that the torque thresholds associated with generation of nystagmus are equivalent for caloric stimulation and for angular acceleration. This simple theory predicts that the magnitude of the nystagmic response (measured by slow-phase velocity of eye movements) should be proportional to the cosine of the angle between the semicircular canal and the gravity vector. Adaptation occurs to caloric stimuli in a manner somewhat like that to prolonged acceleration stimuli $(13,124)$. Furthermore some basis is pres-

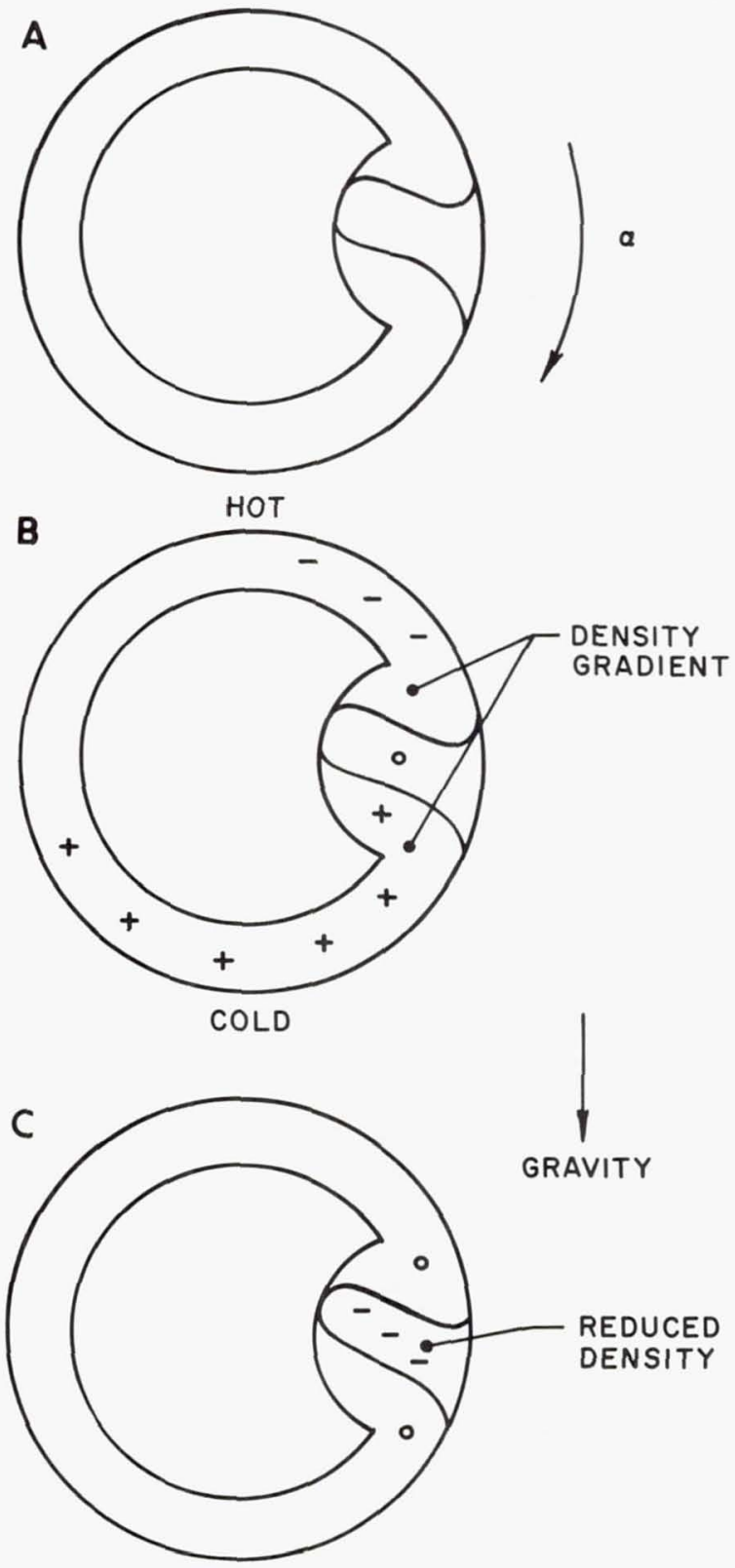

FIG. 10. Exaggerated cupula displacement during $A$ : angular acceleration; $B$ : caloric stimulation; $C$ : first phase of alcohol nystagmus (PAN I). 
ent for assuming that there is a direct thermal effect on nystagmus generation in addition to the influence of gravity. This assumption is supported by Coats and Smith (32), who indicate that the neutral points at which no nystagmus occurs are not found at head positions located at equal angles to those for peak responses (4). The case for direct thermal responses is weakened, however, by the measurements of Kellogg and Graybiel (97) and those of Oosterveld and Van der Laarse (130), who found that caloric responses disappeared during the zero-gravity phase of parabolic flight.

It must be recognized that the subjective sensation of spinning, frequently associated with caloric stimulation in the dark, is a bizarre and nonphysiological one. To maximize the caloric response, the head must be placed so that the lateral semicircular canals lie in a vertical plane. Consequently stimulation of these canals results in a semicircular canal signal, at least from one labyrinth, which indicates rotation about a horizontal axis. For unilateral stimulation this signal is not confirmed by the other labyrinth. Even for bilateral stimulation (hot water in one ear and cold in the other) the semicircular canal signals of rotation about a horizontal axis are in direct conflict with the signals from the utricular otolith organs, which indicate no change in head position with respect to the vertical. It is hardly surprising therefore that the magnitude and time constants of caloric nystagmus depend strongly on static head orientation beyond that necessary to account for the direct physical effect. Head orientation influences the effectiveness of otolith signals in suppressing both visual and semicircular canal inputs.

Alcohol is a commonly used drug that has at least two important effects on vestibular function, as reflected in nystagmic eye movements and perception of orientation. The anecdotal stories of a drunk being unable to walk a straight line (or, more correctly, a given curved line) are well known and reflect the underlying influence of alcohol on both the semicircular canals and on central compensation. The principal effect that has been studied is positional alcohol nystagmus (PAN). It has been known since the early work of Flourens (56) that, when the head is placed with one ear down, nystagmus beating in the direction of that ear takes place during periods of intoxication. The explanation for this phenomenon has only recently been made clear by Money and Myles (121), who demonstrated conclusively that the nystagmus generation (and presumably also the sensation of spinning) was associated with the direct physiological effect of alcohol in initially reducing the density of the cupula with respect to the surrounding endolymph. Once a density difference has been established, the influence of head position and gravity is the same as for caloric stimulation (see Figure 10C). A later effect, known as positional alcohol nystagmus II (PAN II), takes place 4-6 $\mathrm{h}$ after alcohol ingestion, when the direction of the density difference between cupula and endolymph has been reversed. A further effect of alcohol on the central vestibular mechanism is perhaps to eliminate or to reduce the efficacy of central compensation for any minor imperfections or disorders in the peripheral labyrinthine system (8).

\section{LINEAR MOTION AND GRAVITY}

\section{Nature of Linear Accelerometers}

All linear accelerometers rely on the development of inertial reaction forces on a mass and measurement of either these forces or the displacements they create. Because it is essential to the appreciation of the perception of orientation during acceleration that the nature of an accelerometer and of inertial forces is thoroughly understood, an elementary review is included here.

If a 5-gram mass is held stationary in the hand, it requires an upward force on the mass of $5 \times 980$ dyn. This same force presses downward on the hand and can be used to weigh the object. If the mass is being accelerated upward by $980 \mathrm{~cm} / \mathrm{s}^{2}(1 \mathrm{~g})$, then the force downward on the hand is $2 \times 5 \times 980$ dyn, or 10 gramforce (gf). If the mass is allowed to accelerate downward at $980 \mathrm{~cm} / \mathrm{s}^{2}$, then no force is exerted on the supporting hand. If the mass is accelerated to the left at $1 \mathrm{~g}$ while still being supported to prevent it from falling, the force on the hand is the vector sum of 5 gf downward and 5 gf to the right, or $5 \times \sqrt{2}$ gf directed $45^{\circ}$ to the right of down. The force on the support is, in each case, the product of the mass and the vector sum of gravity minus acceleration. This is referred to as the inertial reaction force. When this inertial force is divided by the mass, leaving only the vector ( $\mathrm{g}-$ $\boldsymbol{a}$ ), it is referred to as specific force. Unless the test mass, sometimes called the seismic mass, is surrounded by a fluid of equal density to it so that it remains neutrally buoyant, the action of a specific force would be to press it against its supporting members. These supporting members may be an instrument, springs, muscles, hair cells, or supporting cells.

The otolith organs are by no means the only linear accelerometers in the body. The head, with its point of suspension at the neck several centimeters below the center of mass, serves as an accelerometer. During forward accelerations it snaps back, sometimes with disastrous results. Our whole body serves as a seismic mass supported by the forces through our feet or contact forces from a seat. Thus when a pilot is forced down into his seat by centripetal acceleration during a tight turn, he can use the associated tactile forces to estimate his acceleration as he "flies by the seat of his pants." Our lungs, being considerably less dense than the surrounding tissue, also serve as a potential linear accelerometer. Because the buoyant forces are greater than the inertial reaction forces for the lungs, however, the displacement of the seismic mass is in the direction 
CHAPTER 22: PERCEPTION OF THE BODY IN SPACE

of acceleration. If the cupulae of the semicircular canals are not precisely balanced so as to be of the same density as the surrounding endolymph, as appears to be true when they are infused with alcohol or heavy water, they can serve as the mass for a linear accelerometer and respond with a signal dependent on the orientation of the head with respect to gravity. As late as the nineteenth century it was assumed that human spatial orientation with respect to gravity was determined by shifts of brain tissue or blood in the head on tilting.

Among all the possible linear accelerometers in the human body that might be useful for orientation, the otolith organs play a unique role. As discussed in the chapter by Goldberg and Fernández in this Handbook, the utricular macula, with its collection of hair cells oriented in various polarizations, is primarily sensitive to components of specific force that are parallel to the local plane of the utricular membrane and produce shearing forces on the hair cells. Thus when the human head is pitched $25^{\circ}$ forward from the fully erect position (carried in a normal orientation for walking) with the major planes of the utricle horizontal, the hair cells are in an ideal position to detect any linear acceleration in the horizontal plane. Because the otolithic membrane is displaced by the influence of specific force and not acceleration alone, it is also highly sensitive to the generation of any component of gravity lying along the polarization vectors of the hair cells. In particular, it is ideally suited to detect any tilt of the head forward or backward (pitch) or left or right (roll). Because the hair cells have no way of indicating whether they were displaced by the action of gravity or linear acceleration on the overlying membrane (and, indeed, the equivalence principle of Einstein states that no physical instrument can distinguish between these two equivalent accelerations), it seems clear that this one, approximately planar, accelerometer is faced with providing an ambiguous signal-one that cannot distinguish between a body being accelerated forward or one being pitched backward. Furthermore, the specification of the direction of specific force in a plane does not give a unique orientation of that plane relative to the specific force vector. As a trivial example, signals from the utricular otolith cannot be used to distinguish between right side up and upside down with the head in its normal position relative to the trunk.

The orientation information available from the sacculus is not to be overlooked, however. It is ideally suited for detecting vertical movements when the head is erect, or small rolling movements when the head is tilted at $90^{\circ}$ to the vertical, or small pitch movements when the head is in the prone or supine position.

It might be argued that knowledge of the magnitude as well as direction of the specific force vector relative to the head would be sufficient to determine orientation, but even this is not valid. First of all, there are an infinite number of acceleration and gravitation vector combinations that have a resultant vector magnitude of $1 \mathrm{~g}$. One simple combination is a forward acceleration at $9.8 \mathrm{~m} / \mathrm{s}^{2}$ combined with a free fall vertically at $9.8 \mathrm{~m} / \mathrm{s}^{2}$. More practically, consider the specific force associated with a static head tilt of $1.5^{\circ}$ to the right, which corresponds roughly to the threshold of detectable head tilt. The lateral component of acceleration is $g \sin 1.5=0.026 \mathrm{~g}$. The compressive component in the major plane of the utricles, lying in the plane of the sacculus, is $g \cos 1.5=0.9997 \mathrm{~g}$. This is indistinguishably different from $1 \mathrm{~g}$ and is not detectable by the sacculus units. Thus, based on information from the otolith organs alone, there would be no way of distinguishing between the tilt of $1.5^{\circ}$ to the right or an acceleration of $0.026 \mathrm{~g}$ to the left. This ambiguity is a real one and can lead to a number of illusions of erroneous spatial orientation. It is discussed in Ambiguity of Subjective Response to Acceleration, p. 1046. The ambiguity can be resolved only by using other information, such as semicircular canal signals, that indicates whether or not the subject has been rotated at a suprathreshold rate. The ambiguity can also be cleared up by reference to the expectation of the possible motions that might have been imposed, to information from nonvestibular cues, or by expectation based on voluntary movement.

\section{Static Orientation to Vertical}

Two principal methods have been employed in determining the ability of a subject to judge his orientation relative to the vertical in the absence of visual orienting cues. In the nulling method the subject, seated in a tilting chair, is normally displaced from the vertical and is permitted to return himself to what he feels is the erect position. The accuracy with which the subject can return to the vertical depends on the length of time he was left in the tilted position: long durations lead to adaptation or undershoot on the return. The speed at which he was originally tilted (higher speeds lead to higher accuracies) and the smoothness of the allowable return also influence the results. When applied to threshold measurements, subjects were typically able to judge correctly when they were tilted away from their assumed vertical $75 \%$ of the time if the tilt exceeded approximately $2.2^{\circ}$ (110). (To compare to linear acceleration thresholds, this corresponds to a lateral component of gravitation equal to $0.038 \mathrm{~g}$.)

Another widely used technique for judging the vertical from a tilted position is to have the subject align an illuminated line or rod to the judged vertical against a dark or homogeneous background. This use of the visual vertical allows the perceived angle of tilt to be tested without the complication of the dynamics of return associated with the nulling method. When the observer is seated in the upright position, this judgment can normally be made to within approximately $\pm 3^{\circ}$. Most importantly, this technique allows quanti- 
tative measurements of perceived tilt as a function of actual body tilt to be made at all body-tilt angles.

The estimation of body tilt with respect to the vertical, made by aligning a visible line to the perceived body axis without any other visual reference, might be thought to give the same indication of perceived tilt as the alignment of the line to the perceived vertical. Interestingly enough, the estimates of the body axis, although agreeing generally with the perceived vertical estimates for tilt angles up to $30^{\circ}-40^{\circ}$, continue to show an overestimation of body tilt for larger tilt angles. At $90^{\circ}$ of pitch or roll, for example, when underestimation of body tilt is maximum, the perception of the tilt angle of the body axis is always overestimated. Nearly all of the quantitative work on orientation perception, however, uses the indication of the visual vertical, and it is on this basis that this discussion is based.

For small tilt angles of the whole body about the $x$-axis, a truly vertical line appears tilted in the direction of body tilt, and in estimating the vertical with this line, many subjects tend to set it to a tilt opposite the direction of the body tilt. This overestimation is referred to as the Müller or E effect (123). As tilt angles increase beyond $30^{\circ}$, however, many subjects indicate a reversal in the direction of error and underestimate the amount of tilt. The line is then set tilted in the same direction as the actual body tilt. This is known as the Aubert or A effect (1). Not only does the principal error change as a function of the body angle, but the variability in making these estimates increases significantly for tilt angles above $30^{\circ}$. The range of individual differences for the $\mathrm{A}$ and $\mathrm{E}$ effects are shown in Figure 11 for 13 subjects whose apparent tilt angles are plotted. The increase in variability of the judgment of the vertical made from positions other than the upright has been associated with a dependence on saccular rather than utricular cues. As the tilt magnitude $\phi$ approaches $90^{\circ}$, the theoretical sensitivity of the utricular signals $g \cos \phi$ to changes in body angle approaches zero. The magnitude of the deviations from the true tilt angle may be varied on a centrifuge by increasing the strength of the specific force acting on the otoliths. Although ocular countertorsion occurs during the head tilt, it is certainly not the cause of the A effect. First of all, the ocular countertorsion would tend to reduce the angle of the true vertical relative to the vertical axis of the retina and consequently introduce errors in the opposite direction to the A effect, if no compensation was made
FIG. 11. Measurement of relative inclination of luminous line that subjects set to apparent vertical when they are tilted laterally. Range and median shown for 13 subjects. Overestimation of tilt at small angles is the $\mathrm{E}$ or Müller effect shown by some subjects. The underestimation A or Aubert effect at large angles is more pronounced. [Adapted from Bischoff (9), using data of Udo de Haes (157).]

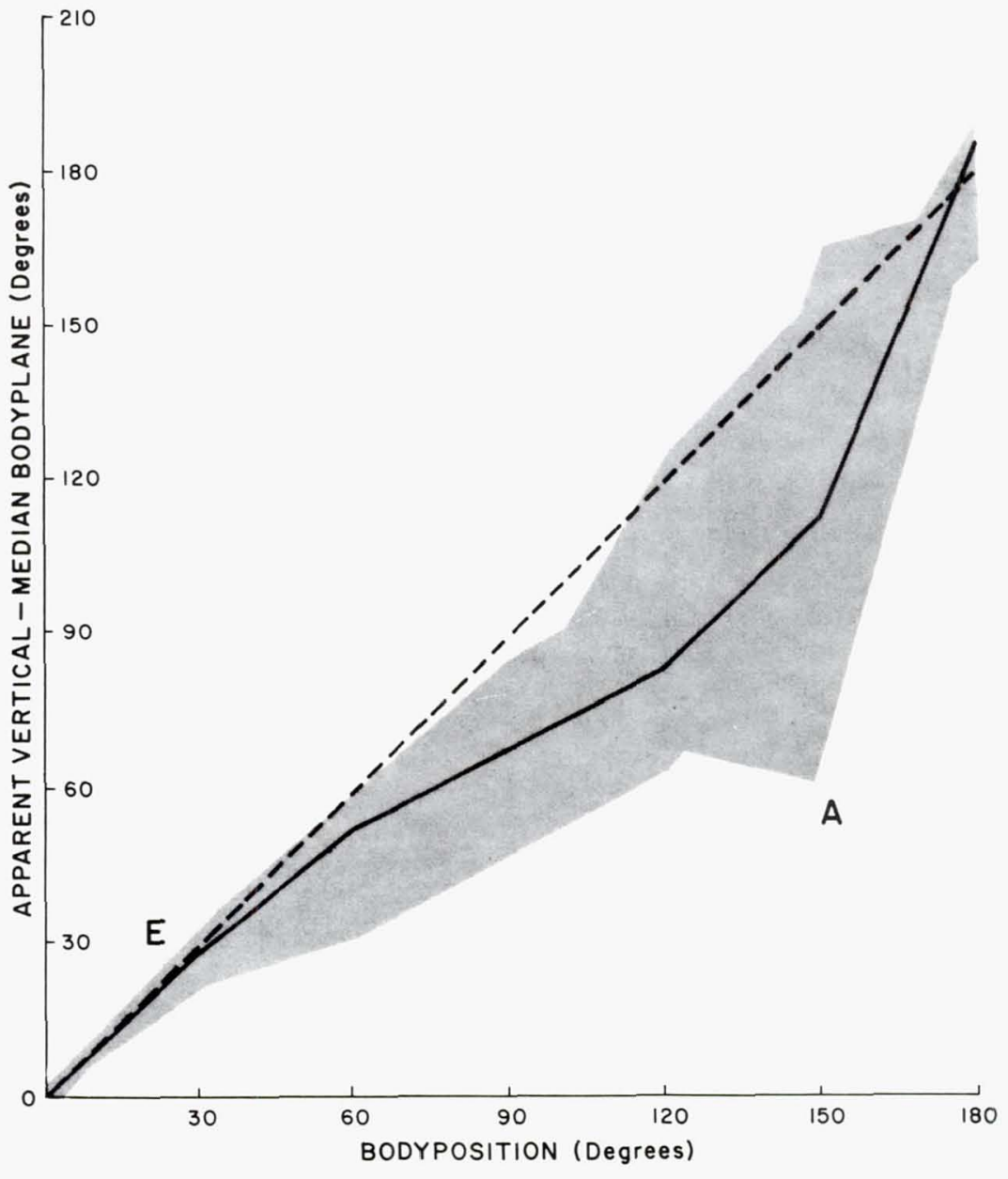


for eye movements. Fischer $(51,52)$ concluded, on the basis of direct measurements of ocular counterrolling and the A effect, that the phenomena were independent. Clearly, large angles of tilt underestimation up to $45^{\circ}$ could not be explained on the basis of ocular countertorsion, which normally does not exceed $6^{\circ}-$ $8^{\circ}$. Most conclusively, Fischer (53) demonstrated the existence of the A effect but no ocular counterrolling in a patient with no labyrinthine function.

The $\mathrm{E}$ effect for tilts up to $30^{\circ}$ is not limited to visual orientation of a line. Blindfolded subjects showed similar overestimation using a palpated rod to indicate the vertical (10). A similar technique was used in Skylab for judging orientation relative to the spacecraft (70). Auditory localization is also affected by the tilt in the same manner (153). Reviews of the various studies of the $\mathrm{A}$ and $\mathrm{E}$ effect in a 1-g field are given by Howard and Templeton (90), Guedry (76), and Schöne (141).

Similar consistent patterns of overestimation of small tilt angles and underestimation of large tilt angles also appear for pitch about the $y$-axis (141). There have been several attempts to explain the mechanisms of vestibular activity underlying the $\mathrm{A}$ and $\mathrm{E}$ effects. In particular, Schöne and his colleagues have supported the notion that the shear component of specific force lying in the plane of the utricular macula is primarily responsible for the illusion of tilt. At any given head pitch orientation $\theta$ and roll angle $\phi$ with respect to the vertical, the lateral and forward shear components in the plane of the utricle are given by the components

$$
\mathrm{f}_{y 0}=\cos \left(\theta-\theta_{\mathrm{u}}\right) \sin \phi
$$

and

$$
\mathrm{f}_{x \theta}=\sin \left(\theta-\theta_{\mathrm{u}}\right) \cos \phi
$$

$\theta_{\mathrm{u}}$ represents the pitch inclination of the major plane of the utricle with respect to the anatomical horizontal and is usually assumed in the human being to be pitched up or back by $25^{\circ}-30^{\circ}$ relative to the anatomic horizontal plane. Thus no forward shear component is present when the head is pitched forward $\theta_{\mathrm{u}}$, and all roll-tilt components, $f_{y}$, are maximized when the head is in the $\theta_{\mathrm{u}}$ pitch-forward position at the time of the roll. By setting the perceived tilt angle proportional to $f_{y}$ for roll and proportional to $f_{x}$ with a bias component of $\theta_{\mathrm{u}}$ for pitch, a rough approximation to the results of tilting in a static $1 \mathrm{~g}$ field is achieved. To fully understand the influence of compressive forces on the utricular macula and of the role played by the sacculus, however, it is necessary to examine the illusions of pitch taken under higher gravity loads. This is accomplished by placing subjects in gondolas at the end of a centrifuge arm and rotating at constant angular velocities so as to achieve a static gravitoinertial specific force vector greater than $1 \mathrm{~g}$. Perceived angle of tilt was measured when subjects were placed at various pitch and roll angles with respect to this resultant $\mathbf{g}$ vector. Different combinations of body tilt and resultant $\boldsymbol{g}$-vector magnitude could be used to independently vary the shear components in the plane of the utricle and the compressive components on the utricle. (The latter lie approximately in the planes of principal sensitivity of hair cells for the sacculus.) It became clear that the magnitude of the shear component in the lateral plane in the centrifuge experiments was not sufficient to predict the perceived tilt angle. Indicated lateral tilt at various specific force levels between $1 \mathrm{~g}$ and $2 g$ is shown in Figure 12. In all cases an increase in compressive force, keeping the utricular shear component fixed, led to an increased angle of tilt. A mere increase in utricular compressive component alone when no utricular shear component is present did not result in any change in perception of body pitch or roll $(36,140)$.

The perceived pitch or roll angle depends on the specific force perpendicular to the utricular plane as well as that in the plane. The utricular shear theory, although very useful for explaining perceived tilt, ocular countertorsion, and acceleration thresholds for small deviations from the upright position, generally is not valid. Several hypotheses have been considered to expand it to include the results of experiments performed at increased gravitation levels. One line of thinking maintains the primacy of utricular signals in determining orientation with respect to the vertical, but it includes nonlinearities. Among these nonlinearities is the possibility that the utricular shear signal saturates as the stimulus approaches $1 \mathrm{~g}$ in the plane

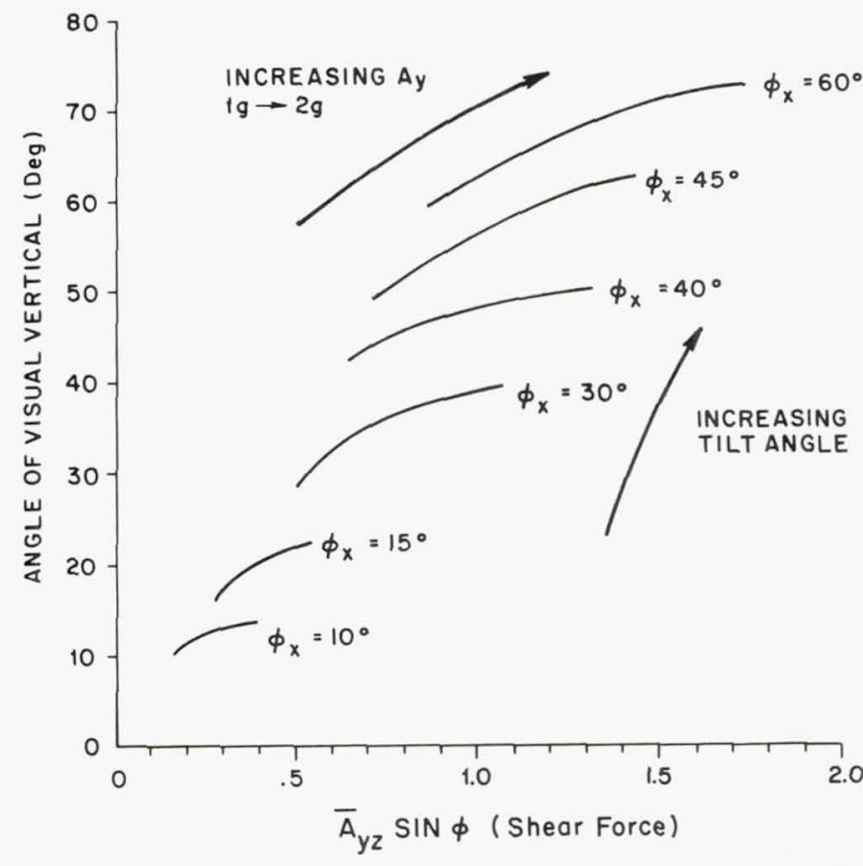

FIG. 12. Measurements of angle of a line set to apparent vertical, plotted against lateral component of specific force for various body tilt angles $(\phi)$ and $g$ levels. Apparent tilt varies according to shear force; it also increases with compressive force component. [Adapted from Correia et al. $(36,37)$.] 
of the utricles. Clearly, head tilts in the normal $1-g$ environment never produce sustained stimuli exceeding $1 \mathrm{~g}$, and one might argue that the transducer was not built to handle loads above $1 \mathrm{~g}$. Another explanation, based on physical mechanisms of displacement of the otolith membrane over the utricular macula, is simple and appealing. Correia et al. (37) pointed out that, once the hair cells were bent by a shear component of specific force, any subsequent compressive component would result in further bending of the hair cells, thus increasing the afferent signal and presumably increasing the perceived tilt. Benson and Barnes (5) developed this theory mathematically and showed that indeed single utricular afferent signals, as measured during head tilt and on centrifuges, could be explained on the basis of this combined loading. This notion is also consistent with measurements of ocular countertorsion that show a similar pattern of relatively large gain for tilt angles up to $60^{\circ}$ and much reduced gain for tilts beyond that in a 1-g field. Others have emphasized the role of somatosensory cues in accounting for the deviation of the visual vertical from the predictions of the shear hypothesis (102). All of these theories neglect the findings, now reasonably conclusively demonstrated, however, that stimulation of the sacculus plays a role in orientation in animals. Direct saccular stimulation results in eye deviations (57), and it has also been shown that the saccular signals respond to steady-state orientation about the vertical (50).

One general algorithm for prediction of overestimation and underestimation of pitch and roll at acceleration levels equal to and exceeding $1 \mathrm{~g}$ has been worked out by Ormsby and Young (132). The result of this theory is a simple diagram for predicting $\mathrm{A}$ and $\mathrm{E}$ effects in any gravity field, as shown in Figure 13. The heavy line pitched up by an angle of $\theta$ from the vertical when the head is in a normal erect position represents the dominant plane of the utricles. As usual, $\theta$ is taken to be $25^{\circ}-30^{\circ}$. The net gravitoinertial or specific force vector is indicated by f. It may have components perpendicular to the plane of the utricle, $\mathrm{f}_{z}$, in the lateral plane, $f_{y}$, or longitudinally in the plane of the utricle, $f_{x}$. The tilt is taken to be an underestimation (category A, Aubert illusion) when the compressive component, $\mathrm{f}_{z}$, is less than $1 \mathrm{~g} \times \cos \theta$, which corresponds to the compressive component present when the head is erect in a 1-g field. Similarly if the compressive force is greater than $g \cos \theta$, a Müller illusion or overestimation of tilt occurs. In category $\mathrm{N}$, when the compressive component of $\mathrm{f}_{z}$ is exactly equal to $g \cos \theta$, the veridical pitch or roll is presumed to be felt. The actual angle of perceived tilt that is predicted for any head orientation is calculated by the nonlinear transformation of $\mathrm{f}_{z}$ to $\tilde{\mathrm{f}}_{z}$, shown in Figure 14. The components of $f_{x}$ and $f_{y}$ are carried forward without nonlinearity and combined as a vector sum with $\tilde{\mathrm{f}}_{z}$ to produce the estimated vector representing the vertical. Figure 14 implies that information from the sacculus

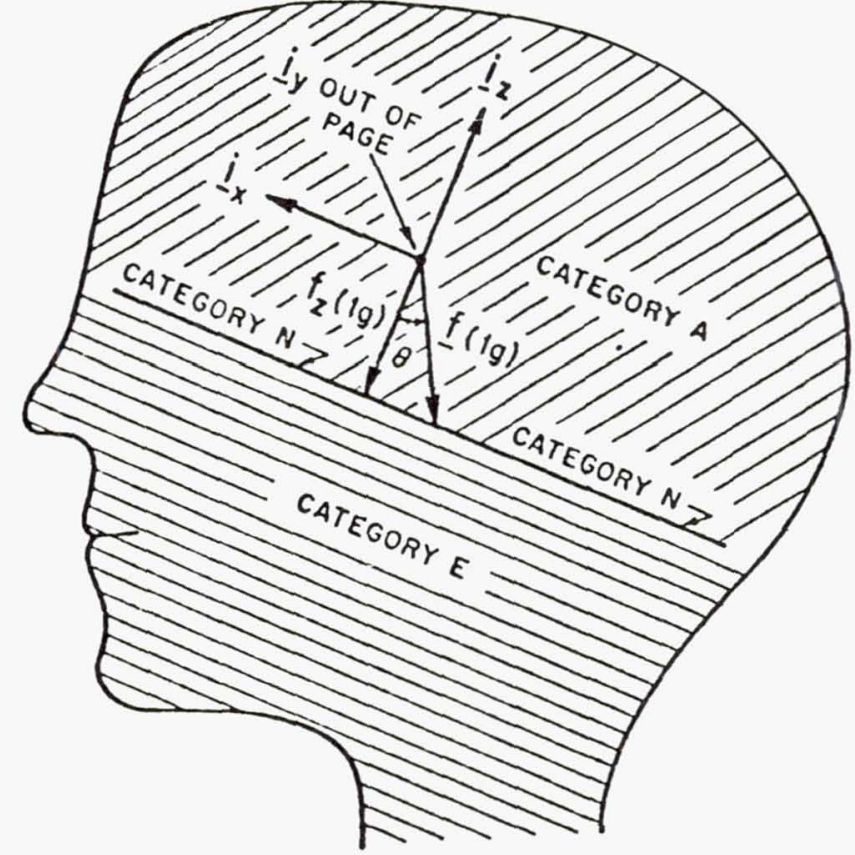

FIG. 13. Schematic representation of perceived pitch categories in various gravitational fields. The $z$-axis component of specific force determines whether actual pitch is underestimated (category A) or overestimated (category E). [From Ormsby and Young (132).]

is nonlinearly transformed, with a saturation at levels of saccular stimulation exceeding those that correspond to vertical accelerations greater than $1 \mathrm{~g}$, and with a slope of less than unity (range $0.6-1$ ) for acceleration components less than $1 \mathrm{~g}$. Although the results of a number of experiments are not predicted adequately by this theory, especially for head tilts greater than $90^{\circ}$ from the specific force vector, it does encompass many of the test results exceeding $1 \mathrm{~g}$, as indicated in Figure 15. According to the theory, the utricular shear for lateral tilt is dominant in determining orientation for small tilts away from the vertical, wherein the stimulation to the sacculus remains virtually constant. Saccular information, however, cannot be ignored for larger tilt angles or for higher acceleration levels. On the other hand, information from the sacculus is not transformed without error. It results in an ambiguity for vertical accelerations that produce net downward components of $\mathbf{f}$ greater than that which would be expected in a 1-g field. We refer again to this ambiguity in the interpretation of saccular information in treating the case of perception of orientation during vertical oscillations.

An alternative scheme for combining utricular and saccular information has been proposed by Schöne (141), and this scheme appears to match experimental data in a $1-g$ field at all angles. In that formulation, saccular signals merely switch the utilization of the utricular shear signal when the head is tilted more than $90^{\circ}$. 


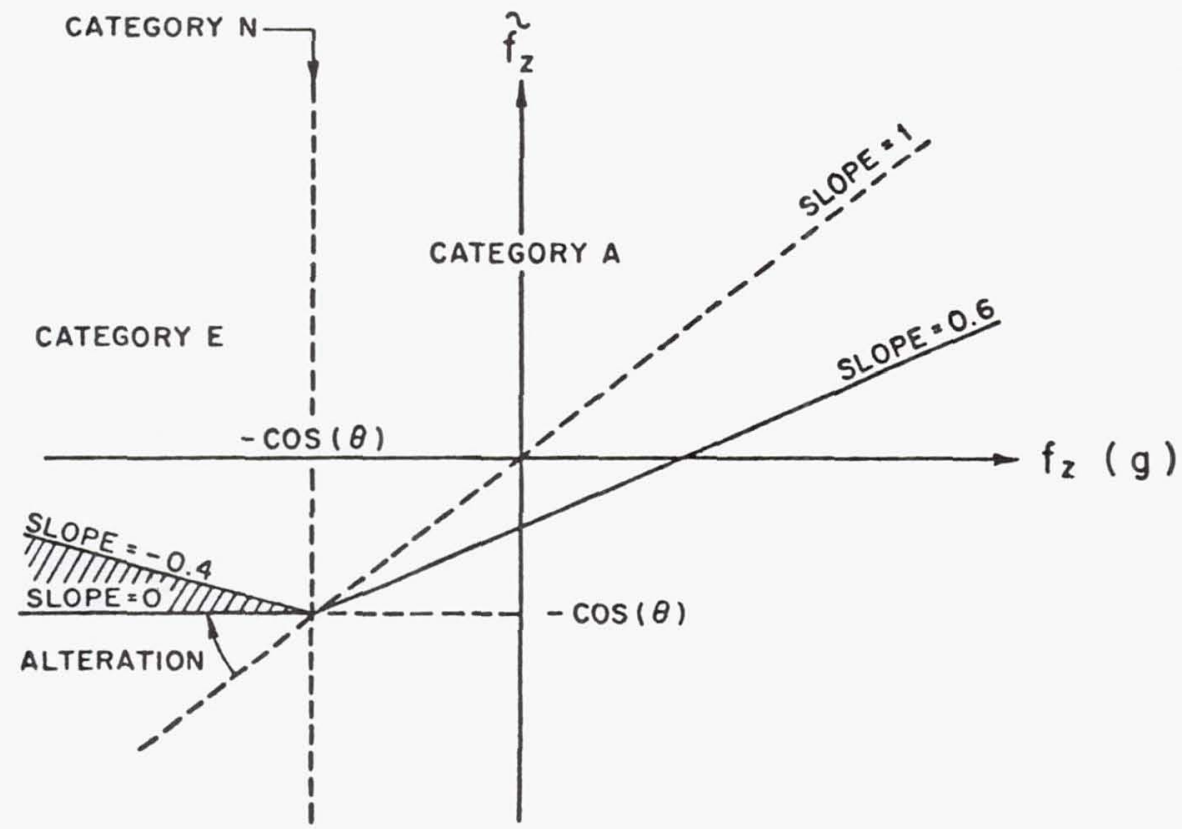

FIG. 14. Alteration of $z$-component of specific force to yield observed errors in perceived pitch. See text for explanation. [From Ormsby and Young (132).]

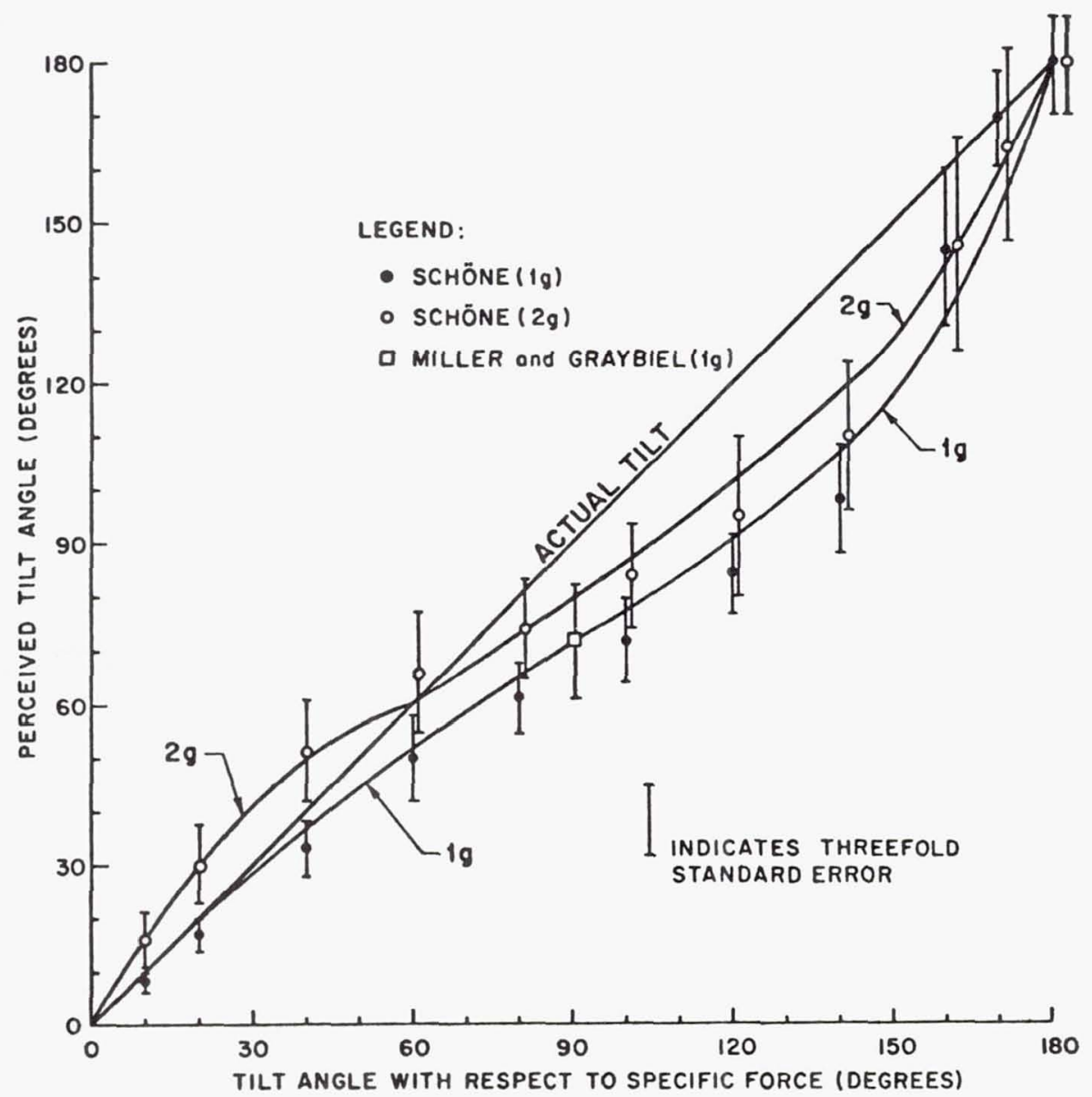

FIG. 15. Model predictions for perceived lateral tilt angle as function of actual tilt (as in Fig. 11) compared with data taken at $1 \mathrm{~g}$ and $2 \mathrm{~g}$. [From Ormsby and Young (132).] 
Contributions of Nonlabyrinthine

Sensors to Perception of Tilt

In all of the tilting experiments described above, subjects received consistent cues from the various sensory systems. Confirming cues emanated from the otolith organs, from surface and deep pressure tactile receptors, and occasionally from proprioceptive or kinesthetic receptors activated by the muscular effort required to maintain limb, head, or trunk postural stability in the tilt position. The preceding discussion emphasized the role of the shear component of specific force, especially on the utricular otolith, but also on the sacculus. Several techniques have been used to isolate the relative contributions of the labyrinthine and nonlabyrinthine (nonvisual) cues (76). Tactile and proprioceptive cues can be reduced, if not entirely eliminated, by various methods discussed in Reduced Somatosensory Cues, this page. Labyrinthine cues can be eliminated through the use of labyrinthine defective subjects in experiments. Static otolith cues are temporarily eliminated during free fall, in aircraft parabolic flight, or in spacecraft. Finally, somatosensory and proprioceptive cues can be augmented artificially by various techniques.

\section{Reduced Somatosensory Cues}

The tactile and proprioceptive cues afforded a normal subject may be reduced by using local anesthesia on the soles of the feet or the buttocks to eliminate support cues. Others have experimented with subjects who have suffered from spinal lesions, resulting in the interruption of afferent information from the parts of the body where support was provided. Most commonly, however, tactile cues were not eliminated but rather were spread out over a very large contact area, so that the peak pressure at any one part of the skin or over any one of the deep pressure sensors was kept relatively small. These latter methods either used form-fitting molds or were performed underwater. It should be clear that even in a neutral buoyancy underwater experiment, the average pressure on the lower side of the subject is greater than that on his upper side. The pressure differences are relatively small, however and may be imperceptible. When normal subjects are deprived of the usual somatosensory cues during tilt experiments, their average perception of tilt does not change noticeably, but the variance of their estimates of orientation increases markedly. Brown (20) carried out extensive experiments on the ability to perceive orientation with respect to the vertical when underwater and to right one's self with respect to the vertical. Errors in estimation compared with measurements taken with the normal-tilt chair support structure increased markedly, especially when the estimations were made with the head down or the face up. Especially large errors occurred when the tilt angle was more than $60^{\circ}$ from the erect position in pitch or roll. Schöne (139) found similar results in his underwater experiments wherein subjects indicated their orientation to the vertical by adjusting a line in the usual manner. With water immersion, subjects exhibited slightly greater $\mathrm{E}$ and $\mathrm{A}$ phenomena than when suspended from straps. The principal difference is the increased variance when somatosensory cues are reduced. Both Schöne and Brown found that the position with the head down led to the largest errors. When tests of alignment to the vertical were carried out on a centrifuge with normal subjects immersed in water to the neck, Graybiel et al. (71) found a relatively small reduction in perceived tilt relative to the same tasks carried out under dry conditions, as shown in Figure 16. Schock (138) also found that subjects attempting to set luminous rods to the vertical at various tilt angles underwater showed approximately twice the variability as they did when they were dry. Tactile cues do not normally introduce any major consistent bias in judgments that are primarily based on labyrinthine sensory information, particularly within $\pm 60^{\circ}$ of the erect orientation. They do, however, reduce the variability of such judgments.

\section{Labyrinthine Defective Subjects}

Unlike visual or auditory senses where the distal stimuli are easily removed by closing the eyes or blocking the ears, the vestibular sense organs of normal subjects cannot normally be shut off. Even in the absence of any linear or angular acceleration cues, the unmodulated resting discharge signals to the central nervous system that no measurable acceleration has

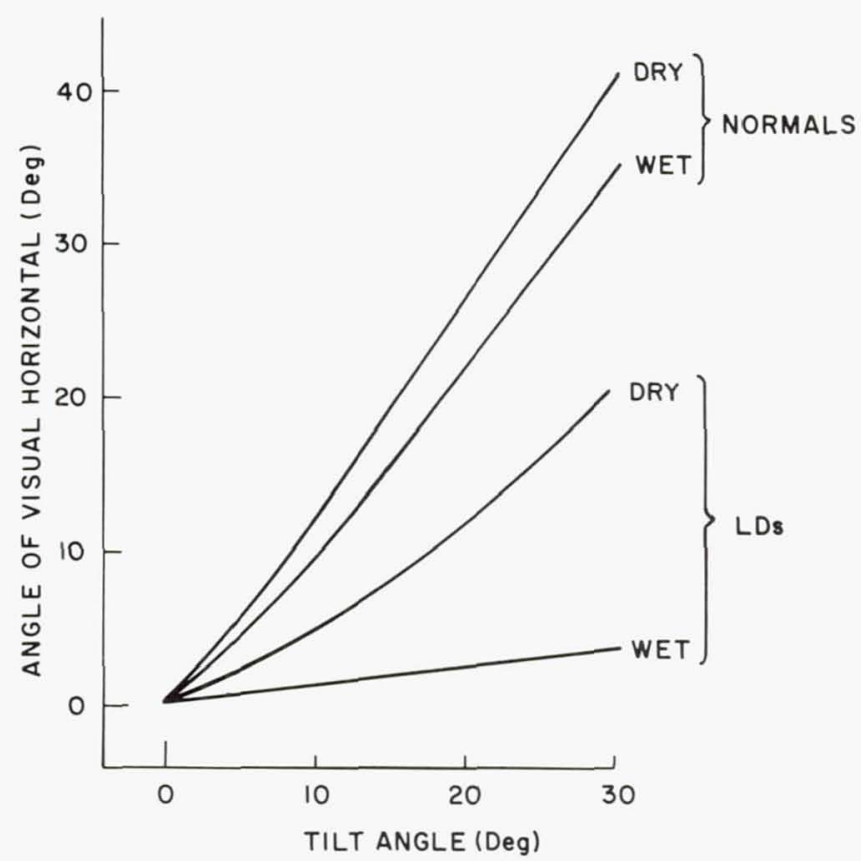

FIG. 16. Comparison between settings of line to perceived horizontal by normals and labyrinthine defectives (LDs) in air and when tactile cues were reduced by submersion in water. [Adapted from Graybiel (71).] 
taken place. The importance of this null signal is amply demonstrated by the generation of optokinetic afternystagmus (33) and by the production of motion sickness symptoms from visual stimuli alone in stationary subjects with functioning vestibular systems $(18,119)$. Consequently, to investigate the role of nonvestibular cues in determining orientation, and in particular for perceiving tilt, wide use has been made of subjects with bilateral loss of vestibular function. Loss of bilateral labyrinthine function is not uncommon among deaf subjects and may be the result of a number of disease entities or surgical intervention to treat severe auditory or vestibular problems. The absence of semicircular canal function is demonstrated by the absence of nystagmus during extreme caloric stimuli, and the absence of otolith function is demonstrated by a minimal ocular counterrolling response to head tilt.

Labyrinthine-defective (LD) subjects show a threshold to linear acceleration of the range of 15$26 \mathrm{~cm} / \mathrm{s}^{2}$ compared to thresholds in normals of $2-5$ $\mathrm{cm} / \mathrm{s}^{2}$. By way of contrast, removing substantial amounts of somatosensory input from normals by testing during water immersion raised thresholds to 4$7 \mathrm{~cm} / \mathrm{s}^{2}$, and thresholds of subjects with high spinal lesions interfering with somatosensory afferent feedback were also in the range of $4-8 \mathrm{~cm} / \mathrm{s}^{2}(164)$. The lower curves in Figure 16 show that the perceived tilt for $\mathrm{LDs}$ during rotation on a centrifuge is about onehalf that for the normal subject population. Furthermore the LD subjects, when deprived of their principal somatosensory information by water immersion, show a marked drop in the perceived tilt angle (71).

It is somewhat puzzling to note that the LD subjects consistently underestimate the angle of tilt with respect to the resultant vector on a centrifuge, whereas the normal subjects perform reasonably well, except for the $\mathrm{A}$ and $\mathrm{E}$ effects. On a tilt chair, however, where the direction of the specific force vector with respect to the head varies as much as it does for the centrifuge experiments, LD subjects are able to return themselves to the postural upright position with about the same accuracy as do normal subjects (30). Apparently the LD subjects, although much less accurate than normals in estimating the actual angle of tilt with respect to a force vector, are roughly equivalent in perception of tilt at one critically important anglethat which corresponds to maintenance of the head in the erect position. Not unexpectedly, when LD subjects are given a sufficient number of trials to practice returning themselves to the vertical from the tilt positions maintained for various periods of time, they do nearly as well as normals. Mean deviations for normals are of the order of $1.5^{\circ}$ compared to $2.5^{\circ}$ for LDs (143).

Experiments on perception of the vertical with tilted $\mathrm{LD}$ subjects afforded an opportunity to test the hypothesis that the $\mathrm{A}$ and $\mathrm{E}$ effects were associated with nonlinearities in the otolith organs. The $\mathrm{A}$ and $\mathrm{E}$ effects exist for $\mathrm{LD}$ subjects as well as normal subjects, making it clear that the otolith organs are not solely responsible for these systematic errors in perception of tilt. The magnitude of the effects, however, are far greater for the LD subjects than for the normals, and the transition from $\mathrm{E}$ to $\mathrm{A}$ phenomena occurs at smaller tilt angles with LDs than with normals (118).

Experiments on LD subjects, as well as those concerned with reduction of somatosensory cues for normal subjects, indicate that in the normal subject there exists a high degree of redundancy between somatosensory cues and otolith cues concerning orientation to the vertical. This redundancy leads, in the normal human being, to high repeatability in orientation and low thresholds for detection of acceleration or tilt. The illusions of underestimation of tilt are not uniquely attributable to the otolith organs or to the somatosensory system. In the absence of somatosensory cues, the variability in judgment increases. In the absence of labyrinthine cues, somatosensory cues are reasonably effective in the region of the upright head-erect position, but they fail for head angles more than $10^{\circ}-$ $20^{\circ}$ from the vertical.

\section{Amplified Somatosensory or Postural Cues}

Somatosensory signals may be distinguished from vestibular cues by independently altering the strength of the former. Relatively enhanced sensory cues can be provided during zero-gravity flight, when the otolith organs provide no indication of static orientation. A tactile indication of a local "down" reference can be established by pressing a subject down to his couch or with his feet against a surface using elastic tethers. Graybiel et al. (69) experimented with astronauts in the Gemini flights, and similar experiments were carried out by Graybiel et al. (70) on Skylab to determine the ability to maintain a sensation of body orientation with respect to the spacecraft in the absence of vestibular stimulation and visual cues. Settings of an apparent horizontal line with respect to the body axis were consistent and showed no obvious deviations, leading to the conclusion that the remaining tactile, proprioceptive, and kinesthetic cues, transmitted through the chair support, were sufficient to define this reference frame. Recent observations on the ability to voluntarily change one's sense of the direction of a local vertical during free fall has indicated that for many subjects tactile cues can be used for this purpose in the absence of otolith cues.

The voluntary muscular effort required to avoid falling can provide sensory cues that also contribute to judgment of orientation. Most of the tilt experiments discussed above were carried out with a seated subject. When similar experiments were performed with the subject standing, using tilts of up to $20^{\circ}$ to the right and left of the vertical, Clark and Graybiel (30) found that neither their LD subjects nor normal observers showed any significant errors in tilt perception, although the tilt angles were somewhat small. 
The notion that the muscular activity required to avoid falling influences perception of orientation is strengthened by experiments in which this activity is caused to be altered without head tilt. Uneven application of weights can produce asymmetrical forces on the head or body $(98,99,137)$, which is resisted by asymmetrical muscle tone. The muscle tension can be sensed by muscle spindles and by Golgi tendon organs. Wapner et al. $(166,167)$ showed that galvanic stimulation to the muscles to change muscular activity could also produce an asymmetrical tone. Each time the perception of body tilt was similarly influenced, just as though the muscular tone was necessary to avoid falling when tilted. Asymmetrical tactile cues have been used recently in aircraft flight simulators to provide the illusion of tilt or sustained acceleration (100). The application of asymmetrical tactile cues is achieved by differential elevation of various panels of a seat and back rest. The perception of increased magnitude rather than direction of the gravitoinertial acceleration is achieved by contouring the support so that all of the body's weight is taken on two highpressure points, the ischial tuberosities.

The perception of orientation with respect to the vertical in the normal subject is thus seen to be modified somewhat by manipulation of the somatosensory input. In the absence of such cues, judgments are frequently variable. The tendency to make great errors in judgment as one approaches the otolithic blind spot in the head-down position is particularly apparent. It is of interest to note that no such blind spot appears obvious in examination of the neurophysiological data. Representation of the changes in acceleration from any initial head position appears to be adequately present both in the first-order afferents from utricular and saccular units $(50,155)$ and in the units recorded in the vestibular nucleus. Certain directions of linear acceleration sensitivity do, however, appear to be preferentially represented at the level of the vestibular nucleus in the cat (41).

\section{DYNAMIC RESPONSE OF OTOLITH SYSTEM}

\section{Ambiguity of Subjective Response to Acceleration}

Horizontal-axis linear accelerations may be looked on as the vector sum of two orthogonal components of specific force, downward directed gravity and horizontal force opposite to acceleration. The total gravitoinertial vector, however, which is the vector sum of these two forces, swings through an arc just as a pendulum would during horizontal acceleration of its pivot. It is well known that the semicircular canals as well as the otolith organs respond to such rotating linear acceleration vectors (4) and that nystagmic eye movement and subjective rotation are elicited by these rotating stimuli. It is therefore not a simple matter to distinguish between the influence of the rotating vector and the influence of the time-varying horizontal component. Nor is it obvious whether the response that should be sought is one of perceived horizontal motion or perceived tilt with respect to the rotating vector. This difficulty can be overcome on earth by testing with dynamic linear accelerations along a vertical axis. In this case there is no rotation of the gravitoinertial vector, but all stimuli must take place about a 1-g bias level that may influence the results. The use of a centrifuge to build up horizontal components of acceleration presents additional problems. Either the subject is fixed in orientation with respect to the vertical, in which case the gravitoinertial vector rotates as in the case of the lateral oscillations, or he is in a pendulous swinging gondola that remains aligned with the gravitoinertial vector but which must tilt in order to do so, thereby stimulating the semicircular canals. Finally, transient linear accelerations normally take place beginning from a 1 -g bias. Only during brief exposures in the zero-gravity portion of parabolic flight or during linear acceleration experiments carried out within an orbiting spacecraft can this initial bias be removed.

When performing open-loop or magnitude estimation tasks, it is by no means clear what response should be sought from the subject. For horizontal accelerations, various investigators have attempted to elicit responses of subjective displacement or subjective acceleration. Most success has been achieved by asking subjects to respond with subjective linear velocity. In its simplest form this comes down to a judgment of "which way am I moving," which is particularly useful for determining the phase relationships between imposed and subjective velocity. The occurrence of the alternate subjective orientation response of pitching or rolling in response to horizontal acceleration depends to a great extent on mental set and the perceived degrees of freedom of the stimulating device. Sometimes after prolonged oscillation in the horizontal plane the initial perception of pure translation changes to one involving a strong perception of tilt during the peak acceleration phases at the extremes of the oscillation (133). As is true in studies of the rotation-sensing system, many of these difficulties in reporting techniques can be overcome by using the nulling method, in which the subject attempts to maintain himself stationary in space through closedloop control.

\section{Linear Acceleration Steps}

When a seated subject, deprived of visual cues, is accelerated in a horizontal direction along his $x, y$, or $z$ head axis, the time to correctly detect the acceleration rises as the acceleration magnitude is reduced for accelerations below approximately $0.05 \mathrm{~g}\left(0.5 \mathrm{~m} / \mathrm{s}^{2}\right)$. The data reported by Meiry (112) for accelerations along the $x$-axis are shown in Figure 17 . The model predictions indicated by the solid line follow from a theoretical model of the form 


$$
a_{x_{e}}\left(1+0.07 e^{-1.5 t}-1.07 e^{-0.1 t}\right)=\text { constant }
$$

where $a_{x_{e}}$ is the component of linear acceleration in the plane of the utricular macula and $t$ is the time required to detect the constant acceleration. Meiry assumed that the direction was based exclusively on shear forces applied to the utricle, and he neglected the possible use of saccular information. Thresholds were extrapolated from these data and taken to be $0.01 \mathrm{~g}$ for the supine position ( $z$-axis acceleration) and $0.006 \mathrm{~g}$ for the head-upright position. Meiry assumed that the absolute threshold, which would be reached for accelerations in the plane of the otolith with the head tilted forward $25^{\circ}-30^{\circ}$, would be approximately $0.005 \mathrm{~g}$.

A comparable set of experiments, measuring time to detect linear acceleration in the vertical axis and taking into account the $1-g$ bias, was carried out by Melvill-Jones and Young (117). The times to detect the onset of acceleration as a function of acceleration magnitude, shown in Figure 18, were remarkably similar for the vertical accelerations, indicating that the rotation of the linear acceleration vector and consequent stimulation of the semicircular canals was probably not an important factor in the response to horizontal acceleration. A convenient method of viewing these data is to recognize the roughly hyperbolic shape of the latency time-versus-acceleration curves and, allowing for the existence of some minimum reaction time $t_{\mathrm{r}}$, express the curves in the form

$$
t=B / a+t_{\mathrm{r}}
$$

where $t$ is the time to detect an acceleration step of magnitude $a$ and $B$ is the velocity constant, corresponding to the velocity that must be reached during brief linear accelerations before they are noticed. $B$ was found to be $21.6 \mathrm{~cm} / \mathrm{s}( \pm 2.6)$ and $22.6 \mathrm{~cm} / \mathrm{s}( \pm 1.3)$ for vertical and horizontal acceleration, respectively, both with the head erect. [Horizontal acceleration with subject supine raised the constant to $32.4 \mathrm{~cm} / \mathrm{s}$ $( \pm 2.0)$.] The calculated threshold for vertical acceler-

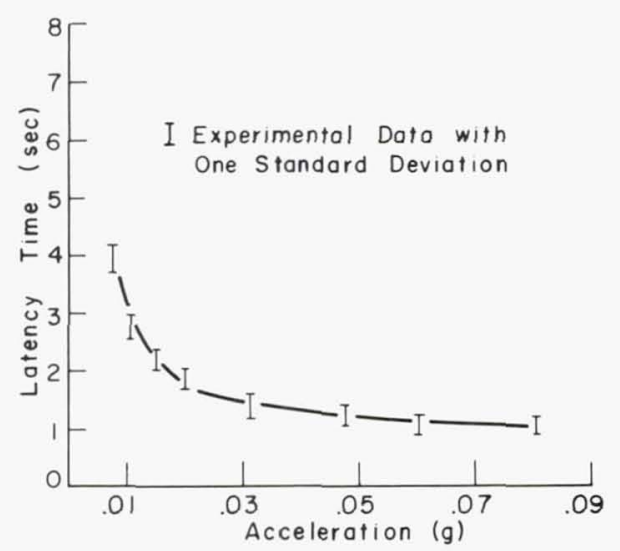

FIG. 17. Latency to detection of step of horizontal linear acceleration for subjects upright. See text for equation of model. [From Meiry (112).]

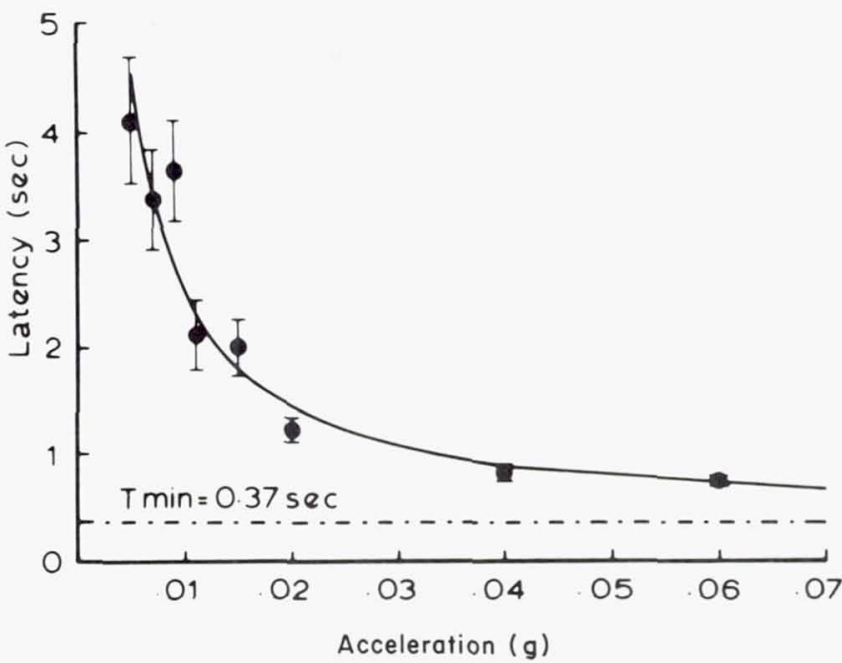

FIG. 18. Latency to detection of steps of vertical linear acceleration for subjects upright. Model is regression hyperbola for 8 subjects, yielding minimum response time of $0.37 \mathrm{~s}$ and velocity constant of $0.022 \mathrm{~g}$-s. [From Melvill-Jones and Young (117).]

ation, stimulating primarily saccular receptors, is nearly the same as that extrapolated for horizontal accelerations stimulating the utricular receptors. What is significantly different between the two axes, however, is the presence of a high degree of ambiguity and confusion in judging the direction of acceleration in vertical motion. Despite the brief latency times to detection of motion for the vertical, subjects were frequently wrong in their judgment of the direction in which they were moving. Frequently subjects who are given step or sinusoidal accelerations in the vertical direction are totally incapable of judging the direction of their current motion, although they are able to detect accelerations $(109,117)$. Because saccular units are presumed to have roughly the same sensitivity as utricular units to linear acceleration along their axes of polarization $(50,169)$, this threshold or sensitivity difference cannot be ascribed to the end organ, but a central nervous system integrative process must be investigated for an explanation. This difference in the treatment of saccular and utricular information is implicit in the static orientation model discussed in Static Orientation to Vertical, p. 1039.

\section{Sinusoidal Linear Acceleration}

Mach (106) reported that the thresholds to detection of vertical periodic motion on a seesaw-type device ranged between 10 and $12 \mathrm{~cm} / \mathrm{s}^{2}(0.01-0.012 \mathrm{~g})$ for himself and also for his technical assistant (87). A table of these thresholds, presented by Guedry (76), indicates a clustering of measures in the region of 0.005-0.01 $g$ for oscillations in the 2-to-9-s period range. One of the remarkable aspects of perception of linear oscillation is the normally unexpected occurrence of a substantial amount of phase lead at lower frequencies. Although there is not a complete overlap 
and agreement of data among investigators who have performed linear accelerations $(94,112,165)$, there is agreement that the phase lag between stimulus velocity and perceived velocity increases with frequency. The phase relation for horizontal linear acceleration along the $x$-axis is shown in Figure 19. Reliable subjective judgment of magnitude is much more difficult to obtain. Walsh was able to demonstrate the erroneous phase relationship to his subjects dramatically by having them open their eyes after having made a judgment of their direction of travel and thereby determine whether their perception was leading or lagging the actual motion.

The low-frequency phase lead and high-frequency phase lag implied by the data of Figure 19 are consistent with a linear transfer function relating the perception of linear velocity to the actual horizontal linear velocity given by

$$
\frac{\text { perceived velocity }}{\text { actual velocity }}=\frac{1.5(s+0.076)}{(s+0.19)(s+1.5)}
$$

The dominant time constant in this model $(5.3 \mathrm{~s})$ cannot be attributed to the mechanical events at the otolith organs by analogy to the relationship of rotation sensation to cupula return dynamics. The only direct measurements of otolith displacements during oscillation, taken by deVries (42) in the fish, indicate an extremely fast reacting system with dominant time constants of the order of $0.005 \mathrm{~s}$. Direct recording of first-order afferent units from the utricular and saccular maculae showed substantial sensitivity up to at least $2 \mathrm{~Hz}$. Fernández and Goldberg (50) fitted the frequency response data with a transfer function having a dominant first-order lag-time constant in the range of 5 to $30 \mathrm{~ms}$. (An additional long-adaptation time constant was highly variable among units.) $\mathrm{Ob}$ viously the long integration times shown in the perception data must be found other than at the end organ. Some evidence suggests that much of the processing of otolith signals takes place by the level of the vestibular nuclei, where substantial phase lags have been reported. The limited data reported by MelvillJones and Milsum (115) on frequency response of cat vestibular nuclei to linear sinusoidal oscillation indicates a substantial buildup of phase lag over the frequency range of $0.1-2.0 \mathrm{~Hz}$.

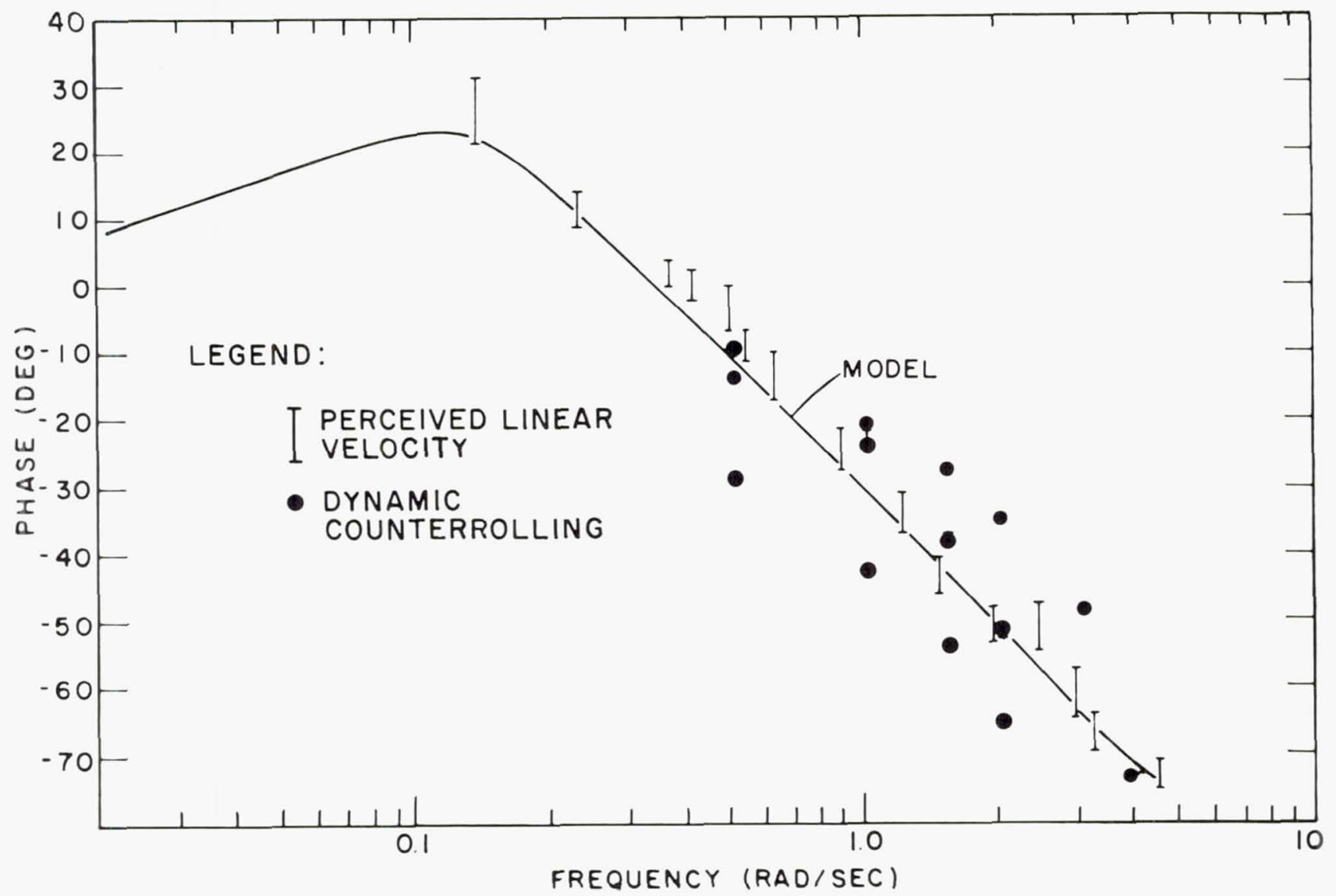

FIG. 19. Phase angle frequency response for perceived velocity vs. input velocity. Vertical lines showing \pm 1 SD. [Dynamic ocular counterrolling vs. lateral specific force, measured by Kellogg (96a). From Young and Meiry (177a).] 
COMBINED ROTATIONAL AND LINEAR ACCELERATIONS

\section{Consistent and Inconsistent Vestibular Signals}

Although most of the acceleration patterns discussed thus far involve stimulation of either the semicircular canals or the otoliths, the patterns of head movements encountered in daily life normally involve simultaneous stimulation of both types of labyrinthine organs. The simultaneous acceleration stimulation may be either consistent or conflicting. Of course all of the sensory cues are, in a certain sense, consistent if one takes into account the physical stimulation situation and the dynamic response of the sensors. The term consistent, however, may be restricted to the simple situations in which the interpretation of perceived motion may be reached by considering current semicircular canal output signals to represent head angular velocity and current otolith signals to represent direction of the specific force vector. A simple example of consistent cues is a rapid rolling motion of the head from the upright position $90^{\circ}$ toward the right shoulder. If the movement is completed in less than $2 \mathrm{~s}$ or so, the vertical semicircular canals indicate approximately the correct instantaneous head-roll angle velocity, which can be integrated centrally to calculate the change in head-roll angle. This change in head angle of $90^{\circ}$ is consistent with the short-term measurements made by the utricular and saccular otolith organs, indicating that the specific force vector now lies along the head $y$-axis, having rotated $90^{\circ}$ from the head $z$-axis. Minor deviations from accurate determination of orientation associated with the A effect or with inaccuracies in canal transduction are ignored. The consistent interpretation based on canal and otolith signals is the veridical head roll. If this action were the result of active muscle contraction rather than passive rotation, the perception would furthermore be consistent with the efferent copy determination of the result of muscular contractions. Finally, if this action were performed with eyes open in a stationary environment, the perception would agree with that based on visual cues (relative rotation of the retinal image), taking into account the small effects of ocular torsion.

Simple examples of conflicting cue situations are more difficult to find, although one may be a common experience to the reader. During constant forward, $x$, acceleration lasting more than a few seconds, such as is experienced during the takeoff roll of an airplane, the specific force vector rotates rapidly from the vertical to a position lying in back of the true vertical. The passenger or pilot is forced back into his seat, pressure cues on the back increase, and the utricular otolith organ detects a component of specific force in the $-x$ direction. The new specific force vector lies between the $x$-axis and the $-x$-axis, as shown in Figure

\section{AIRCRAFT MOTION AND ATTITUDE}
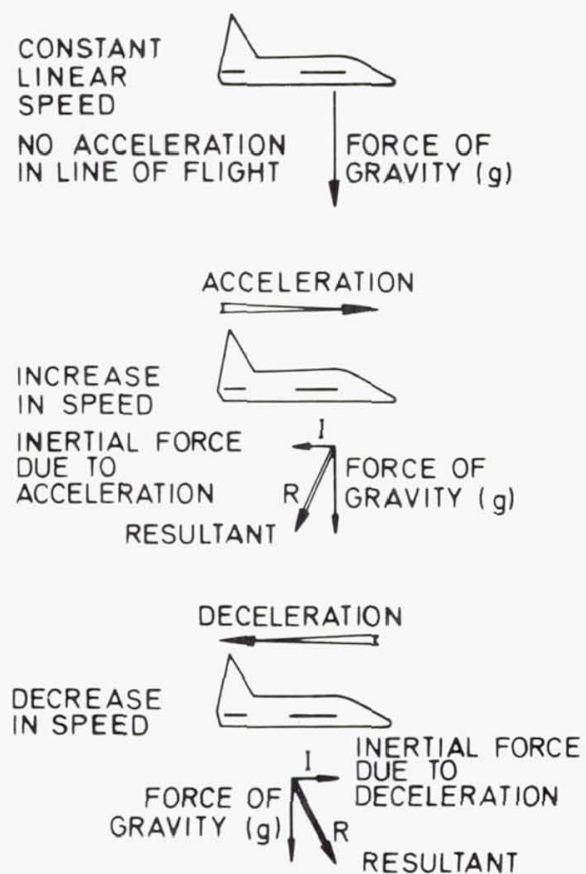

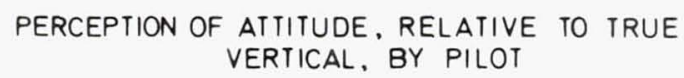

OF HIMSELF
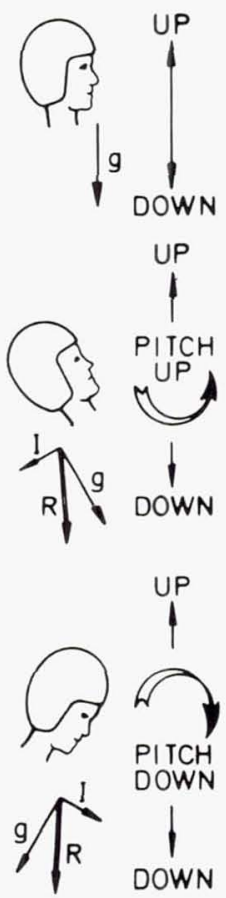

OF HIS AIRCRAFT
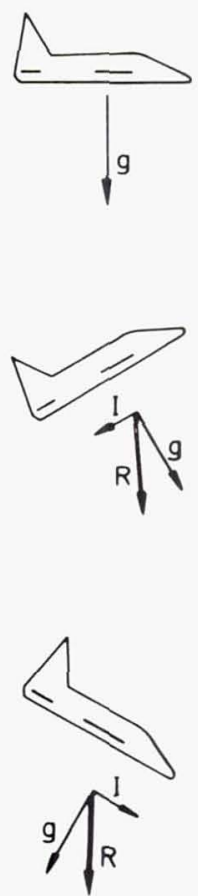

FIG. 20. Illustration of perception of pitch during constant acceleration. [From Benson (4a).] 
20. All of the linear acceleration mechanisms therefore indicate a situation consistent with a change from the upright to a pitched-back orientation. The vertical semicircular canals, however, having received no angular acceleration input (neglecting the direct effects of linear acceleration on the cupulae for the moment), signal no change in pitch angular velocity, which was assumed to be zero at the outset. Consequently there is a conflict between the simple interpretation of linear acceleration cues as a pitch up and the lack of confirmation by semicircular canal signals. What in fact normally happens, in the absence of visual signals or a mental set dictating the contrary, is an initial perception of forward acceleration without any change in pitch angle, which only gradually shifts to the replacement of the acceleration sensation with one of steadystate pitching up. The generally accepted explanation is that, with the passage of sufficient time compared with the dominant time constants of the semicircular canals, the lack of a confirming semicircular canal signal is given less weight in the conflict with otolith signals, and that in the steady state the otolith signals dominate in perceiving static orientation with respect to the specific force vector. This pitching-up illusion is of practical importance when the accelerations are large. On catapult-assisted takeoffs from an aircraft carrier deck, the illusion of excessive pitching can be quite severe, even without consistent semicircular canal cues. The unfortunate pilot, who believes that he has been pitched up excessively during the launch and reacts by pushing the nose downward, risks an abrupt crash into the water. The following sections illustrate some of the better known examples of conflicting vestibular cues and their resolutions.

\section{Centripetal Acceleration}

Consider a subject who is riding in a fixed chair at the end of a centrifuge arm when the centrifuge rapidly spins up to a consistent angular velocity. If the subject is facing "into the wind" so that his $y$-axis lies along the centrifuge arm, the centripetal acceleration is directed inward along his $-y$-axis (for counterclockwise centrifuge rotation) and the centrifugal force is directed out along his $+y$-axis. The net specific force vector rotates from the true vertical outward, as illustrated in Figure 21. The specific force vector rotates outward by an angle $\theta$, given by $\theta=\tan ^{-1}\left(\omega^{2} / g\right)$. The subject senses the initial acceleration to angular velocity $\omega$ about his $z$-axis with his horizontal semicircular canals. After several canal time constants, the sensation of rotation about the $z$-axis decays to zero. The rotation of the specific force vector creates a situation similar to that of the pitch illusion during acceleration, discussed in Ambiguity of Subjective Response to Acceleration, p. 1046. Although the otolith cues and the tactile cues are all consistent with the simple explanations of a subject rolling outward by an angle $\theta$ with respect to the vertical, they are not confirmed by signals from the vertical semicircular canals. Furthermore, if the subject had been actually rolling while yawing about his $z$-axis, cross-coupled angular acceleration would also have introduced a sensation of pitch, which is similarly lacking. In any event the perception that the vertical is aligned with the specific force vector lags dramatically behind the actual rotation of the specific force vector (67), as indicated in Figure 21. Presumably the delay in adopting the changing direction of the specific force vector as the vertical is tied to the conflict with the semicircular canal signals, and it awaits the passage of several time constants of the canals before these conflicting signals can be ignored.

Another situation in which the semicircular canal and otolith cues conflict is a coordinated turn in an aircraft. In this most common of all aircraft maneuvers, as illustrated in Figure 22, the aircraft is maneuvered from straight and level flight into a constant rate of turn $\omega$ by rolling to a bank angle $\phi$. A typical pattern of rolling into and out of a sustained constantrate coordinated turn of radius $r$ is shown in the figure. The specific force vector, $\mathbf{f}$, which is the vector sum of gravity $g$ and negative acceleration $r \omega^{2}$, remains
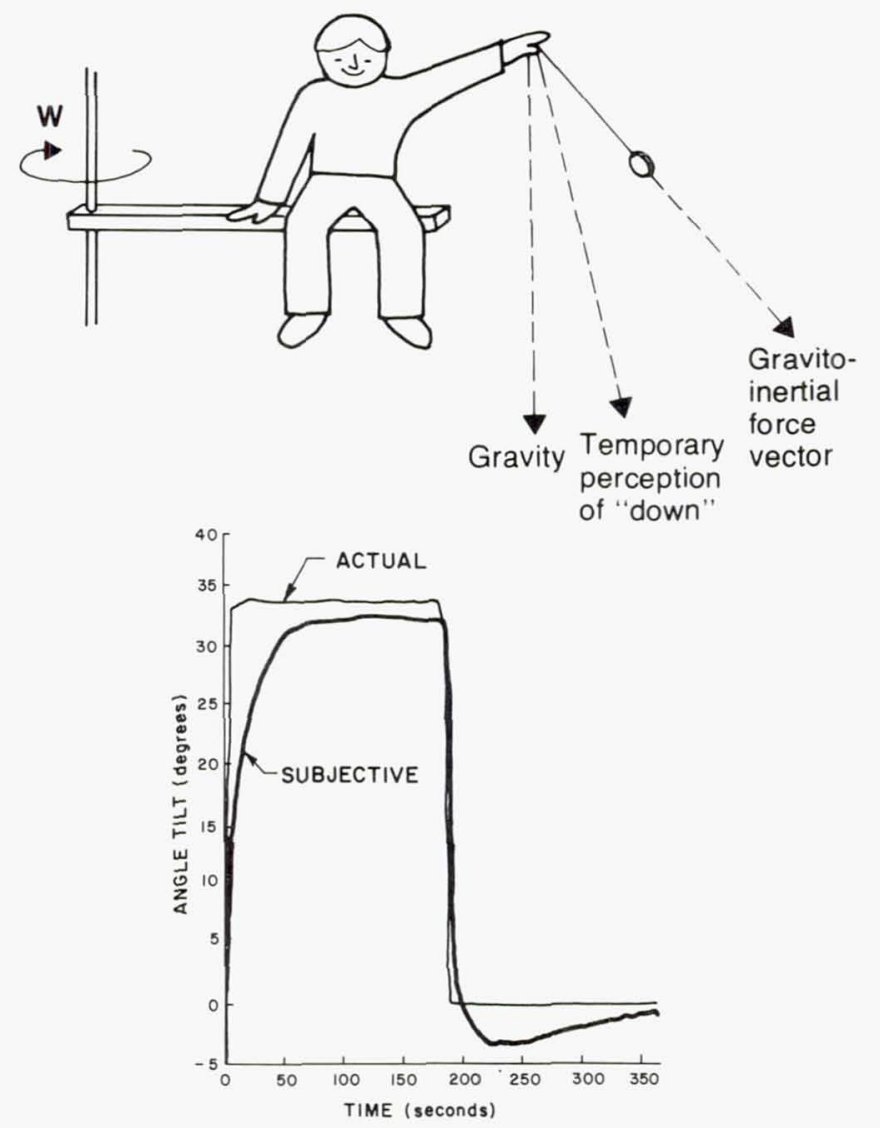

FIG. 21. Shift of direction of specific force vector during constant velocity centrifuge rotation produces slow shift in direction of estimated vertical or horizontal. [Adapted from Young (175a) and Graybiel and Brown (67).] 
AIRCRAFT ORIENTATION
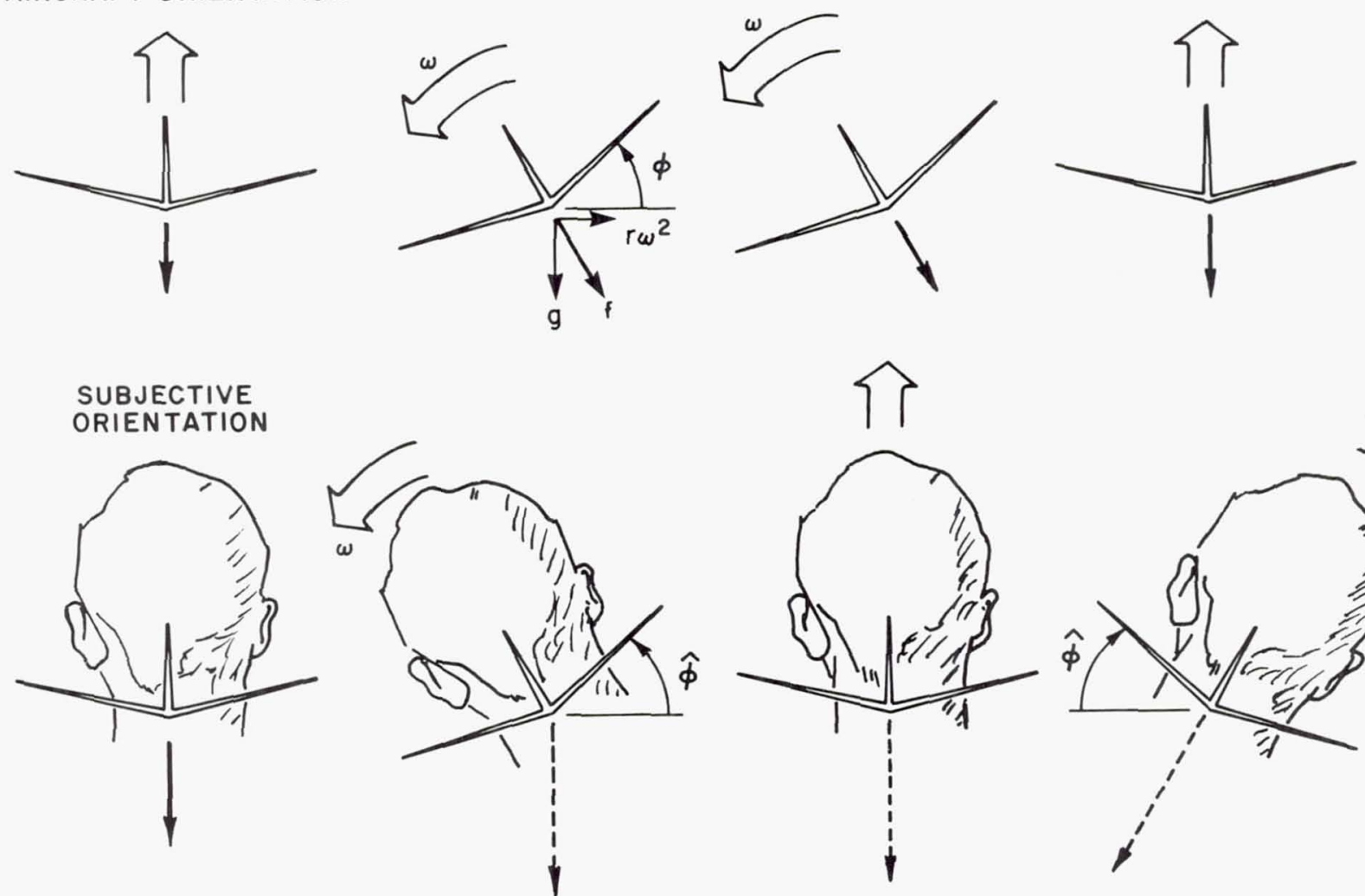

aligned with the $z$-axis of the aircraft and of the pilot and passengers during the coordinated turn. As a consequence, a conflict develops between semicircular canal and otolith cues about the roll angle. The vertical semicircular canals, sensitive to the brief roll rate, correctly indicate the roll into the turn during initiation of the roll rate, and they indicate the existence of zero roll rate during the constant turn. The otolith system and the other graviceptor sensors, however, do not detect any change in the orientation of the specific force vector with respect to the body; the specific force vector always points directly down through the bottom of the aircraft. The only change the graviceptor sensors experience is a small increase in the magnitude of the specific force vector from $g$ to $g\left(1+r \omega^{2} / g\right)$. Because the graviceptive cues do not confirm the existence of a steady-state roll angle, the perception of this roll is quickly washed out, unless confirmed by visual cues, instruments, or previous knowledge of the maneuver. Furthermore, the turning rate $\omega$ is usually constant and becomes subthreshold. The perceived roll is indicated schematically by $\hat{\phi}$ in Figure 22 . On rolling out of the coordinated turn and back into straight and level flight at the end of the maneuver, the reverse process takes place. Beginning with the perceived roll angle of zero, the subject interprets the transient semicircular canal cues as a roll in the opposite direction of the initial turn (to the right in Fig.
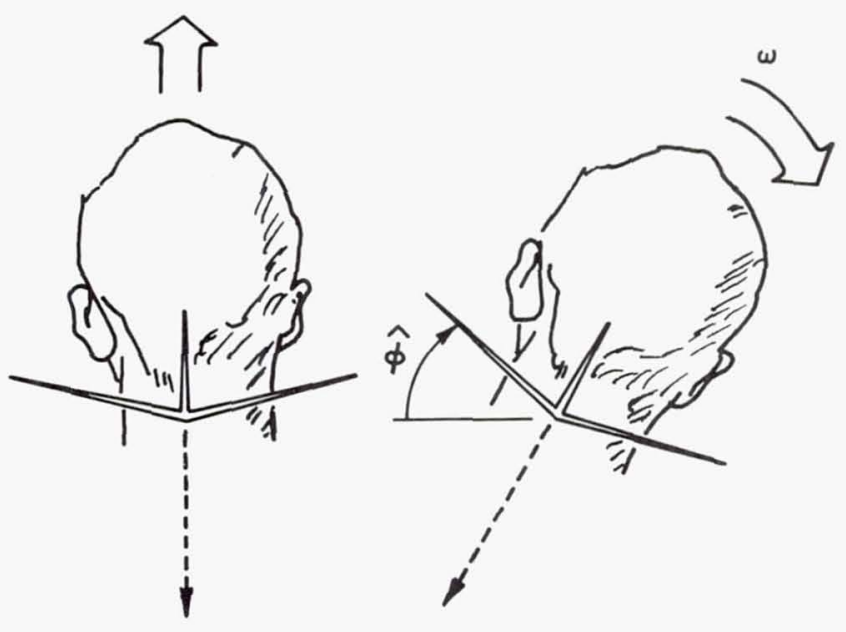

FIG. 22. Coordinated turn leading to error of spatial orientation.

$22)$. Because the direction of the specific force vector still remains aligned with the subject, however, this sensation of rolling in the opposite direction during straight and level flight is normally not maintained. The situation is further complicated by the postrotatory sensation in yaw (discussed in Rotation in Dark, p. 1029), in which the horizontal semicircular canals signal a postrotatory turn to the right, consistent with the illusion of roll to the right. The coordinated turn is a simple but well-documented case of practical importance in which the conflict exists between semicircular canal and otolith cues. It is resolved normally by short-term and transient reliance on semicircular canal signals (which are relatively high-frequency transducers of roll angle changes) and long-term or steady-state reliance on otolith and other graviceptive cues. It is of such practical importance in aviation safety that it serves as a fundamental case for teaching pilots during instrument flight to believe their instruments. In the presence of visual fields indicating the veridical motion of the aircraft, of course, the conflict is easily resolved correctly.

\section{Cross-Coupled Angular Accelerations; Coriolis Illusion}

A common perceptual illusion that causes almost as much confusion in its discussion as in its experience is 
that of cross-coupling due to simultaneous rotation about more than one axis. Both the perception of spatial rotation and the discomfort associated with such head movements are explainable on the basis of signals processed by the semicircular canals and otoliths. Consider, first, a subject who is rotating at constant angular velocity counterclockwise (left to right) about his $z$-axis vertical, which is the normal position for spinning with the head erect. Shortly after beginning this rotation, the horizontal semicircular canals, having integrated the acceleration necessary to reach this velocity and not yet having had sufficient time for the cupulae to return, correctly indicate the magnitude and direction of his angular velocity. Let us call this velocity $\omega_{z}$. Now permit the head to be tilted rapidly $90^{\circ}$ to the left shoulder, assuming for simplicity a constant-roll angular velocity of $\omega_{x}$ for this tilt. During the tilting motion, the ongoing vertical-axis angularvelocity vector $\omega_{z}$ is rotated in head-fixed axes from the yaw axis to the pitch axis at an angular velocity of $\omega_{x}$. This rotation, represented as an angular acceleration about the head roll axis, is a real angular acceleration of magnitude $\omega_{z} \omega_{x}$, directed along the $y$-axis. It would be detected by any angular accelerometer sensitive to accelerations about the $y$-axis, including the vertical semicircular canals, which detect pitch. Consequently the vertical canals detect and transmit the transitory acceleration leading to a perception of pitch forward during the period of head tilting. (This transient acceleration disappears when the head has reached its new position.) As a result of this crosscoupled acceleration, the ongoing angular velocity about the vertical axis $\omega_{z}$ has been removed as a stimulus from the yaw semicircular canals and appears as a stimulus to the vertical pitch semicircular canals. The horizontal canals signal a deceleration to zero, and the vertical canals signal an acceleration to the actual vertical axis angular velocity, which is now about the head pitch, $x$, axis. All of these phenomena represent the veridical situation and, to this point in the discussion, would cause no conflicting signals or disorientation. In fact, such a head movement carried out just after initial acceleration causes minimal disorientation and no discomfort (77).

The more interesting and more disturbing aspects of cross-coupled angular acceleration occur when the head tilt is made after a prolonged period of constant angular velocity. If we assume that the angular velocity of spin about the vertical axis discussed in the paragraph above has been going on for $30 \mathrm{~s}$ or more prior to the head tilt, then it is apparent that prior to the head tilt the yaw semicircular canal cupula would have returned to its rest position and the perception of angular velocity about the $z$-axis would also have returned to zero, as illustrated in the left portion of Figure 23. Assuming that the roll rotation is a passive one in the dark, the subject would be taken completely by surprise by the resulting yaw and pitch semicircular canal signals and the perceptions that ensue from a head tilt to the left shoulder during this ongoing spin. The transient cross-coupled angular acceleration indicating a pitch-back sensation during head tilt occurs, just as in the case discussed above. In this situation, however, because the subject would presumably have an indication of ongoing $z$-axis angular velocity, the sensation would come as a complete surprise and does, in fact, cause alarm and confusion. The pitch axis semicircular canals are accelerated from null to $\omega_{z}$ and indicate to the subject a sudden and unexpected acceleration about the vertical axis $\omega_{s}$, as above. The horizontal semicircular canals, having been in their rest condition prior to the head tilt, indicate a sudden deceleration when the ongoing vertical axis counterclockwise velocity is removed from them by the head tilt. The horizontal semicircular canal cupulae are deflected by this deceleration, and they indicate an opposite (postrotatory) velocity, this time in the clockwise direction about the head yaw axis, represented by the yaw component of $\omega_{\mathrm{s}}$. Consequently, following the cross coupling associated with the head tilt (which would be detected even by perfect angular-velocity detectors), this head movement leaves as an unpleasant residue a sensation of spinning clockwise about the yaw axis, which is now in the horizontal plane. This aspect of the cross coupling, which is certainly unexpected and unpleasant, depends on the imperfect integration in the semicircular canals. To make matters worse, there is a discordance between the signals indicated by the semicircular canals and those indicated by the otoliths. For the first case discussed above, in which the head movement is made immediately at the initiation of the vertical axis spin, the otoliths signal the veridical head orientation with respect to vertical, and the only conflict comes about during the head tilt itself, when otolith signals do not indicate any pitch. For the second case, in which the horizontal semicircular canal deceleration results in a perception of clockwise rotation about the yaw axis horizontal, there is an absence of any otolith timevarying signal to confirm this sense. Stated more simply, the horizontal semicircular canals signal a continuous rotation, but the utricular and saccular maculae indicate that the head has not, in fact, succeeded in changing its orientation with respect to gravity. The illusions of pitch and roll, when considered in detail, are relatively complex $(77,113)$. The sensations of discomfort and frequently of motion sickness resulting from these cross-coupled angular accelerations are most likely attributable to the conflict between semicircular canals and nonconfirming otolith cues rather than to the unexpected cross-coupled angular acceleration signal acting on the canals themselves. Some support for this theory stems from the results of the cross-coupled angular-acceleration experiments carried out during weightlessness on the Skylab space mission. Although the initial tests were not carried out early during the weightless period, so the possibility of generalized motion sickness habituation exists, it 

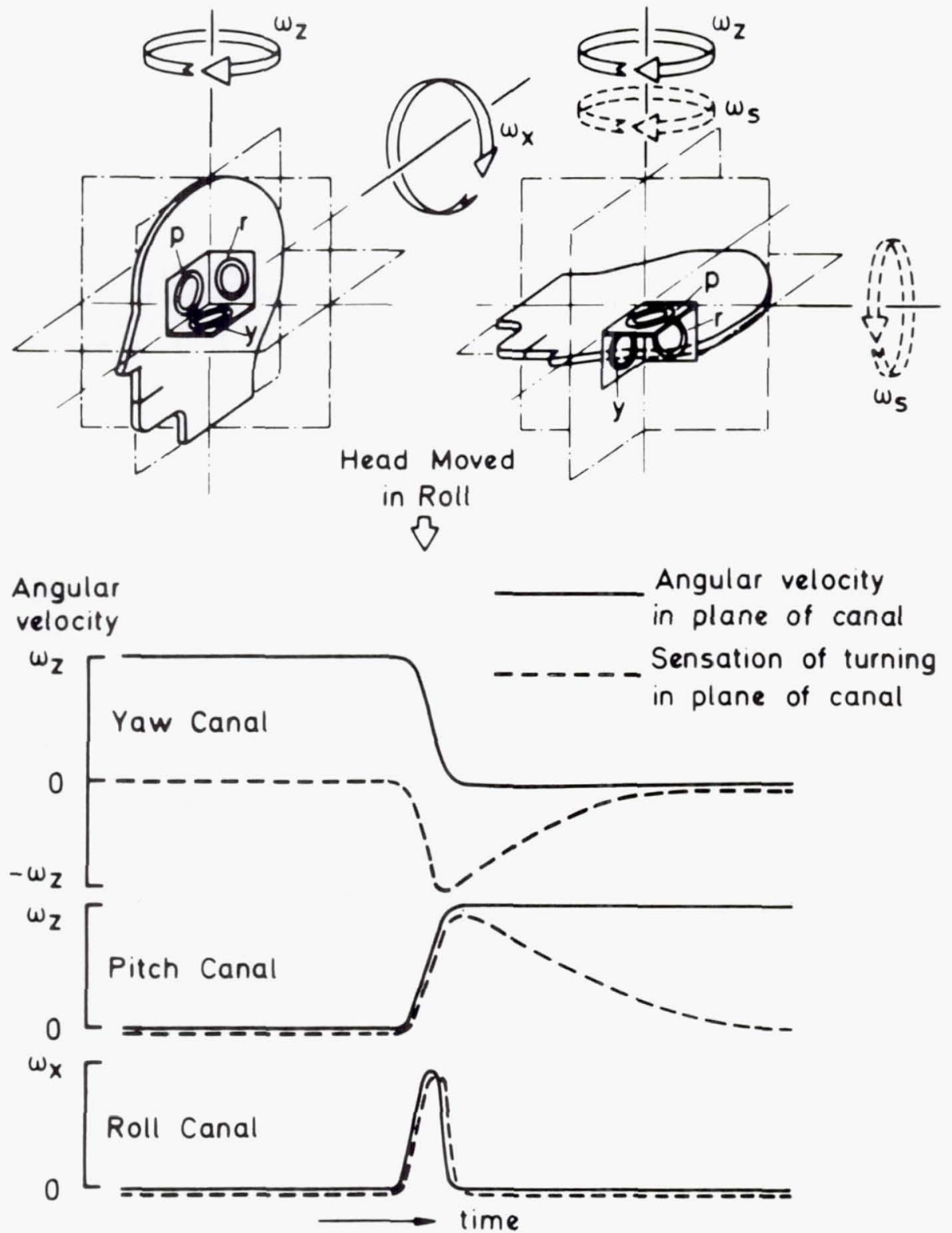

FIG. 23. Illustration of cross-coupled stimulation. Rolling head movement, $\omega_{x}$, during sustained yaw rotation, $\omega_{z}$, leads to erroneous transient perception of yaw and pitch velocity. [From Benson (4a).]

was found that subjects were able to make essentially unlimited numbers of cross-coupled angular-acceleration head movements in the weightless condition without reported motion sickness symptoms, such as they reported under similar conditions on the ground. In weightlessness, of course, no steady-state otolith conflict existed to support or to contradict the semicircular canal cues following head movements (70).

\section{Rotation About Off-Vertical Axes}

It was indicated in Semicircular Canals, p. 1025, and Otolith Organs, p. 1026, that the principal role of the semicircular canals in perception of orientation is detecting angular velocity, whereas that of the otolith organs is detecting either static orientation with respect to the vertical or linear acceleration. Changes in the otolith signal that are interpreted as changing orientation with respect to the vertical may also be used to infer angular velocity, in which case their information is comparable to that normally expected from the semicircular canals. The simple rolling of the head to the shoulder is an elementary example of this multiple-cue integration. A more complex example, which does not normally occur in daily life, involves sustained constant-angular-velocity rotation about the longitudinal $z$-axis when this axis is off vertical or not aligned with gravity. Consider for simplicity the 
situation in which the longitudinal axis is aligned with the earth horizontal and rotation is carried out at constant angular velocity about the yaw axis. This bizarre orientation is sometimes referred to as "barbecue spit stimulation." On initiation of the rotation, of course, angular-velocity signals from the vertical (roll) semicircular canals are in agreement with the changing orientation of the head with respect to gravity, indicated by the otoliths. After 15-30 s of constant-angular-velocity rotation, however, the semicircular canal signals have decayed toward zero, except for a small remaining gravity-sensitive modulation. Nevertheless the perception of the actual continuous rotation remains during the stimulation $(4,6$, $35,76)$. Clearly this is a case where there is a conflict between semicircular canal information, which decays to its rest state and does not indicate any rotation (although strictly speaking it supports any constantvelocity hypothesis) and dynamic otolith information, which is consistent with continuous rotation. Otolith information in these instances appears to dominate. Simultaneous recordings of nystagmic eye movements (4) and the results of experiments with selected blockage of semicircular canal or otolith signals support the view that otolith information is processed to indicate the sustained rotation. A closely related conflict occurs when the subject is brought to a sudden stop after barbecue spit rotation, and the previous rotation axis remains in its off-vertical orientation. The postrotatory sensation signals from the semicircular canals, opposite in direction to the per-rotatory motion, are in conflict with the signals from the otolith organs that indicate static orientation with respect to the vertical. No significant postrotatory turning sensation occurs, and the postrotatory nystagmus is much weaker and briefer than for rotation about a vertical axis. Evidently when otolith and tactile cues or previous knowledge of the stimulus situation indicate to the subject that any motion will be about an off-vertical axis, confirming graviceptor cues are necessary and sufficient to produce the perception of rotation or inhibit it, despite transient semicircular canal signals. Similar perception of constant angular velocity of pitching about the pitch axis occurs when the subject is rotated at constant angular velocity with the $y$-axis horizontal. [One report, however, based on a single subject rotated at constant angular velocity with tactile cues minimized by underwater immersion, contradicted this observation and described a perception of circular or elliptical counterrotation as if on a ferris wheel (151)]. Guedry (75) demonstrated that an intact vestibular apparatus is required both for the development of the continuous rotation sensation during off-vertical rotation and for the associated nystagmus. The absence of these responses in subjects lacking a normal vestibular apparatus (75) is strong evidence for vestibular contributions to the sustained rotation sensation. The possibility of direct linear acceleration effects on the semicircular canals is still open, although the bulk of evidence supports the central integration of otolith and canal signals (4).

VISUAL EFFECTS ON PERCEIVED ORIENTATION-MODELS

\section{Static Visual Orientation}

Straight lines in a visual field play an important role in defining a subject's perception of the vertical or horizontal. When a subject looks at a tilted room, or one that is made to appear tilted through the use of prisms or mirrors, he associates the vertical with the line tilted toward the direction of the walls of the room. If the subject is placed in such a room, his postural reactions are consequently to align himself parallel to the walls, and his reactions are consequently destabilizing. The tendency to associate trees, walls, and the like with the vertical and apparent horizons with the horizontal is at the basis of many well-known illusions, such as the "streams that run uphill" or the "haunted swing" (172). Serious disorientation of pilots can result from the illusion that the tops of a tilted cloud bank or the line of street lights near the edge of a town represent the horizon $(60,61)$. The extent to which visual scene straight lines influence the perception of vertical or horizontal is highly variable among individuals. The field dependence or field independence is generally measured by use of the "rod and frame" test (171), in which the subject is required to align an illuminated rod to the perceived vertical when the rod is surrounded by a large square frame which may be tilted from an upright position. Although individual differences in field dependence have been associated with sex, intelligence, and personality traits (170), the basis for the field dependence is not fully understood.

Meaningful elements in static visual scenes are not essential to influence the perception of the vertical. Any distinct axes, which may be contained in a pattern of parallel stripes or on a grid, produce a tendency to align the closest vertical or horizontal with the axis $(60,142)$.

\section{Moving Visual Fields}

Uniform motion of a large part of the visual field can induce a false sensation or illusion of self-motion. A common example occurs to the passenger in an automobile or train, himself stationary, when the neighboring vehicle begins to move slowly. The common illusion is that one's own vehicle is moving in the opposite direction. Such visually induced self-motion, in which the relative movement of the visual field is perceived as attributable partially or entirely to movement of the observer through the field, is known as vection. It is used to advantage in the induction of a sense of motion in wide-field-of-view flight simulators, large-screen movies, and in various amusement park 
exhibits or rides in which the sensation of movement is created or heightened by visual surround motion.

When a wide visual field suddenly begins to rotate about a stationary subject, the perception of veridicalfield motion is generally replaced by a perception that the visual field slows, often to a stop, and is replaced by subjective rotation (circularvection) beginning typically $2-5 \mathrm{~s}$ after stimulus initiation for rotations about a vertical axis. The strength of the self-motion sensation builds gradually over a period of $3-10 \mathrm{~s}$, rising in a roughly exponential fashion to some steady level. For field velocities less than $50 \mathrm{deg} / \mathrm{s}$, the circularvection may be complete or saturated, in which case the visual field is perceived as stationary in space. If the onset of the visual-field motion is gradual, at acceleration levels comparable to the acceleration thresholds of the semicircular canals (on the order of $0.2 \mathrm{deg} / \mathrm{s}$ ), then the latency to onset of circularvection may be negligible, and all of the perceived motion may be interpreted as self-motion. A number of factors contribute to the strength of vection and its rapidity of onset.

The peripheral visual field is of primary importance for the development of vection (72). Although the moving visual field generally induces nystagmic eye movements beating in the direction of the field, such eye movements are not required for generating selfmotion. In fact, oppositely beating nystagmus may be generated by a central moving field without interfering with the peripheral field-generated vection $(19,54)$. Although the specific content of the moving visual field is not particularly important for inducing vection, the spatial frequency of the field and in particular the rate at which contrast borders move over the retina is important in generating vection effects (84). Moving objects that appear to be in the background of the visual scene are considerably more effective in generating vection than are moving objects in the foreground.

The subject's view of his own body can only enhance vection, whereas visible fixed objects in the background interfere with it (19). The relationships between the characteristics of the visual field and the dynamics of vection are covered in several recent reviews $(44,45,86,175)$. Although the quality of the visually induced motion is in almost all respects identical to the motion perception resulting from true body motion with its attendant vestibular and proprioceptive cues, there exists at least one important difference. Vection exhibits relatively frequent and not yet explained dropouts, in which the sensation of self-motion is suddenly reduced to zero and replaced by the veridical sensation of visual-field motion. During these dropouts, although the sensation of velocity changes, there is no simultaneous sensation of sudden deceleration. The relationship of these dropouts to eye movements and conflicting vestibular signals is still being explored. Furthermore, during exposure to continuous constant-velocity visual scenes, the visually induced motion sensation gradually adapts so that the perceived self-velocity during sustained constant-field velocity stimulus is gradually reduced (8). A common example of linearvection adaptation is the underestimation of automobile speed after prolonged highspeed driving.

In addition to the continuous perceived self-motion velocity, a paradoxical self-motion sensation is created when viewing a field that rotates about the axis of gaze along an earth horizontal axis. This illusion, termed visually induced tilt (46), may easily be observed by staring at the axis of a disc rotating with its axle horizontal. The initial illusion resembles that of circularvection, a perception that the field slows to a stop and that the viewer begins to fall in the opposite direction. This self-rotation sensation, however, does not result in a continuous and ever-increasing perception of roll or pitch angle, which would lead to a perception of inversion and beyond. Instead, a static perception of tilt ensues in the direction opposite to that of visual-field rotation, leading to inappropriate postural responses and to the setting of the perceived vertical in the direction of field motion. The paradoxical self-motion perception (continuous rotation without increasing angular deviation) is apparently created by the conflict of visual signals with the otolith sensory information that indicates no tilt about the horizontal axis.

\section{Static Visual-Vestibular Interaction}

The retinal signal (proximal input) from a fixed external visual field (distal input) varies with head tilt. This variation of proximal signal must in some manner be compensated for head and eye movements in order to have vertical objects continue to appear vertical despite head tilt. As discussed in Static Orientation to Vertical, p. 1039, this compensation is not perfect but results in systematic underestimation or overestimation of tilt (the Aubert and Müller effects). There clearly exists some mechanism by which varying retinal images lead to perceptions of the same orientation of the visual distal signal. As illustrated schematically by Bischoff (9) in Figure 24, the interference variable associated with head tilt is presumed to be compensated. Although there is some measure of external compensation consisting of ocular counterrolling, in which the torsional movements of the eye are in the direction necessary to maintain ocular stability despite head movements, these are vestigial in man, and the tonic steady-state ocular torsion for head movements up to $60^{\circ}$ is normally less than $10 \%$ of that required for full compensation. Consequently one must look to some internal process for the compensation of the tilted retinal signal as a result of varying head roll angles. The most straightforward of the internal compensation mechanisms, building on early outflow ideas of Helmholtz (85), Bühler (23), Kardos (95, 96), and others, was later formulated by Von Holst and Mittel- 


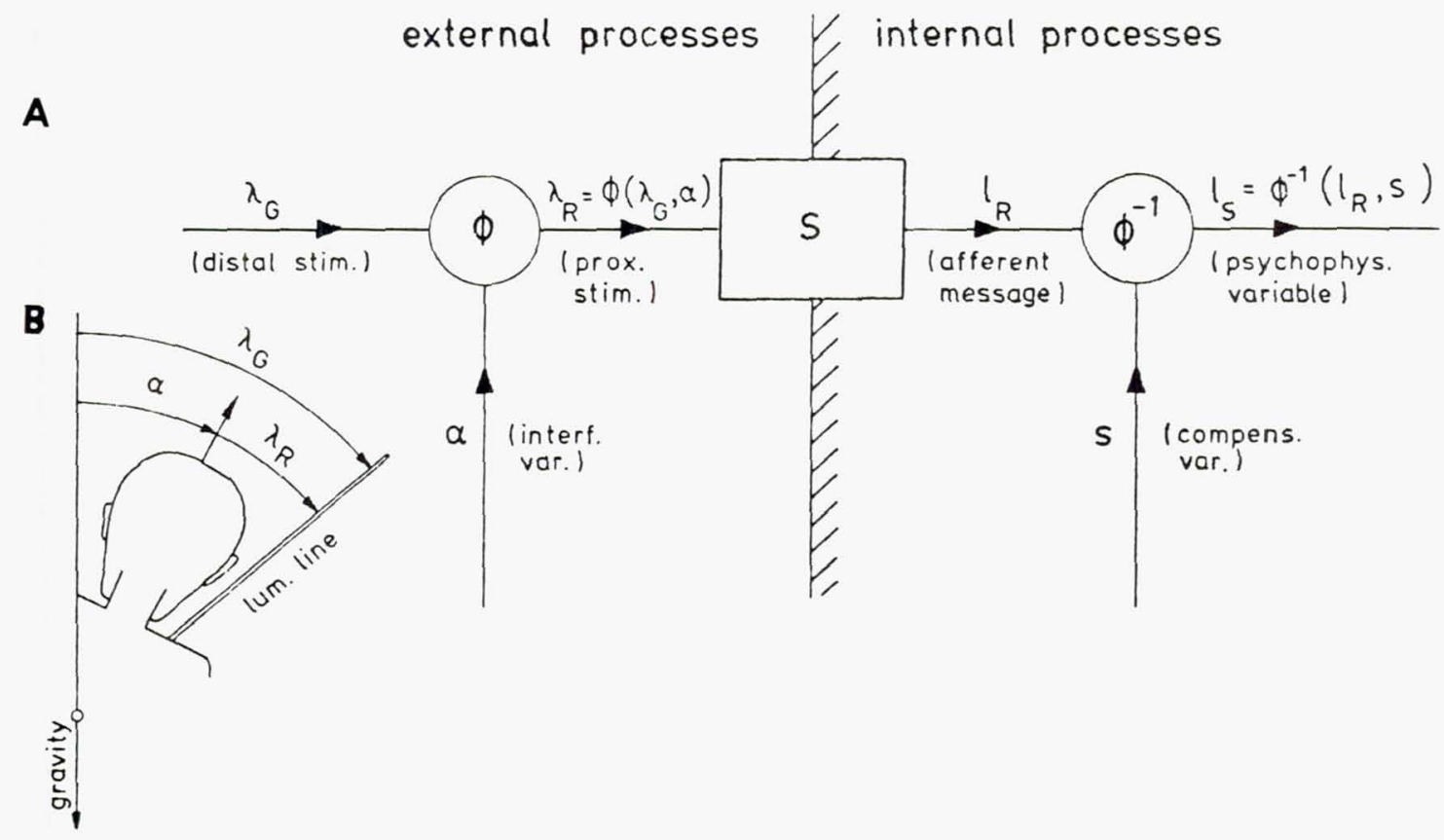

FIG. 24. Schematic representation of compensation principle for effect of head tilt on retinal image or proximal stimulus. $\phi$, Interference function; $\phi^{-1}$, compensatory function; S, mapping function of sensory channel. [Adapted from Bischoff (9).]

staedt (162) and others as the reafference principle. When applied to the correction or reinterpretation of the visual image by nonvisual signals, indicating head tilt, this compensation is referred to by Bischoff as heterocompensation. Simply stated, vestibular, kinesthetic, or tactual cues assess the amount of head tilt and apply the appropriate internal correction signal to compensate the tilted visual scene and allow it to be perceived as upright. To complicate the story, however, are the observations that the orientation of the visual field itself can lead to changed perception of the vertical, which in turn can affect the perception of the orientation of that scene. Specifically, the indication of the vertical is influenced by the orientation of a background visual scene containing axes that may be thought of as vertical or horizontal, by head orientation, and by the interaction between these two stimuli. Based on a complex set of experiments involving rotations of both the head and the visual field about a vertical axis, Bischoff and Scheerer (10) arrive at evidence to support the feedback-autocompensation theory for the manner in which the compensation signal is developed based on optical as well as vestibular inputs. The perceptual output itself is involved in the filtering process that goes into determining the sense of vertical, and this axis rotation then feeds back to participate in interpretation of the proximal image coming from the retina. Because visual as well as vestibular cues influence the perception of self-orientation and consequently the interpretation of the orientation of visual scenes, as well as postural reactions, the interaction of static visual and vestibular signals provides an interesting challenge to mechanistic modeling. Referring primarily to evidence from the static orientation of fish in response to varying angles of incident light, Von Holst and Mittelstaedt (162) expressed the relative influences of the two sensory inputs on orientation in terms of variable weighting functions. They (162) formally developed a trigonometric addition theory for this linear weighting notion. Even in fish, as Von Holst pointed out, the relative spatial orientation weighting given to optic input depends on how interesting or meaningful the object is in the visual scene.

In dealing with human spatial orientation problems, especially in the context of disorientation and motion sickness, many investigators stress the importance of intersensory conflict and its resolution. Young (174) represented the visual-vestibular interaction problem in humans as a flow chart (shown in Fig. 25) in which visual and vestibular responses to orientation were weighted linearly provided they compared reasonably well, reminiscent of the ideas of Von Holst for fish orientation. When the deviation between the visual and the vestibular signals exceeded some tolerable level, however, the content and quality of the visual information was examined to determine whether or not it was "compelling." A compelling visual field, consisting of recognizable objects and strong orientation cues, would tend to then be accepted as representing the external vertical reference, whereas uncertainty in accepting the visual field would lead to greater reliance on the otolith signals. If, however, neither were compelling (as for example in the head- 


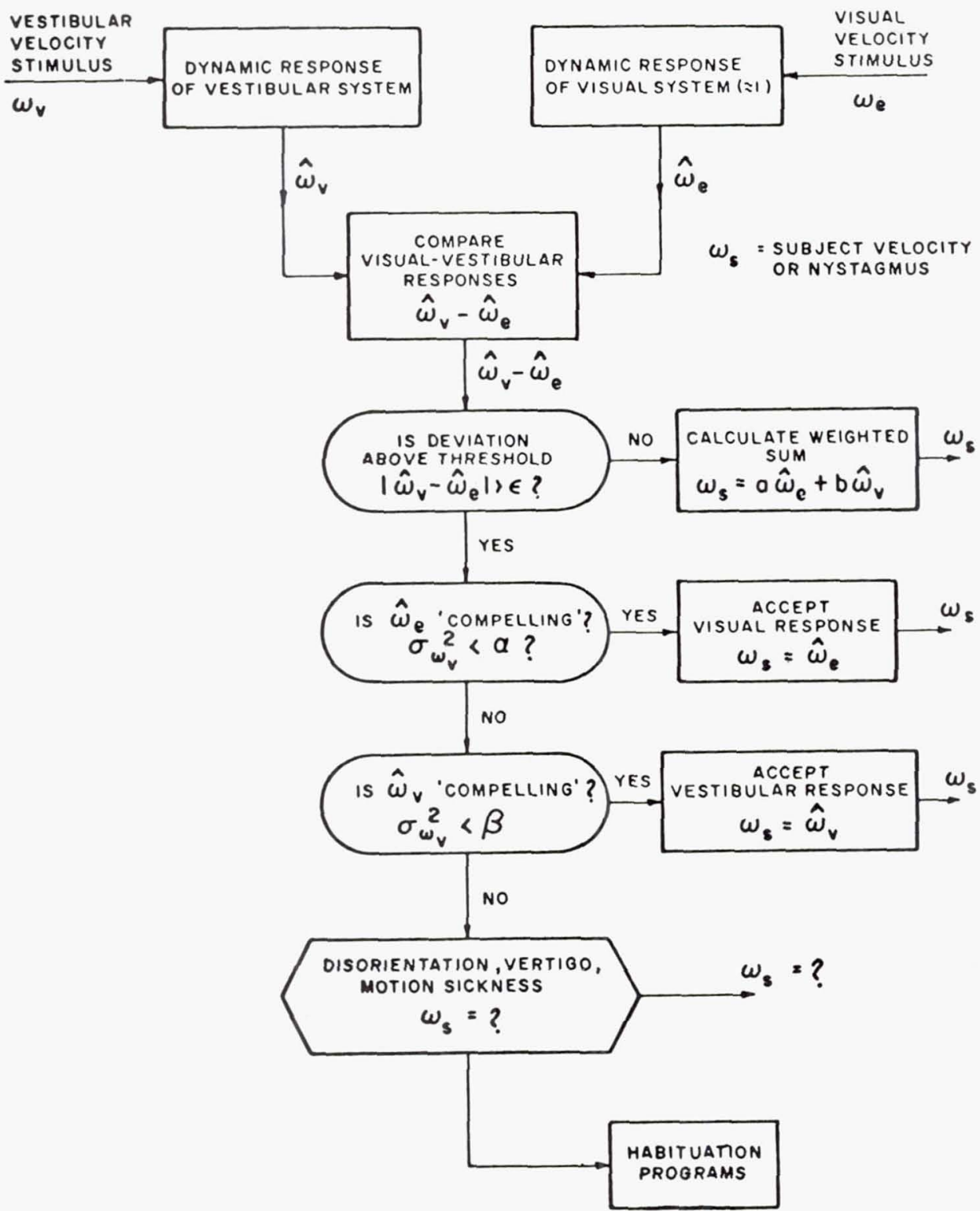

FIG. 25. Flow-chart representation of visual-vestibular interaction. [From Young (174).]

inverted situation), then disorientation, vertigo, and motion sickness might be expected to result.

Bischoff and Scheerer (10), in attempting to model visual-vestibular interactions for relative influences of visual fields in different head orientations, proposed a model that incorporated both the notion of linear weighting of visual and vestibular cues and also the idea that visual cues would be given greater weight in situations in which the otolith cues were associated with a high uncertainty. [It had already been demonstrated by Udo de Haes and Schöne (158) that the strength of visual orientation cues increased as head tilt varied from the erect position, and the strength of these cues generally followed the variability of the orientation perception based on otolith cues alone.] The systems analytic model of optical vestibular in- teraction in determining the vertical developed by Bischoff and Scheerer (10) and discussed by Bischoff (9) matches the perceived vertical data rather well. A somewhat modified version of this model is given in Figure 26. In this model, the compensatory signal C, which corrects the tilted retinal image of the target angle for head tilt, is generated on the basis of both feed-forward compensation from otolith cues regarding head tilt and feedback autocompensation based on the content of the image itself. The angle of the retinal image $\mathrm{R}$ would be equal to the target angle $\mathrm{T}$ except for the influence of head tilt $\mathrm{H}$, which is only partially compensated physically by ocular countertorsion E. The otolith system is shown to generate ocular torsion in a compensatory manner, and the sketch of E versus $\mathrm{H}$ is meant to imply that ocular torsion peaks at about 


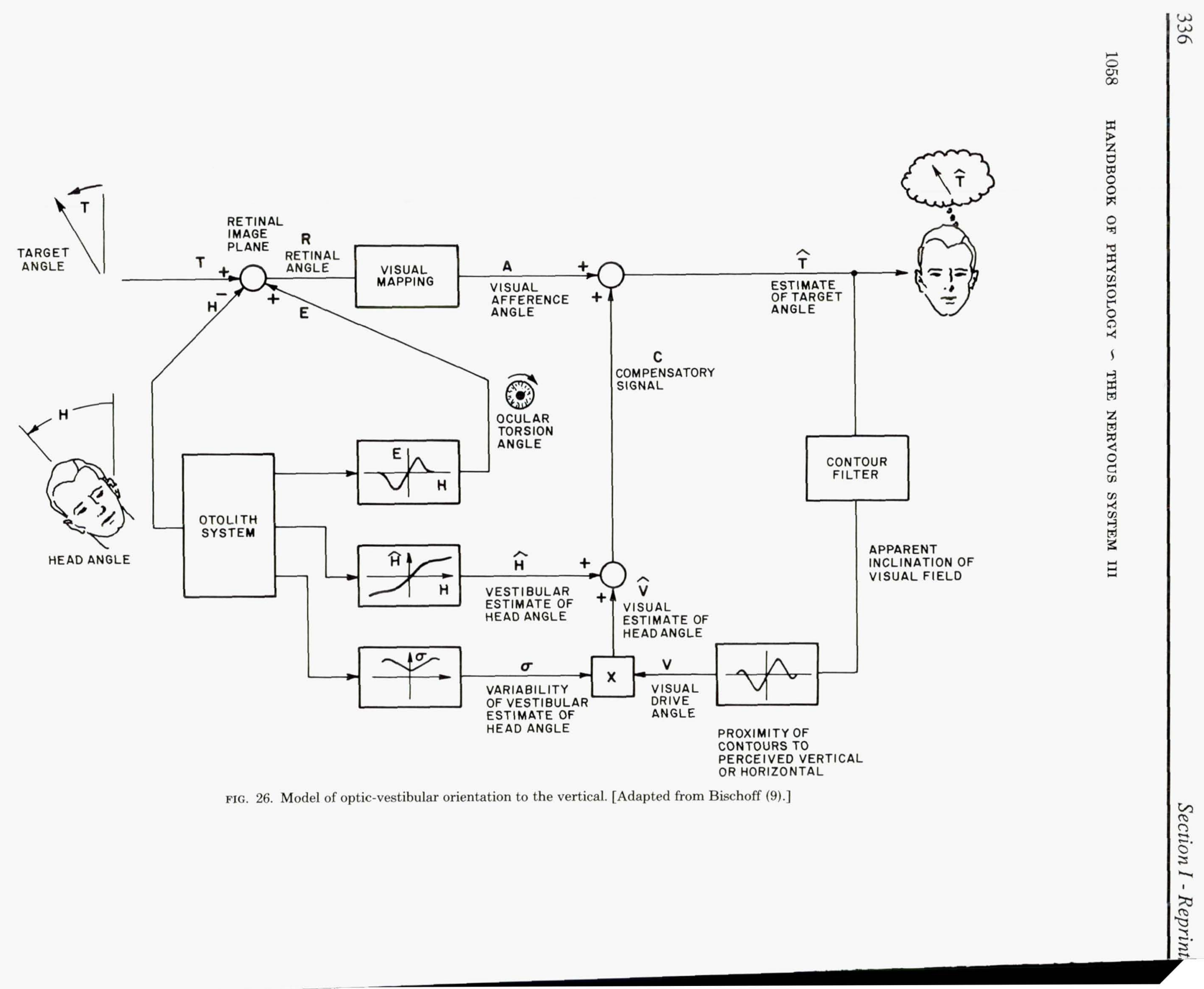


$60^{\circ}$ rather than at $90^{\circ}$, as would be predicted on the basis of lateral shear force on the utricular macula alone. It should be noted that no provision is made for ocular torsion to be influenced by the visual scene or the perceived head tilt angle, although these effects probably do occur.

The retinal angle $R$ is mapped to a cortical representation based on the visual afference $\mathrm{A}$, which must be corrected for the effects of $\mathrm{H}$ and $\mathrm{E}$ by a compensatory signal $\mathrm{C}$; the $\mathrm{C}$ is presumed to be the result of a linear weighting of the vestibular drive $\hat{\mathrm{H}}$ and the visual drive $\hat{\mathrm{V}}$. The vestibular portion of the compensatory signal is developed on the basis of otolith cues. The curve of $\hat{\mathrm{H}}$ versus $\mathrm{H}$ is meant to indicate the slight overestimation of tilt at small angles and the underestimation of tilt at larger angles of head tilt or the Müller and Aubert effects. The feedback autocompensation portion of this model assumes that the final estimate of target angle, which represents the perception of the target orientation in space, is passed through a contour filter to yield a signal representing the apparent inclination of the visual field with respect to the perceived vertical or horizontal. When the dominant axes lie elose to the vertical or horizontal, they are known to influence static orientation perception by pulling the perceived vertical toward the seen axis, and this is shown in the block "proximity of contours to perceived vertical or horizontal." The resulting visual drive angle $\mathrm{V}$ is multipled by $\sigma$, which represents the variability of the vestibular estimate of head angle. For the head erect, in which the vestibular signal has low variance and in which accurate estimates are made, the visual angle is consequently given little weight, whereas for the head tilted far from the vertical, $\sigma$ is larger and the visual weighting is greater, resulting in a larger signal $\hat{\mathrm{V}}$. Finally $\hat{\mathrm{V}}$, the visual estimate of head angle, is added linearly to $\mathrm{H}$ to produce $\mathrm{C}$.

Although this model has not apparently been tested for a wide variety of hypergravity and subgravity tests, nor is it meant to represent anatomical locations of the functions, it serves as a useful quantitative statement of the autocompensatory feedback notion combined with the reafference principle for compensating head angle.

\section{Vection and Dynamic Visual-Vestibular Interaction}

When a subject is moved actively or passively in conjunction with exposure to moving visual scenes, the resulting visual-vestibular interaction provides a rich test of the capabilities of the human spatial orientation system for dealing with normal and abnormal multisensory integration. Visual cues normally confirm the orientation information conveyed by the vestibular, proprioceptive, tactile, and motor command copy information. Thus, for example, when a subject tilts his head toward his right shoulder, the image of the stationary visual field on his retina rotates as though the external visual field were moved counterclockwise, his semicircular canals signal a transitory clockwise angular velocity that is integrated to yield an estimate of change in head angle and incidentally some compensatory eye torsion. Otolith signals register a new head orientation with respect to the vertical, confirming the semicircular canal and visual cues. Neck-joint angle receptors and possibly neck muscle spindles confirm the new head position and support the expectation, based on the internal model concept, that the commanded motor program was indeed fully affected. This represents a clear case of confirming visual and vestibular signals, leading to an unambiguous perception of spatial orientation. Similar comments apply to simple left-right head movements.

A wide variety of interesting and nontrivial conflicting visual-vestibular interaction cases may also be considered, although they have little to do with normal function. The normal relationship between head movement and relative movement of the visual field may be disturbed in a number of ways. When a subject wears left-right reversing prisms, field rotating prisms, magnifying or minifying spectacles (including reading glasses), the normal relationship between vestibular signals and visual signals on head rotation is disturbed. Rotation of the head under increased gravitational forces produces otolith signals that differ from the visual and semicircular canal representations of head movement. Head rotation in zero gravity fails to produce the normally expected tonic change in otolith activity to confirm the head movement. Nodding the head about an axis normal to that of continuing rotation produces the cross-coupled angular acceleration phenomenon discussed in Cross-Coupled Angular Acceleration; Coriolis Illusion, p. 1051, and creates motion illusions that conflict with the observed visualfield change. Visually induced self-motion in a flight simulator, which is not confirmed or only partially confirmed by concomitant vestibular cues, is another example of conflicting visual-vestibular interaction. Because the visual and vestibular inputs to motion perception can be easily controlled and because the perceptual and motor output (ocular stabilization and posture control) are relatively easily measured, visualvestibular interaction has been extensively studied as a paradigm for investigation of the more general question of multisensory integration $(45,86)$. The discussion that follows summarizes only a few of the more striking and unambiguous observations about the interaction between vestibular stimulation and motion sensation based on moving visual scenes.

The extent of visually induced tilt, either in pitch or roll, is limited by graviceptive cues that may not confirm this tilt. Thus the paradoxical sensation of rotation about an earth horizontal axis with only a limited illusion of induced tilt is attributable to the otolith signals. These, as well as other graviceptive systems, are presumably issuing conflicting commands indicating that head orientation is not changing rela- 
tive to the vertical. The central nervous system possibly compromises its orientation computation between a visual signal of ever-increasing tilt and a graviceptive signal indicating no tilt, and it arrives at some intermediate position. This phenomenon is analogous to the case described above for static field influence on the perceived direction of the vertical. When the visual field rotation is about a vertical axis, with the subject lying supine, the perception of rotation continues unabated with no limitation on the visually induced roll angle. Here, clearly, the otolith signals provide no information that confirms or denies the visually induced tilt. An even more powerful demonstration is the effectiveness of a rotating wide visual field on the onset and strength of continuous vection in weightless conditions, where no relevant otolith signals are present to confirm or deny the visual input (177). Intermediate head orientations, corresponding to positions in which estimates of the vertical based on graviceptive cues have high variance, showed stronger visually induced tilt (179), just as they showed stronger static visual scene influences $(10,157,158)$. All of these findings strengthen the view that visually induced tilt is limited by the lack of confirming otolith signals and that, when no such confirmation is to be expected (as in the case of zero gravity), the extent of the visual effect is not limited.

Similar limitations of the lack of confirming vestibular cues on visually induced motion are seen in yaw, where rotating visual fields induce circularvection. The delay in onset of circularvection and its gradual buildup is presumably the result of resolution of a conflict between visual motion signals and the absence of confirming semicircular canal cues. Visual-field acceleration at low levels, commensurate with semicircular canal signals close to threshold, do not show measurable latency, and even rapid visual scene acceleration can lead to immediate sensations of selfmotion if a small true vestibular cue is generated by slight body motion in the confirming direction. Conversely, the presence of an established circularvection can mask the detection of small body motions in the opposite direction and bias the perception of angular acceleration based on suprathreshold vestibular stimuli $(176,180)$. Finally, the relative domains of influence of visual and vestibular cues are separable and correspond to the frequency ranges in which each is a reliable transducer of self-motion. Continuous perception of velocity is supported by the low-frequency relative motion of the visual field, whereas vestibular cues, especially those from the semicircular canals, are adapted out and contribute relatively little low-frequency information. At high frequencies, on the other hand, reliance is much more upon vestibular than visual cues, especially when the two are in conflict. The rough division between high and low frequencies for this case is generally thought to lie in the vicinity of $0.1 \mathrm{~Hz}$.

A number of theoretical models for visual-vestibular interaction or for multisensory integration have been proposed (86). One of these compensation schemes, tested for static tilt of body and scene, was described in Static Visual-Vestibular Interaction, p. 1055. A more general notion, which has been discussed in a nonmathematical manner by many authors (e.g., refs. $76,144,174)$ is based on resolution of the conflict between visual and vestibular cues that results in changing the relative weighting applied to each of these sensory modalities. The weighting for each channel depends on the dynamic characteristics of all sensory channels and the amount of intersensory conflict. A mathematical treatment of the sensory conflict theory for spatial orientation that has been tested extensively for rotation about the vertical axis is presented in Figure 27 (180). In this model the gain, K, which controls the relative weighting of the visual system compared to the vestibular output, is adjusted according to a measure of cue conflict $\left(\omega_{\text {err }}\right)$. When the conflict between visual and vestibular signals is high, the relative weighting given to the vestibular input increases as $\mathrm{K}$ is reduced. In calculating the cue conflict, the difference is taken between the current vestibular signals, processed by the semicircular canals and indicating the vestibular system's estimate of angular velocity $\omega_{\text {ves }}$ and an appropriately filtered visual signal $\omega_{\text {vis. }}$ This visual signal is arrived at by passing the visual-field angular velocity through a first-order filter, which represents an internal model of the vestibular dynamics, to yield $\omega_{\text {vis. }}$ This signal corresponds to an internal representation of what the semicircular canal signals would be if the actual visual-field velocity were representative of head motion in a stationary field. The effect of this cue conflict adjustment of weighting function, which makes the model nonlinear, is to shift the weighting of sensory signals away from vestibular when the vestibular signals would either be highly variable or unlikely to present meaningful information about the relatively slow changes in true body velocity. On the other hand, where sudden changes in visual-field velocity are not borne out by actual vestibular signals on the basis of visual cues, then the visually induced motion is largely ignored in favor of reliance on the vestibular cues. Although this specific cue conflict model implementation has been developed primarily for modeling visual-vestibular interaction in rotation about a vertical axis, the concept of optimal mixing of multiple sensory cues appears to be generally valid and has been extended to other areas as well (15).

\section{SPATIAL ORIENTATION IN ALTERED ENVIRONMENTS}

Many of the illusions of spatial orientation associated with unusual or incompatible visual and vestibular stimuli are explainable on the basis of the conventional processing of sensory data. When these unusual sensory combinations persist for an extended period of time, from hours to days, the very meaning of the sensory signals in terms of spatial orientation is altered 


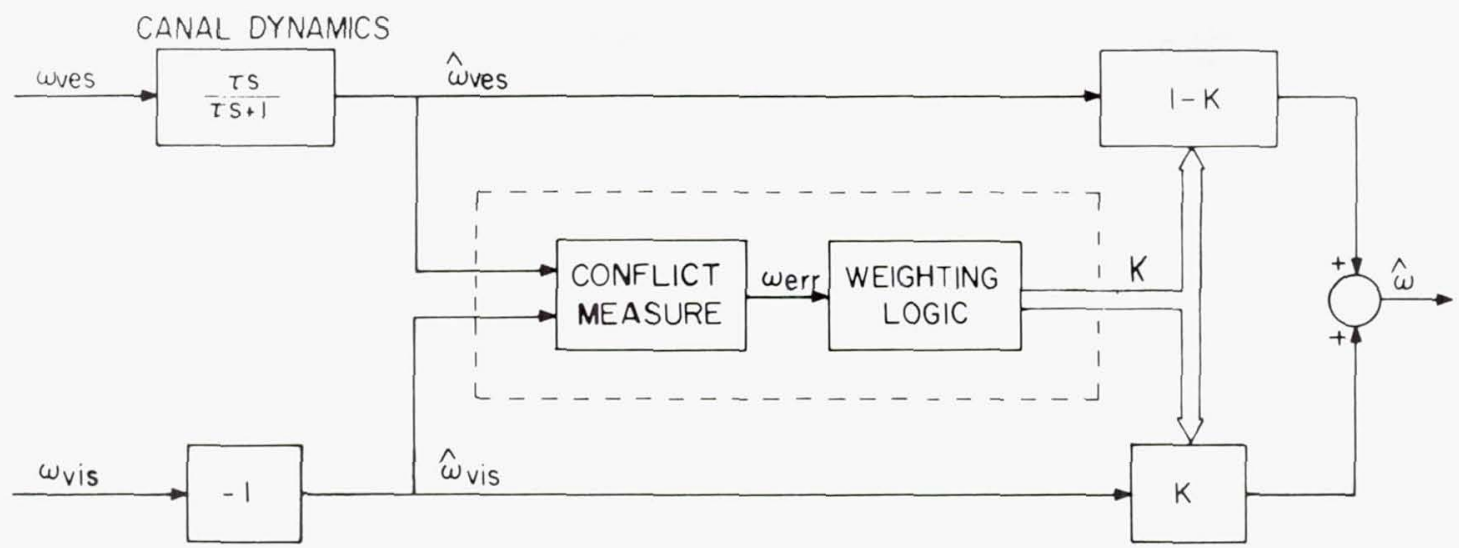

VISUAL DYNAMICS

A

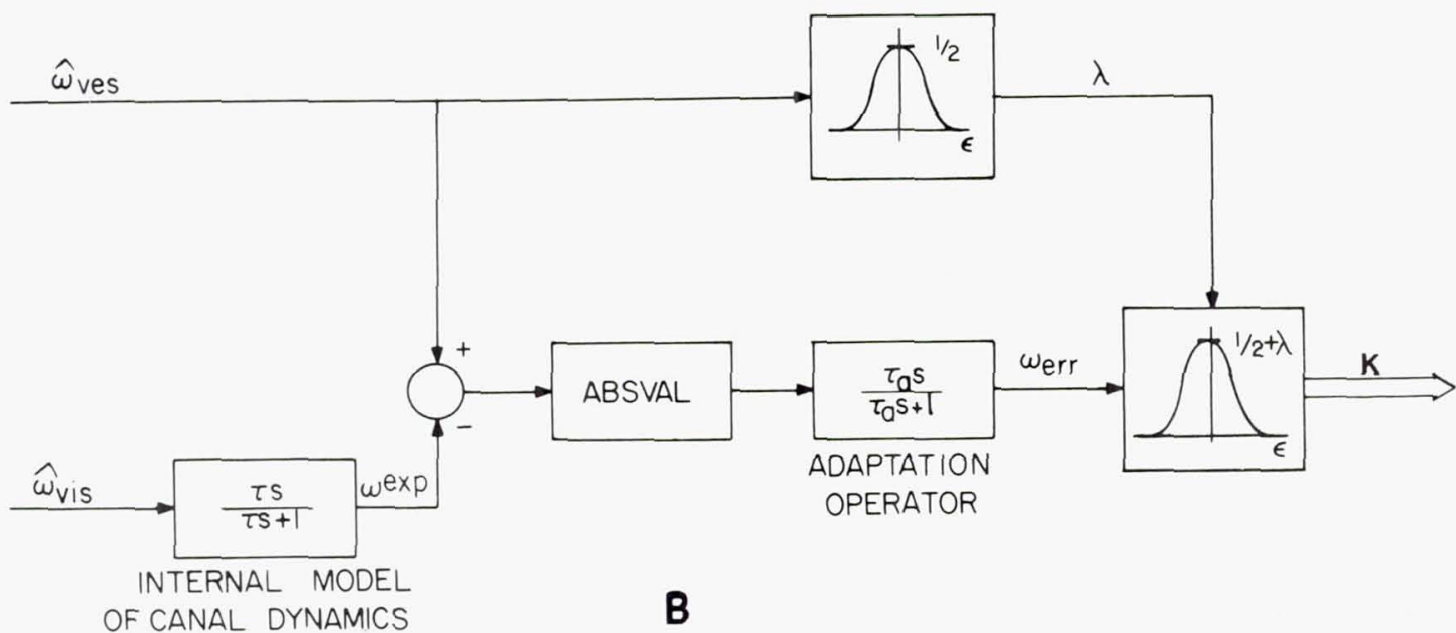

FIG. 27. Sensory conflict model for resolution of visual-vestibular interaction. $A$ : dual input conflict model; $B$ : conflict measure and weighting function. [From Zacharias and Young (180).]

and so is the manner in which perception takes place. The remarkable plasticity of animals to adapt to altered environments by altering sensorimotor functional pathways is particularly evident in the plasticity of the vestibuloocular reflex. Human spatial orientation shows a similar plasticity that allows one to adapt both orientation perception and posture control to various altered environments. An outstanding example of this plasticity is the adaptation of orientation to weightlessness after several days of space flight. The process of resolving the sensory rearrangement involves the formation of new internal models. This process is closely related to the topic of motion sickness, which occurs principally in unusual environments and in unexpected combinations of sensory stimuli. It is believed that the process of adaptation to altered spatial environments is closely linked to the process of overcoming motion sickness.

\section{Motion Sickness}

Humans and most animals can be made motion sick by exposure to a wide variety of motion and visual stimuli, provided the vestibular system is intact. Generally speaking, motion sickness occurs only under stimulus conditions associated with man-made vehicles and does not occur during motions typical of the normal range of natural head movements. A large number of fanciful theories for motion sickness and the remedies suggested by these theories are discussed in the excellent book on this subject by Reason and Brand (135). No satisfactory general theory for the survival value of motion sickness symptoms exists (154). It is by no means clear why motion sickness should in any way be tied to an evolutionary process, since it is a disorder resulting only from the use of technology. The neural pathways involved in motion sickness genesis are only slowly being worked out (66, 93, 120).

The most generally accepted current theory of motion sickness genesis is the conflict hypothesis. According to this theory, motion sickness is the result of conflict between spatial orientation signals coming from two or more different sources $(76,125,135,144)$. 
These conflicting signals may be within a sensory modality (semicircular canals versus otolith) or between sensory systems (visual versus vestibular). The conflict might also be between sensory signals and the anticipated motion based on motor output or between sensory information and the expected pattern of such signals based on previous experience or continuing motion. (As a simple example of the last case, consider the unusual vegetative response when an elevator begins to move in the unanticipated direction.) Airsickness, car sickness, and seasickness involve both intravestibular and visual-vestibular conflicts. The intravestibular conflicts arise from sustained linear or angular velocities at frequencies below the normal range of the semicircular canals or otoliths, particularly for airsickness. If the visual reference frame of the cabin or automobile is assumed inertially fixed, it is in conflict with the motion cues received from the otoliths and semicircular canals. Concentration on a visual task inside the cabin, such as reading, worsens the situation, whereas reference to an outside visual reference such as the roadway or horizon lessens the conflict and the symptoms if preventive action is taken early enough. (The reason that it is easy to read road signs but difficult to read a newspaper inside a bouncing bus is that the vestibular apparatus attempts to maintain ocular stability with respect to the outside visual field. This vestibular reaction must be suppressed and overridden by conflicting visuomotor reactions to permit concentration on the newspaper.)

Space motion sickness, which commonly occurs during the early days of weightless flight, is presumably attributable to the absence of sustained otolith cues to confirm head movements sensed by the visual system and the semicircular canals.

The process of adaptation to the altered environment, as in adaptation to seasickness, presumably involves some measure of reinterpretation of visual and vestibular signals and prediction of active or passive movement patterns. It is usefully expressed in terms of updating the internal model of the relationship between sensory signals and environmental stimuli. This reinterpretation can be very specific as to the frequency of motion or direction of turning and may provide no protection against sickness or disorientation from other stimuli. Land sickness is represented by unsteady gait, possible disorientation, and slight motion sickness symptoms when walking on steady ground after extensive time at sea. It presumably represents the carry-over of the (now inappropriate) internal model of predicted rolling of the ship and associated visual-vestibular interactions. Although a functioning vestibular system is necessary to produce motion sickness, stimulation of the vestibular appa- ratus is not required. Motion sickness symptoms may be elicited on the basis of large optokinetic stimuli, such as observed under laboratory conditions (43), in wide-screen movies and in wide-field-of-view fixedbase aircraft simulators $(3,119)$ or driving simulators. The principal conflict in these situations is between the vection generated by the moving visual field and the lack of confirming semicircular canal or otolith cues. The situation is worsened when the operator is an experienced pilot who presumably anticipates what the appropriate vestibular cues should be in response to a command and reacts to the conflict associated with their nonappearance.

The conflict theory for motion sickness has been extended and cast in control theory terms by Oman (125). Motion sickness symptoms are also generated by head movements under various unusual environments in which the normal pattern of intravestibular or visual-vestibular correlations are disturbed. Conflicting cues are created by wearing left-right reversing prisms or by performance of head movements during weightlessness or in a slowly rotating room. In each of these cases, the vestibular reactions--oculomotor, postural, and perception-do not conform with the observed visual patterns. The subsidence of motion sickness symptoms appears to parallel the development of new internal models and the ability to make appropriate directed movements. The spatial orientation system demonstrates its remarkable versatility in its plastic adaptation to altered environments or even to loss of major portions of the sensory system.

\section{SUMMARY}

Spatial orientation is firmly based on the underlying sensory mechanisms and their central integration. For some of the simplest situations, like rotation about a vertical axis in darkness, the dynamic response of the semicircular canals furnishes almost enough information to explain the sensations of turning and stopping. For more complex conditions involving multiple sensory systems and possible conflicts among their messages, a mechanistic response requires significant speculative assumptions. The models that exist for multisensory spatial orientation are still largely of the nonrational parameter variety. They are capable of predicting relationships among input motions and output perceptions of motion, but they involve computational functions that do not now and perhaps never will have their counterpart in central nervous system machinery. The challenge continues to be in the iterative process of testing models by experiment, correcting them where necessary, and testing them again.

\section{REF E R E N C S}

1. Aubert, H. Eine scheinbare bedeutende Drehung von Objekten bei Neigung des Kopfes nach rechts oder links. Virchows Arch. 20: 381-393, 1861.

2. BÁRÁNY, R. New methods of examination of the semicircular canals and their practical significance. Ann. Ophthalmol. 16: $755-861,1907$. 
3. Barrett, G. V., and G. L. Thornton. Relationship between perceptual style and simulator sickness. J. Appl. Psychol. 52: 304-308, 1968.

4. Benson, J. Modification of the response to angular accelerations. In: Handbook of Sensory Physiology. Vestibular System, edited by H. H. Kornhuber. New York: Springer-Verlag, 1974, vol. 6 , pt. 2 , p. 281-320.

4a.Benson, A. J. Perceptual illusions. In: Aviation Medicine, edited by G. Dhenin. London: Tri-Med Books, 1978.

5. Benson, A. J., and G. R. Barnes. Responses to Rotating Linear Acceleration Vector Considered in Relation to a Model of the Otolith Organs. Washington, DC: U.S. Aeronaut. and Space Admin., 1973, SP-314, p. 221-236.

6. BEnson, A. J., AND M. A. Bodin. Interaction of linear and angular acceleration on vestibular receptors in man. Aerosp. Med. 37: 144-154, 1966.

7. Benson, A. J., And F. E. Guedry. Comparison of tracking task performance and nystagmus during sinusoidal oscillations in yaw and pitch. Aerosp. Med. 42: 593-601, 1971.

8. Berthoz, A., L. R. Young, and F. Oliveras. Action of alcohol on vestibular compensation and habituation in the cat. Acta Oto-Laryngol. 84: 317-327, 1977.

9. Bischoff, N. Optic-vestibular orientation to the vertical. In: Handbook of Sensory Physiology. Vestibular System, edited by H. H. Kornhuber. New York: Springer-Verlag, 1974, vol. 6, pt. 2, p. $155-192$.

10. Bischoff, N., and E. Scheerer. Systemanalyse der optischvestibulären Interaktion bei der Wahrnehmung der Vertikalen. Psychol. Forsch. 34: 99-181, 1970.

11. Bitterman, M. E., and P. Worchel. The phenomenal vertical and horizontal in blind and sighted subjects. Am. J. Psychol. 66: 598-602, 1953.

12. Blanks, R. H. I., I. S. Curthoys, and C. H. Markham. Planar relationships of the semicircular canals in man. Acta Oto-Laryngol. 80: 185-196, 1975.

13. Bock, O., AND W. H. Zangemeister. A mathematical model of air and water caloric nystagmus. Biol. Cybern. 31: 91-95, 1978.

14. Borah, J., L. R. Young, and R. E. Curry. Sensory Mechanism Modelling. Dayton, $\mathrm{OH}$ : Wright-Patterson AFB, Adv. Syst. Div., 1977. (AFHRL-TR-77-70.)

15. Borah, J., L. R. Young, AND R. E. CuRRY. Optimal estimator model for human spatial orientation. IEEE Trans. Syst. Man Cybern. In press.

16. Boring, E. G. Sensation and Perception in the History of Experimental Psychology. New York: Appleton, 1942.

17. Brandt, Th., J. Dichgans, and E. Koenig. Differential effects of central versus peripheral vision on egocentric and exocentric motion perception. Exp. Brain Res. 16: 476-491, 1973.

18. Brandt, Th., H. C. Diener, and J. Dichgans. Motion sickness induced through angular oscillations of the body or the visual surround in normals and after labyrinthine lesions. Int. Congr. Aviat. Space Med., 23rd, Acapulco, 1975.

19. Brandt, Th., E. R. Wist, and J. Dichgans. Foreground and background in dynamic spatial orientation. Percept. Psychophys. 17: 497-503, 1975 .

20. Brown, J. L. Orientation to the vertical during water immersion. Aerosp. Med. 32: 209-217, 1961.

21. BüHLER, K. Die Erscheinungsweisen der Farben. Jena, Germany: Fischer, 1922.

22. Büttner, U., And V. Henn. Thalamic unit activity in the alert monkey during natural vestibular stimulation. Brain Res. 103: 127-132, 1976.

23. Büttner, U., V. Henn, and L. R. Young. Frequency response of the vestibulo-ocular reflex (VOR) in the monkey. Aviat. Space Environ. Med. 52: 73-77, 1981.

24. Büttner, U., V. Henn, And H. P. Oswald. Vestibular related neuronal activity in the thalamus of the alert monkey during sinusoidal rotation in the dark. Exp. Brain Res. 30: 435-444, 1977.

25. Bürtner, U., W. Waespe, and T. S. Miles. Transfer characteristics of the vestibular system determined from nystagmus and neuronal activity in the alert monkey. In: Kybernetic '77, edited by E. Butenand and G. Hauske. Munich: Oldenbourg, 1978 , p. 126-136.

26. Byford, G. H. Eye movements and the optogyral illusion. Aerosp. Med. 34: 119-123, 1963.

27. CAPPEL, K. Determination of Physical Constants of Semicir cular Canals From Measurement of Single Neural Unit Activity Under Constant Angular Acceleration. Washington, DC: U.S. Aeronaut. and Space Admin., 1966, SP-115, p. 229236.

28. Cawthorne, T., M. Dix, C. Hallpike, and J. Hood. The investigation of vestibular function. Br. Med. Bull. 12: 131-142, 1956.

29. Chambers, M. R., K. H. Andres, M. Deuring, and A. Iggo. The structure and function of the slowly adapting type II mechanoreceptor in hairy skin. Q. J. Exp. Physiol. 57: 417-455, 1972.

30. Clark, B., and A. Graybiel. Perception of postural vertical following prolonged bodily tilt in normals and subjects with labyrinthine defects. Acta Oto-Laryngol. 58: 143-148, 1964.

31. Clark, B., and J. D. Stewart. Effects of angular rotation on man. Thresholds for the perception of rotation and the oculogyral illusion. Aerosp. Med. 40: 952-956, 1969.

32. Coats, A. C., AND S. Y. Smith. Body position and the intensity of caloric nystagmus. Acta Oto-Laryngol. 63: 515-532, 1967.

33. Cohen, B., T. Uemera, and S. TAKemori. Effects of labyrinthectomy on optokinetic nystagmus (OKN) and optokinetic afternystagmus (OKAN). Equil. Res. 3: 88-93, 1973.

34. Collins, W. E., AND F. E. GuedRY. Duration of angular acceleration and ocular nystagmus from cats and man. I. Responses from the lateral and vertical canals to two stimulus durations. Acta Oto-Laryngol. 64: 373-387, 1967.

35. Correia, M. J., AND F. E. Guedry, JR. Modification of Vestibular Responses as a Function of Rate of Rotation About an Earth-Horizontal Axis. Pensacola, FL: Naval Aerosp. Med. Inst., 1966. (NAMI Rep. 957.)

36. Correia, M. J., W. C. Hixson, and J. I. Niven. Otolith Shear and the Visual Perception of Force Directions: discrepancies and a Proposed Resolution. Pensacola, FL: Naval Aerosp. Med. Inst., 1965. (NAMI Rep. 951.)

37. Correia, M. J., W. C. Hixson, and J. I. Niven. On predictive equations for subjective judgments of vertical and horizontal in a force field. Acta Oto-Laryngol. Suppl. 230: 1-20, 1968.

38. Correia, M. J., J. P. Landolt, M.-D. Ni, A. R. Eden, and J. L. RAE. A species comparison of linear and nonlinear transfer characteristics of primary afferents innervating the semicircular canal. In: Vestibular Function and Morphology, edited by T. Gualtierotti. New York: Springer-Verlag, 1981, chapt. 16, p. 280-316.

39. Correia, M. J., J. B. Nelson, and F. E. Guedry, Jr. The antisomatogyral illusion. Aviat. Space Environ. Med. 48: 859$862,1977$.

41. Daunton, N. G., D. D. Thomsen, and C. A. Christensen Visual vestibular interaction in vertically sensitive otolith-dependent units. Soc. Neurosci. Abstr. 5: 690, 1979.

42. DeVries, H. L. The mechanics of the labyrinth otoliths. Acta Oto-Laryngol. 38: 263-273, 1950.

43. Dichgans, J., AND Th. Brandt. Optokinetic motion sickness and pseudo-Coriolis effects induced by moving visual stimuli Acta Oto-Laryngol. 76: 339-348, 1973.

44. Dichgans, J., AND Th. BRAndt. The psychophysics of visually induced perception of self-motion and tilt. In: The Neurosci ences: Third Study Program, edited by F. O. Schmitt and F. G. Worden. Cambridge, MA: MIT Press, 1974, p. 123-129.

45. Dichgans, J., AND Th. Brandt. Visual vestibular interaction effects on self-motion perception and postural control. In Handbook of Sensory Physiology. Perception, edited by $\mathrm{R}$ Held, H. Liebowitz, and H. L. Teuber. New York: SpringerVerlag, 1978, vol. 8, p. 775-804.

46. Dichgans, J., R. Held, L. R. Young, and T. Brandt. Moving visual scenes influence the apparent direction of gravity. Science 178: 1217-1219, 1972

47. Dohlman, G. Towards a method for quantitative measure- 
ment of the functional capacity of the vestibular apparatus. Acta Oto-Laryngol. 23: 50-62, 1935.

48. Ewald, J. R. Physiologische Untersuchungen über das Endorgan des Nervus Octavus. Wiesbaden, Germany: Bergmann, 1892.

48a.Fechner, G. T. Elements der Psychophysik. Leipzig: Breitkopf \& Härtel, 1860, 2 vol. Elements of Psychophysics, transl. by H. E. Adler. New York: Holt, Reinhart, 1966.

49. Fernández, C., and J. M. Goldberg. Physiology of the peripheral neurons innervating the semicircular canals of the squirrel monkey. The response to sinusoidal stimulation and dynamics of the peripheral vestibular system. J. Neurophysiol. 34: 661-675, 1971.

50. Fernández, C., and J. M. Goldberg. Physiology of peripheral neurons innervating otolith organs of the squirrel monkey. Parts I, II, and III. J. Neurophysiol. 39: 970-1008, 1976.

51. Fischer, M. H. Messender Untersuchungen über die Gegenrollung der Augen und die Lokalisation der scheinbaren Vertikalen bei seitlicher Neigung (des Kopfes, des Stammes, und Gesamtkörpers). I. Neigungen bis zu $40^{\circ}$. Von Graefes Arch. Ophthalmol. 118: 633-680, 1927.

52. Fischer, M. H. Messender Untersuchungen über die Gegenrollung der Augen und die Localisation der scheinbaren Vertikalen bei seitlicher Neigung des Gesamtkörpers bis zu $360^{\circ}$. II. Mitteilung, Untersuchungen an Normalen. Von Graefes Arch. Ophthalmol. 123: 476-508, 1930.

53. Fischer, M. H. Messender Untersuchungen über die Gegenrollung der Augen und die Lokalisation der scheinbaren Vertikalen bei seitlicher Neigung des Körpers, Kopfes und Stammes. III. Mitteilung, Untersuchungen an einem einseitig Labyrinthlosen. Von Graefes Arch. Ophthalmol. 123: 509-531, 1930.

54. Fischer, M. H., ANd A. Kornmüller. Optokinetisch ausgelöste Bewegungswahrnehmungen und optokinetischer Nystagmus. J. Psychol. Neurol. 41: 273-308, 1930.

55. Flourens, J. M. P. Experiences sur les canaux semicirculaire de l'orielle dans les mammifères. Acad. R. Sci. Paris 9: 467477,1830 .

56. Flourens, J. M. P. Recherches expérimentales sur les proprietes du système nerveux dans l'animaux vertebrés (2nd ed.). Paris: Baillière, 1842

57. Fluur, E., and A. Mellström. Saccular stimulation and oculomotor reactions. Laryngoscope 80: 1713-1721, 1970.

58. Fraenkel, G. S., and D. L. Gunn. The Orientation of Animals. New York: Dover, 1940.

59. Fuchs, A. F., AND H. H. Kornhuber. Extraocular muscle afferents to the cerebellum of the cat. J. Physiol. London 200: 713-722, 1969.

60. Gibson, J. J., AND M. Radner. Adaptation, after-effects and contrast in the perception of tilted lines. J. Exp. Psychol. 20: 452-467, 553-569, 1937.

61. Gilllingham, K. A Primer of Vestibular Function, Spatial Disorientation and Motion Sickness. San Antonio, TX: Brooks AFB, US Air Force Sch. of Aviat. Med., 1966. (SAM Aeromed. Rev. 4-66.)

62. Gillingham, K., AND R. W. Krutz. Effects of the Abnormal Acceleratory Environment of Flight. San Antonio, TX: Brooks AFB, US Air Force Sch. of Aviat. Med., 1977. (SAM Aeromed. Rev. 10-74.)

63. Gonshor, A., And G. Melvill-Jones. Changes of human vestibuloocular responses induced by vision reversal during head rotation. J. Physiol. London 234: 102P-103P, 1973.

64. Gonshor, A., and G. Melvill-Jones. Extreme vestibuloocular response induced by prolonged optical reversal of vision. J. Physiol. London 256: 381-414, 1976.

65. Goodwin, G. M., D. I. McClaskey, and P. B. C. Matthews. The contributions of muscle afferents to kinesthesia shown by vibration induced illusions of movement and by the effects of paralyzing joint afferents. Brain 95: 705-708, 1972.

66. GRAYBIEL, A. Measurement of otolith function. In: Handbook of Sensory Physiology. Vestibular System, edited by H. H. Kornhuber. New York: Springer-Verlag, 1974, vol. 6, pt. 2, p.

\section{3-266.}

67. Graybiel, A., and R. H. Brown. The delay in visual reorientation following a change in direction of the resultant force on a human centrifuge. J. Gen. Psychol. 45: 143-150, 1951.

68. Graybiel, A., W. A. Kerr, and S. H. Bartley. Stimulus threshold of the semicircular canal as a function of angular acceleration. Am. J. Psychol. 61: 21-36, 1948.

69. Graybiel, A., E. F. Miller II, J. Billingham, R. Waite, C. A. Berry, and L. F. Deitlein. Vestibular experiments in Gemini flights V and VII. Aerosp. Med. 38: 360-370, 1967.

70. Graybiel, A., E. F. Miller, II, AND J. L. Homick. Experiment M-131. Human Vestibular Function. NASA TMX-58154. Washington, DC: U.S. Aeronaut. and Space Admin., 1974, p. 169-220. (Proc. Skylab Life Sci. Symp.)

71. Graybiel, A., E. F. Miller II, B. D. Newsom, and R. S. KENNEDY. The effect of water immersion on perception of the oculogravic illusion in normal and labyrinthine defective subjects. Acta Oto-Laryngol. 65: 599-610, 1968.

72. Groen, J. J. Adaptation. Pract. Oto-Rhino-Laryngol. 19: 524$530,1957$.

73. Groen, J. J. Vestibular stimulation and its effects from the point of view of theoretical physics. Confin. Neurol. 21: 380389, 1969.

74. Groen, J. J., And L. B. W. Jongkees. The threshold of angular acceleration perception. J. Physiol. London 107: 1-7, 1948.

75. Guedry, F. E. Orientation of the rotation axis relative to gravity: Its influence on nystagmus and the sensation of rotation. Acta Oto-Laryngol. 60: 30-48, 1965

76. Guedry, F. E. Psychophysics of vestibular stimulation. In: Handbook of Sensory Physiology. Vestibular System, edited by H. H. Kornhuber. New York: Springer-Verlag, 1974, vol. 6, pt. 2, p. 3-154.

77. Guedry, F. E., And A. J. Benson. Coriolis cross-coupling effects: disorienting and nauseogenic or not? Aviat. Space Environ. Med. 49: 29-35, 1978.

78. Guedry, F. E., and S. J. Ceran. Derivation of Subjective Velocity from Angular Displacement Estimates Made During Prolonged Angular Acceleration: Adaptation Effects. Fort Knox, KY: US Aviat. Med. Res. Lab., 1958. (USAMRL Rep. 376.)

79. Guedry, F. E., AND W. E. Collins. Duration of angular acceleration and ocular nystagmus in cat and man. Acta OtoLaryngol. 65: 257-269, 1968.

79a.GUEDRY, F. E., AND L. S. LAUver. Vestibular reactions during prolonged constant angular acceleration. J. Appl. Physiol. 16: $215-220,1961$.

80. Guedry, F. E., C. E. Mortenson, J. B. Nelson, and M. J. CoRreia. A comparison of nystagmus and turning sensations generated by active and passive turning. In: Vestibular Mechanisms in Health and Disease, edited by J. D. Hood. New York: Academic, 1978, p. 317-325.

81. Guedry, F. E., G. G. Owens, and J. W. Norman. Assessment of Semicircular Canal Function. I. Measurement of Subjective Effects Produced by Triangular Waveforms. Pensacola, FL: US Naval Aerosp. Med. Inst., 1969. (NAMI-1073.)

82. Guedry, F. E., C. W. Stockwell, and R. D. Gilson, Comparison of subjective responses to semicircular canal stimulation produced by rotation about different axes. Acta OtoLaryngol. 72: 101-106, 1971.

83. Guedry, F. E., C. W. Stockwell, J. W. Norman, and G. G. OWENS. Use of triangular waveforms of angular velocity for the study of vestibular function. Acta Oto-Laryngol. 71: 439448,1971

84. Held, R., J. Dichgans, and J. Bauer. Characteristics of moving visual scenes influencing spatial perception. Vision Res. 15: 357-365, 1975.

85. Helmholtz, H. von. Handbuch der physiologischen Optik. Hamburg: Voss, 1896.

86. Henn, V., B. Cohen, and L. R. Young. Visual vestibular interaction in motion perception and the generation of nystagmus. Neurosci. Res. Program Bull. 18: 459-651, 1980. 
87. Henn, V., And L. R. Young. Ernst Mach on the vestibular system 100 years ago. ORL-J. Oto-Rhino-Laryngol. Its Borderl. 37: 138-148, 1975.

88. Hillman, D. E., and J. W. McLaren. Displacement configuration of the semicircular canal cupulae. Neuroscience 4: 1989$2000,1979$.

89. Hixson, W., J. Niven, And M. Correia. Kinematic Nomen clature for Physiological Accelerations with Special Refer ence to Vestibular Applications. Pensacola, FL: US Naval Aeromed. Inst., 1966. (Monograph 14.)

90. Howard, I. P., and W. B. Templeton. Human Spatial Orientation. New York: Wiley, 1966.

91. Huang, J. K., And L. R. Young. Sensation of rotation about a vertical axis with a fixed visual field in different illuminations and in the dark. Exp. Brain Res. 41: 172-183, 1981.

92. IGGo, A., AND A. R. Muir. The structure and function of a slowly adapting touch corpuscle in hairy skin. J. Physiol. London 300: 762-769, 1969.

93. Johnson, W. H. Motion sickness. Part I. Aetiology and autonomic effects. In: Handbook of Sensory Physiology. Vestibular System, edited by H. H. Kornhuber. New York: SpringerVerlag, 1974, vol. 6, pt. 2, p. 389-404.

94. Jongkees, L. B. W., AND J. J. GRoEn. The nature of the vestibular stimulus. J. Laryngol. 61: 529-541, 1946.

95. KaRDOS, L. Ding Farbenwahrnehmung und Duplizatätstheorie. $Z$. Psychol, 108: 240, 1928.

96. Kardos, L. Die "konstanz" phänomenaler Dingmoment. In Beiträge zur Problemgeschichte der Psychologie, edited by E. Brunswick. Jena, Germany: Fischer, 1929.

96a.Kellogg, R. S. The Role of Vestibular Organs in the Exploration of Space. Washington, DC: U.S. Aeronaut. and Space Admin., 1965, SP-77, p. 195-202.

97. KeLlogG, R. S., AND A. Graybiel. Lack of response to thermal stimulation of the semicircular canals in the weightless phase of parabolic flight. Aerosp. Med. 38: 487-490, 1967.

98. Kleint, H. Versuche über die Wahrnehmung. Z. Physiol. 138 $1-34,1937$

99. KuIx, F. Elementaranalysen zur Psychophysik der Raum wahrnehmung. Berlin: Dtsch. Verlag Wissensch., 1962.

100. Kron, G. J., and J. M. KleinwaKs. Development of the advanced g-cuing system. Presented at the AIAA Flight Simulation Conf. Arlington, TX, 1978.

101. LaCkNer, J., AND M. S. Levine. Changes in apparent body orientation and sensory localization induced by vibration of postural muscles: vibratory myesthetic illusions. Aviat. Space Environ. Med. 50: 346-54, 1979.

102. Lechner-Steinleitner, S., H. Schöne, and N. J. Wade. Perception of the visual vertical: utricular and somatosensory contributions. Psychol. Rev, 40: 407-414, 1979.

103. Ledoux, A. Activité électrique des nerfs des canaux semicirculaire du saccule et de l'utricle chez la grenouille. Acta OtoRhino-Laryngol. Belg. 3: 335-349, 1949

104. Loeb, J. Forced Movements, Tropism and Animal Conduct Philadelphia and London, 1918.

105. Lowenstein, O. E. Physiology of vestibular receptors. In: Progress in Brain Research, Basic Aspects of Central Vestibular Mechanisms, edited by A. Brodal and O. Pompeiano. Amsterdam: Elsevier, 1972, vol. 37, p. 19-30.

106. MACH, E. Grundlinien der Lehre von den Bewegungsempfinden. Leipzig: Englemann, 1875.

107. Makarov, P. O., and D. S. Motoyan. Topaxia: the significance of the spatial factor in the excitability of the cutaneous sensory system in man. Biofizika 13: 662-669, 1968.

108. Malcolm, R., AND G. Melvill-Jones. A quantitative study of vestibular adaptation in humans. Acta Oto-Laryngol. 70: $126-135,1970$.

109. Malcolm, R., and G. Melvill-Jones. Erroneous perception of vertical motion by humans seated in the upright position. Acta Oto-Laryngol. 77: 274-283, 1974.

110. Mann, C. W., and H. J. Dauterive. The perception of the vertical. I. The modification of non-labyrinthine cues. J. Exp. Psychol. 39: 700-707, 1949.
111. MaYNe, R. A systems concept of the vestibular organs. In: Handbook of Sensory Physiology, Vestibular System, edited by H. H. Kornhuber. New York: Springer-Verlag, 1974, vol. 6 , pt. 2, p. $493-560$.

112. Meiry, J. L. The Vestibular System and Human Spatial Orientation. Cambridge: Massachusetts Inst. of Technol., 1965. Sc.D. thesis.

113. Melvill-Jones, G. Origin, significance and amelioration of Coriolis illusions from the semicircular canals: a nonmathematical appraisal. Aerosp. Med. 41: 483-490, 1970.

114. Melvill--Jones, G., W. Barry, and N. Kowalsky. Dynamics of the semicircular canals compared in yaw, pitch and roll. Aerosp. Med. 35: 984-989, 1964.

115. Melvill-Jones, G., And J. H. Milsum. Characteristics of neural transmission from the semicircular canal to the vestibular nuclei of cats. J. Physiol. London 209: 295-319, 1969.

116. Melvill-Jones, G., AND K. E. Spelds. A theoretical and comparative study of the functional dependence of the semicircular canal upon its physical dimensions. Proc. R. Soc. London Ser. B 157: 403-419, 1963.

117. Melvill-Jones, G., AND L. R. Young. Subjective detection of vertical acceleration: A velocity dependent response? Acta Oto-Laryngol. 85: 45-53, 1978.

118. Miller, E. F., II, And A. Graybiel. Magnitude of gravitoinertial force, an independent variable in egocentric visual localization of the horizontal. J. Exp. Psychol. 71: 452-460, 1966.

119. Miller, J. W., AND J. E. Goodson. Motion sickness in a helicopter simulator. Aerosp. Med. 31: 204-211, 1960.

120. Money, K. E. Motion sickness. Physiol. Rev. 50: 1-39, 1970.

121. Money, K. E., AND W. S. Myles. Heavy water nystagmus and effects of alcohol. Nature London 247: 404-405, 1974.

122. Mountcastle, V. B. The problem of sensing and the neural coding of sensory events. In: The Neurosciences. New York: Rockefeller Univ. Press, 1967

123. Müller, G. E. Über das Aubertsche Phänomen. Z. Sinnesphysiol. 49: 109-244, 1916.

124. Oman, C. M. Dynamic Response of the Semicircular Canals and Lateral Line Organs. Cambridge: Massachusetts Inst. of Technol., 1972. Ph.D. thesis.

125. OMAN, C. M. A heuristic mathematical model for the dynamics of sensory conflict and motion sickness. Acta Oto-Laryngol. Suppl. 392, 1982.

126. OMAN, C. M., O. Bock, AND J. K. HuANG. Visually induced self-motion sensation adapts rapidly to left-right vision reversal. Science 209: 706-708, 1980.

127. Oman, C. M., L. S. Frishropf, and M. Goldstein. Cupula motion in the semicircular canal of the skate, Raja erinacea: an experimental investigation. Acta Oto-Laryngol. 87: 528-538, 1979.

128. Oman, C. M., AND Young, L. R. Physiologic range of pressure difference and cupula deflections in the human semicircular canals. In: Progress in Brain Research. Basic Aspects of Central Vestibular Mechanisms, edited by A. Brodal and O. Pompeiano. Amsterdam: Elsevier, 1972, vol. 37, p. 539-549.

129. OMAN, C. M., AND L. R. Young. Physiological range of pressure difference and cupula deflections in the human semicircular canals: theoretical considerations. Acta Oto-Laryngol. 74: 324$331,1972$.

130. Oosterveld, W. J., and W. D. VAN Der LaArse. Effect of gravity on vestibular nystagmus. Aerosp. Med. 40: 382-385, 1969

131. Ormsby, C. C. Model of Human Dynamic Orientation. Cambridge: Massachusetts Inst. of Technol., 1974. Ph.D. thesis.

132. ORmSBy, C. C., AND L. R. Young. Perception of static orientation in a constant gravitoinertial environment. Aviat. Space Environ. Med. 47: 159-164, 1976.

133. Parker, D. E. The vestibular apparatus. Sci. Am. 243: 118$135,1980$.

134. Poulton, E. C. The new psychophysics: six models for magnitude estimation. Psychol. Bull. 69: 1-19, 1968.

135. Reason, J. T., and J. J. Brand. Motion Sickness. New York: 
Academic, 1975.

136. Roggeveen, L. J., ANd P. Nishoff. The normal and pathological threshold of the perception of angular accelerations for the optogyral illusion and the turning sensation. Acta OtoLaryngol. 46: 533-541, 1956.

137. Schneider, C. W., AND S. H. Bartley. A study of the effects of mechanically induced tension of the neck muscles on the perception of verticality. J. Psychol. 52: 245-248, 1962.

138. Sсноск, G. J. D. Perception of the horizontal and vertical in simulated subgravity conditions. US Armed Forces Med. J. 11: 786-793, 1960.

139. Schöne, H. Über den Einfluss der Schwerkraft auf die Augenrollung und auf die Wahrnehmung der Lage im Raum. Z. Vgl. Physiol. 46: 57-87, 1962.

140. SCHÖNE, H. On the role of gravity in human spatial orientation. Aerosp. Med. 35: 764-772, 1964.

141. Schöne, H. Orientierung in Raum. Stuttgart, West Germany: Wissenschaftliche Verlagsgesellschaft., 1980.

142. Schöne, H., AND H. Udo de Haes. Space orientation in humans with special reference to the interaction of vestibular, somaesthetic and visual inputs. Biokybernetik 3: 172-191, 1971.

143. Solley, C. M. Reduction of error with practice in perception of the postural vertical. J. Exp. Psychol. 52: 329-333, 1956.

144. Steele, J. E. Motion sickness and spatial perception-a theoretical study. In: Symposium on Motion Sickness with Special Reference to Weightlessness. Dayton, OH: Wright-Patterson AFB, 1963. (ASD Tech. Rep. 61-530.)

145. Steer, R. W. Progress in Vestibular Modelling. Part I. Responses of Semicircular Canals to Constant Speed Rotation in a Linear Acceleration Field. Washington, DC: U. S. Aeronaut, and Space Admin., 1970, SP-187, p. 353-362.

146. Steer, R. W., Y. T. Li, L. R. Young, and J. L. Meiry Physical Properties of the Labyrinthine Fluids and Quantification of the Phenomenon of Caloric Stimulation. Washington, DC: U. S. Aeronaut. and Space Admin., 1968, SP-152, p. 409-420.

147. Steinhausen, W. Über den Nachweis der Bewegung der Cupula in der intakten Bogengansampulle des Labyrinths bei der natürlichen rotatorischen und calorischen Reizung. Pfluegers Arch. Ges. Physiol. 228: 322-328, 1931.

148. Steinhausen, W. Observations of the cupula in the ampullae of the semicircular canals of a living pike. Pfluegers Arch. Ges. Physiol. 232: 500-512, 1933. (NASA TTF-13,665, 1971.)

149. Stevens, S. S. On the psychophysical law. Psychol. Rev. 64 153-181, 1957.

150. Stevens, S. S. The psychophysics of sensory function. Am. Sci. 48: 226-253, 1960 .

151. Stone, R. W., AND W. Letko. Some Observations during Weightlessness Simulated with a Subject Immersed in a Rotating Water Tank. Washington, DC: U. S. Aeronaut. and Space Admin., 1964, TND-2195.

152. Teuber, H.-L. Perception. In: Handbook of Physiology. Neu rophysiology, edited by J. Field, H. W. Magoun, and V. E. Hall. Washington, DC: Am. Physiol. Soc., 1960, sect. 1, vol. III, chapt. 65 , p. $1595-1661$.

153. Teuber, H.-L., and R. S. Liebert. Auditory vection. Am. Psychol. 11: 430, 1956.

154. Triesmann, M. Motion sickness: an evolutionary hypothesis. Science 197: 493-495, 1977.

155. Trincker, D. E. W. Neuere Aspekte der Mechanismus der Haarzell-Erregung. Acta Oto-Laryngol. Suppl. 163: 67-75, 1961.

156. Twitchell, T. E. Posture control. J. Am. Phys. Ther. Assoc. 45: 415,1965

157. Udo DE HAES, H. Stability of apparent vertical and ocular counterrolling as a function of lateral tilt. Percept. Psychophys. 8: $137-142,1970$.

158. UdO DE HAES, H., AND H. SCHÖNE. Interaction between statolith organs and semicircular canals on apparent vertical and nystagmus. Acta Oto-Laryngol. 69: 25-31, 1970.

159. Van Dishoeck, H. A. E., A. Spoor, and P. Nijhoff. The optogyral illusion and its relation to the nystagmus of the eyes.
Acta Oto-Laryngol. 44: 597-607, 1954

160. Van Egmond, A. A. J., J. J. Groen, and L. B. W. Jongkees. The mechanics of the semicircular canal. J. Physiol. London 110: 1-17, 1949.

161. Verillo, R. T. Vibrotactile thresholds for hairy skin. J. Exp. Psychol. 73: 47-50, 1966.

162. Von Holst, E., and H. Mittelstaedt. Das Reafferenzprinzip. Naturwissenschaften. 37: 464, 1950.

163. WaEspe, W., AND V. Henn. Neuronal activity in the vestibular nuclei of the alert monkey during vestibular and optokinetic stimulation. Exp. Brain Res. 27: 523-538, 1977.

164. WALSH, E. G. Role of vestibular apparatus in the perception of motion on a parallel swing. J. Physiol. London 155: 506-513, 1961.

165. WALSH, E. G. The perception of rhythmically repeated linear motion in the horizontal plane. Br. J. Psychol. 53: 439-445, 1962.

166. Wapner, S., H. Werner, And K. A. Chandler. Experiments on sensory-tonic field theory of perception: I. Effect of extraneous stimulation on the visual perception of verticality. $J$. Exp. Psychol. 42: 341-343, 1951.

166a.WeBER, E. H. Der Tastsinn und das Gemeingefühl. In: Handwörterbuch der Physiologie, edited by R. Wagner. Brunswick: Vieweg, 1846, Vol. III, pt. 2, p. 481-588. (Transl. by H. E. Ross and D. J. Murras: E. H. Weber The Sense of Touch. London: Academic, 1978.)

167. Werner, H., S. Wapner, and K. A. Chandler. Experiments on sensory-tonic field theory of perception: II. Effect of supported and unsupported tilt on the visual perception of verticality. J. Exp. Psychol. 42: 346-350, 1951.

168. Whiteside, T. D. M., A. Graybiel, and J. I. Niven. Visual illusions of movement. Brain 88: 193-210, 1965.

169. Wilson, V., ANd G. Melvill-Jones. Mammalian Vestibular Physiology. New York: Plenum, 1979.

170. Witkin, H. A. The perception of the upright. Sci. Am. 182: 50$72,1959$.

171. Witkin, H. A., AND S. E. Asch. Studies in space orientation. IV. Further experiments on perception of the upright with displaced visual fields. J. Exp. Psychol. 38: 762-778, 1948.

172. Wood, R. W. The "haunted swing" illusion. Psychol. Rev. 2: 277-278, 1895

173. Yasui, S., AND L. R. Young. Perceived visual motion as effective stimulus to pursuit eye movement system. Science 190: 906-908, 1975.

174. Young, L. R. On visual vestibular interaction. In: Proc. Fifth Symposium on the Role of the Vestibular Organs in Space Exploration. Washington, DC: U. S. Aeronaut. and Space Admin., 1970, SP-314, p. 205-210.

175. YounG, L. R. Visually induced motion in flight simulation. AGARD Conf. Proc. 249: 16-1-16-8, 1977. (Presented at the AGARD Flight Mechanics Panel Specialists' Meeting on Piloted Aircraft Environmental Simulation Techniques. Brussels, April 24-27, 1977.)

175a.Young, L. R. Man's internal navigation system. Technol. Rev. 80: 40-45, 1978.

176. Young, L. R., J. Dichgans, R. Murphy, and Th. Brandt Interaction of optokinetic and vestibular stimuli in motion perception. Acta Oto-Laryngol. 76: 24-31, 1973.

177. Young, L. R., B. K. Lichtenberg, A. P. Arrott, T. A. Crites, C. M. Oman, and E. R. Edelman. Ocular countertorsion on earth and in weightlessness. N. Y. Acad. Sci. 374: 80 92, 1981.

177a. Young, L. R., AND J. MEIRY. A revised dynamic otolith model. Aerosp. Med. 39: 606-608, 1968.

178. YounG, L. R., AND C. M. Oman. Model for vestibular adaptation to horizontal rotation. Aerosp. Med. 39: 606-608, 1969.

179. Young, L. R., C. M. OMan, and J. Dichgans. Influence of head position on visually induced pitch and roll sensation. Aviat. Space Environ. Med. 46: 264-268, 1975.

180. ZaCharias, G. L., AND L. R. Young. Influence of combined visual and vestibular cues on human perception and control of horizontal rotation. Exp. Brain Res. 41: 159-171, 1981. 